\title{
Energy Propagation in Deep Convolutional Neural Networks
}

\author{
Thomas Wiatowski, Philipp Grohs, and Helmut Bölcskei, Fellow, IEEE
}

\begin{abstract}
Many practical machine learning tasks employ very deep convolutional neural networks. Such large depths pose formidable computational challenges in training and operating the network. It is therefore important to understand how fast the energy contained in the propagated signals (a.k.a. feature maps) decays across layers. In addition, it is desirable that the feature extractor generated by the network be informative in the sense of the only signal mapping to the all-zeros feature vector being the zero input signal. This "trivial null-set" property can be accomplished by asking for "energy conservation" in the sense of the energy in the feature vector being proportional to that of the corresponding input signal. This paper establishes conditions for energy conservation (and thus for a trivial null-set) for a wide class of deep convolutional neural network-based feature extractors and characterizes corresponding feature map energy decay rates. Specifically, we consider general scattering networks employing the modulus non-linearity and we find that under mild analyticity and high-pass conditions on the filters (which encompass, inter alia, various constructions of Weyl-Heisenberg filters, wavelets, ridgelets, $(\alpha)$-curvelets, and shearlets) the feature map energy decays at least polynomially fast. For broad families of wavelets and Weyl-Heisenberg filters, the guaranteed decay rate is shown to be exponential. Moreover, we provide handy estimates of the number of layers needed to have at least $((1-\varepsilon) \cdot 100) \%$ of the input signal energy be contained in the feature vector.
\end{abstract}

Index Terms-Machine learning, deep convolutional neural networks, scattering networks, energy decay and conservation, frame theory.

\section{INTRODUCTION}

$\mathbf{F}$ EATURE extraction based on deep convolutional neural networks (DCNNs) has been applied with significant success in a wide range of practical machine learning tasks [1]-[6]. Many of these applications, such as, e.g., the classification of images in the ImageNet data set, employ very deep networks with potentially hundreds of layers [7]. Such network depths entail formidable computational challenges in the training phase due to the large number of parameters to be learned, and in operating the network due to the large number of convolutions that need to be carried out. It is therefore paramount to understand how fast the energy contained in the signals generated in the individual network

T. Wiatowski and H. Bölcskei are with the Department of Information Technology and Electrical Engineering, ETH Zurich, Switzerland. Email: \{withomas, boelcskei\}@nari.ee.ethz.ch

P. Grohs is with the Faculty of Mathematics, University of Vienna, Austria. Email: philipp.grohs@univie.ac.at

The material in this paper was presented in part at the 2017 IEEE International Symposium on Information Theory (ISIT), Aachen, Germany.

Copyright (c) 2017 IEEE. Personal use of this material is permitted. However, permission to use this material for any other purposes must be obtained from the IEEE by sending a request to pubs-permissions@ieee.org. layers, a.k.a. feature maps, decays across layers. In addition, it is important that the feature vector-obtained by aggregating filtered versions of the feature maps-be informative in the sense of the only signal mapping to the all-zeros feature vector being the zero input signal. This "trivial null-set" property for the feature extractor can be obtained by asking for the energy in the feature vector being proportional to that of the corresponding input signal, a property we shall refer to as "energy conservation".

Scattering networks as introduced in [8] and extended in [9] constitute an important class of feature extractors based on nodes that implement convolutional transforms with prespecified or learned filters in each network layer (e.g., wavelets [8], [10], uniform covering filters [11], or general filters [9]), followed by a non-linearity (e.g., the modulus [8], [10], [11], or a general Lipschitz non-linearity [9]), and a pooling operation (e.g., sub-sampling or average-pooling [9]). Scattering network-based feature extractors were shown to yield classification performance competitive with the state-of-the-art on various data sets [12]-[17]. Moreover, a mathematical theory exists, which allows to establish formally that such feature extractors are-under certain technical conditions-horizontally [8] or vertically [9] translation-invariant and deformationstable in the sense of [8], or exhibit limited sensitivity to deformations in the sense of [9] on input signal classes such as band-limited functions [9], [18], cartoon functions [19], and Lipschitz functions [19].

It was shown recently that the energy in the feature maps generated by scattering networks employing, in every network layer, the same set of (certain) Parseval wavelets [10. Section 5] or "uniform covering" [11] filters (both satisfying analyticity and vanishing moments conditions), the modulus non-linearity, and no pooling, decays at least exponentially fast and "strict" energy conservation (which, in turn, implies a trivial null-set) for the infinite-depth feature vector holds. Specifically, the feature map energy decay was shown to be at least of order $\mathcal{O}\left(a^{-N}\right)$, for some unspecified $a>1$, where $N$ denotes the network depth. We note that $d$-dimensional uniform covering filters as introduced in [11] are functions whose Fourier transforms' support sets can be covered by a union of finitely many balls. This covering condition is satisfied by, e.g., Weyl-Heisenberg filters [21] with a bandlimited prototype function, but fails to hold for multi-scale filters such as wavelets [22], [23], $(\alpha)$-curvelets [24]-[26], shearlets [27], [28], or ridgelets [29]-[31], see [11, Remark 2.2 (b)].

Contributions. The first main contribution of this paper is a characterization of the feature map energy decay rate in 
DCNNs employing the modulus non-linearity, no pooling, and general filters that constitute a frame [22], [32]-[34], but not necessarily a Parseval frame, and are allowed to be different in different network layers. We find that, under mild analyticity and high-pass conditions on the filters, the energy decay rate is at least polynomial in the network depth, i.e., the decay is at least of order $\mathcal{O}\left(N^{-\alpha}\right)$, and we explicitly specify the decay exponent $\alpha>0$. This result encompasses, inter alia, various constructions of Weyl-Heisenberg filters, wavelets, ridgelets, $(\alpha)$-curvelets, shearlets, and learned filters (of course as long as the learning algorithm imposes the analyticity and high-pass conditions we require). For broad families of wavelets and Weyl-Heisenberg filters, the guaranteed energy decay rate is shown to be exponential in the network depth, i.e., the decay is at least of order $\mathcal{O}\left(a^{-N}\right)$ with the decay factor given as $a=\frac{5}{3}$ in the wavelet case and $a=\frac{3}{2}$ in the Weyl-Heisenberg case. We hasten to add that our results constitute guaranteed decay rates and do not preclude the energy from decaying faster in practice.

Our second main contribution shows that the energy decay results above are compatible with a trivial null-set for finiteand infinite-depth networks. Specifically, this is accomplished by establishing energy proportionality between the feature vector and the underlying input signal with the proportionality constant lower- and upper-bounded by the frame bounds of the filters employed in the different layers. We show that this energy conservation result is a consequence of a demodulation effect induced by the modulus non-linearity in combination with the analyticity and high-pass properties of the filters. Specifically, in every network layer, the modulus non-linearity moves the spectral content of each individual feature map to base-band (i.e., to low frequencies), where it is subsequently extracted (i.e., fed into the feature vector) by a low-pass output-generating filter.

Finally, for input signals that belong to the class of Sobolev functions 1 our energy decay and conservation results are shown to yield handy estimates of the number of layers needed to have at least $((1-\varepsilon) \cdot 100) \%$ of the input signal energy be contained in the feature vector. For example, in the case of exponential energy decay with $a=\frac{5}{3}$ and for band-limited input signals, only 8 layers are needed to absorb $95 \%$ of the input signal's energy.

We emphasize that throughout energy decay results pertain to the feature maps, whereas energy conservation statements apply to the feature vector, obtained by aggregating filtered versions of the feature maps.

Notation. The complex conjugate of $z \in \mathbb{C}$ is denoted by $\bar{z}$. We write $\operatorname{Re}(z)$ for the real, and $\operatorname{Im}(z)$ for the imaginary part of $z \in \mathbb{C}$. The Euclidean inner product of $x, y \in \mathbb{C}^{d}$ is $\langle x, y\rangle:=\sum_{i=1}^{d} x_{i} \overline{y_{i}}$, with associated norm $|x|:=\sqrt{\langle x, x\rangle}$. For $x \in \mathbb{R},(x)_{+}:=\max \{0, x\}$ and $\langle x\rangle:=\left(1+|x|^{2}\right)^{1 / 2}$. We denote the open ball of radius $r>0$ centered at $x \in \mathbb{R}^{d}$ by $B_{r}(x) \subseteq \mathbb{R}^{d}$. The first canonical orthant is $H:=\{x \in$

\footnotetext{
${ }^{1}$ A wide range of practically relevant signal classes are Sobolev functions, for example, band-limited functions and-as established in the present paper - cartoon functions [35]. We note that cartoon functions are widely used in the mathematical signal processing literature [15], [19], [26], [36], [37] as a model for natural images such as, e.g., images of handwritten digits [38].
}

$\left.\mathbb{R}^{d} \mid x_{k} \geq 0, k=1, \ldots, d\right\}$, and we define the rotated orthant $H_{A}:=\{A x \mid x \in H\}$ for $A \in O(d)$, where $O(d)$ stands for the orthogonal group of dimension $d \in \mathbb{N}$. The Minkowski sum of sets $A, B \subseteq \mathbb{R}^{d}$ is $(A+B):=\{a+b \mid a \in A, b \in B\}$, and $A \Delta B:=(A \backslash B) \cup(B \backslash A)$ denotes their symmetric difference. A multi-index $\alpha=\left(\alpha_{1}, \ldots, \alpha_{d}\right) \in \mathbb{N}_{0}^{d}$ is an ordered $d$-tuple of non-negative integers $\alpha_{i} \in \mathbb{N}_{0}$.

For functions $W: \mathbb{N} \rightarrow \mathbb{R}$ and $G: \mathbb{N} \rightarrow \mathbb{R}$, we say that $W(N)=\mathcal{O}(G(N))$ if there exist $C>0$ and $N_{0} \in \mathbb{N}$ such that $W(N) \leq C G(N)$, for all $N \geq N_{0}$. The support $\operatorname{supp}(f)$ of a function $f: \mathbb{R}^{d} \rightarrow \mathbb{C}$ is the closure of the set $\left\{x \in \mathbb{R}^{d} \mid f(x) \neq 0\right\}$ in the topology induced by the Euclidean norm $|\cdot|$. For a Lebesgue-measurable function $f: \mathbb{R}^{d} \rightarrow \mathbb{C}$, we write $\int_{\mathbb{R}^{d}} f(x) \mathrm{d} x$ for its integral w.r.t. Lebesgue measure. The indicator function of a set $B \subseteq \mathbb{R}^{d}$ is defined as $\mathbb{1}_{B}(x)=1$, for $x \in B$, and $\mathbb{1}_{B}(x)=0$, for $x \in \mathbb{R}^{d} \backslash B$. For a measurable set $B \subseteq \mathbb{R}^{d}$, we let $\operatorname{vol}^{d}(B):=\int_{\mathbb{R}^{d}} \mathbb{1}_{B}(x) \mathrm{d} x=\int_{B} 1 \mathrm{~d} x$, and we write $\partial B$ for its boundary. $L^{p}\left(\mathbb{R}^{d}\right)$, with $p \in[1, \infty)$, stands for the space of Lebesgue-measurable functions $f: \mathbb{R}^{d} \rightarrow \mathbb{C}$ satisfying $\|f\|_{p}:=\left(\int_{\mathbb{R}^{d}}|f(x)|^{p} \mathrm{~d} x\right)^{1 / p}<\infty . L^{\infty}\left(\mathbb{R}^{d}\right)$ denotes the space of Lebesgue-measurable functions $f: \mathbb{R}^{d} \rightarrow \mathbb{C}$ such that $\|f\|_{\infty}:=\inf \left\{\alpha>0|| f(x) \mid \leq \alpha\right.$ for a.e $\left.{ }^{2} x \in \mathbb{R}^{d}\right\}<\infty$. For a countable set $\mathcal{Q},\left(L^{2}\left(\mathbb{R}^{d}\right)\right)^{\mathcal{Q}}$ stands for the space of sets $S:=\left\{f_{q}\right\}_{q \in \mathcal{Q}}$, with $f_{q} \in L^{2}\left(\mathbb{R}^{d}\right)$ for all $q \in \mathcal{Q}$, satisfying $\||S|\|:=\left(\sum_{q \in \mathcal{Q}}\left\|f_{q}\right\|_{2}^{2}\right)^{1 / 2}<\infty$. We denote the Fourier transform of $f \in L^{1}\left(\mathbb{R}^{d}\right)$ by $\widehat{f}(\omega):=\int_{\mathbb{R}^{d}} f(x) e^{-2 \pi i\langle x, \omega\rangle} \mathrm{d} x$ and extend it in the usual way to $L^{2}\left(\mathbb{R}^{d}\right)$ [40, Theorem 7.9].

Id : $L^{p}\left(\mathbb{R}^{d}\right) \rightarrow L^{p}\left(\mathbb{R}^{d}\right)$ stands for the identity operator on $L^{p}\left(\mathbb{R}^{d}\right)$. The convolution of $f \in L^{2}\left(\mathbb{R}^{d}\right)$ and $g \in L^{1}\left(\mathbb{R}^{d}\right)$ is $(f * g)(y):=\int_{\mathbb{R}^{d}} f(x) g(y-x) \mathrm{d} x$. We write $\left(T_{t} f\right)(x):=$ $f(x-t), t \in \mathbb{R}^{d}$, for the translation operator, and $\left(M_{\omega} f\right)(x):=$ $e^{2 \pi i\langle x, \omega\rangle} f(x), \omega \in \mathbb{R}^{d}$, for the modulation operator. We set $\langle f, g\rangle:=\int_{\mathbb{R}^{d}} f(x) \overline{g(x)} \mathrm{d} x$, for $f, g \in L^{2}\left(\mathbb{R}^{d}\right)$.

$H^{s}\left(\mathbb{R}^{d}\right)$, with $s>0$, stands for the Sobolev space of functions $f \in L^{2}\left(\mathbb{R}^{d}\right)$ satisfying $\|f\|_{H^{s}}:=\left(\int_{\mathbb{R}^{d}}|\widehat{f}(\omega)|^{2}(1+\right.$ $\left.\left.|\omega|^{2}\right)^{s} \mathrm{~d} \omega\right)^{1 / 2}<\infty$, see [41. Section 6.2.1]. Here, the index $s$ reflects the degree of smoothness of $f \in H^{s}\left(\mathbb{R}^{d}\right)$, i.e., larger $s$ entails smoother $f$. For a multi-index $\alpha \in \mathbb{N}_{0}^{d}, D^{\alpha}$ denotes the differential operator $D^{\alpha}:=\left(\partial / \partial x_{1}\right)^{\alpha_{1}} \ldots\left(\partial / \partial x_{d}\right)^{\alpha_{d}}$, with order $|\alpha|:=\sum_{i=1}^{d} \alpha_{i}$. The space of functions $f: \mathbb{R}^{d} \rightarrow \mathbb{C}$ whose derivatives $D^{\alpha} f$ of order at most $k \in \mathbb{N}_{0}$ are continuous is designated by $C^{k}\left(\mathbb{R}^{d}, \mathbb{C}\right)$. Moreover, we denote the gradient of a function $f: \mathbb{R}^{d} \rightarrow \mathbb{C}$ as $\nabla f$.

\section{DCNN-BASED FEATURE EXTRACTORS}

Throughout the paper, we use the terminology of [9], consider (unless explicitly stated otherwise) input signals $f \in L^{2}\left(\mathbb{R}^{d}\right)$, and employ the module-sequence

$$
\Omega:=\left(\left(\Psi_{n},|\cdot|, \mathrm{Id}\right)\right)_{n \in \mathbb{N}},
$$

i.e., each network layer is associated with (i) a collection of filters $\Psi_{n}:=\left\{\chi_{n}\right\} \cup\left\{g_{\lambda_{n}}\right\}_{\lambda_{n} \in \Lambda_{n}} \subseteq L^{1}\left(\mathbb{R}^{d}\right) \cap L^{2}\left(\mathbb{R}^{d}\right)$, where $\chi_{n}$, referred to as output-generating filter, and the $g_{\lambda_{n}}$, indexed

\footnotetext{
${ }^{2}$ Throughout the paper "a.e." is w.r.t. Lebesgue measure.
} 


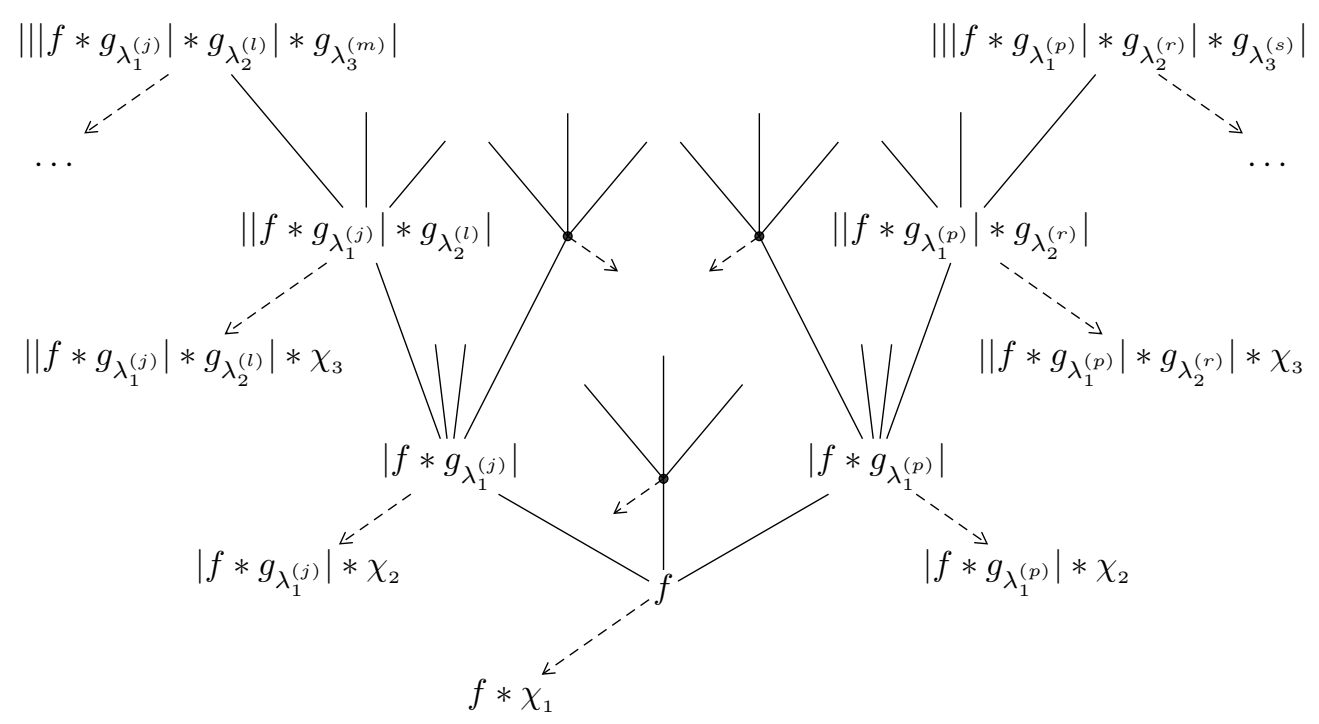

Fig. 1: Network architecture underlying the feature extractor 5. The index $\lambda_{n}^{(k)}$ corresponds to the $k$-th filter $g_{\lambda_{n}}^{(k)}$ of the collection $\Psi_{n}$ associated with the $n$-th network layer. The function $\chi_{n+1}$ is the output-generating filter of the $n$-th network layer. The root of the network corresponds to $n=0$.

by a countable set $\Lambda_{n}$, satisfy the frame condition [22], [32], [34]

$$
A_{n}\|f\|_{2}^{2} \leq\left\|f * \chi_{n}\right\|_{2}^{2}+\sum_{\lambda_{n} \in \Lambda_{n}}\left\|f * g_{\lambda_{n}}\right\|^{2} \leq B_{n}\|f\|_{2}^{2}
$$

for all $f \in L^{2}\left(\mathbb{R}^{d}\right)$, for some $A_{n}, B_{n}>0$, (ii) the modulus non-linearity $|\cdot|: L^{2}\left(\mathbb{R}^{d}\right) \rightarrow L^{2}\left(\mathbb{R}^{d}\right),|f|(x):=|f(x)|$, and (iii) no pooling, which, in the terminology of [9], corresponds to pooling through the identity operator with pooling factor equal to one. Associated with the module $\left(\Psi_{n},|\cdot|, \mathrm{Id}\right)$, the operator $U_{n}\left[\lambda_{n}\right]$ defined in [9. Eq. 12] particularizes to

$$
U_{n}\left[\lambda_{n}\right] f=\left|f * g_{\lambda_{n}}\right| .
$$

We extend (3) to paths on index sets $q=\left(\lambda_{1}, \lambda_{2}, \ldots, \lambda_{n}\right) \in \Lambda_{1} \times \Lambda_{2} \times \cdots \times \Lambda_{n}=: \Lambda^{n}, \quad n \in \mathbb{N}$, according to

$$
\begin{aligned}
U[q] f & =U\left[\left(\lambda_{1}, \lambda_{2}, \ldots, \lambda_{n}\right)\right] f \\
& :=U_{n}\left[\lambda_{n}\right] \cdots U_{2}\left[\lambda_{2}\right] U_{1}\left[\lambda_{1}\right] f,
\end{aligned}
$$

where, for the empty path $e:=\emptyset$, we set $\Lambda^{0}:=\{e\}$ and $U[e] f:=f$. The signals $U[q] f, q \in \Lambda^{n}$, associated with the $n$-th network layer, are often referred to as feature maps in the deep learning literature. The feature vector $\Phi_{\Omega}(f)$ is obtained by aggregating filtered versions of the feature maps. More formally, $\Phi_{\Omega}(f)$ is defined as [9. Definition 3]

$$
\Phi_{\Omega}(f):=\bigcup_{n=0}^{\infty} \Phi_{\Omega}^{n}(f),
$$

where

$$
\Phi_{\Omega}^{n}(f):=\left\{(U[q] f) * \chi_{n+1}\right\}_{q \in \Lambda^{n}}
$$

are the features generated in the $n$-th network layer, see Figure 1 Here, $n=0$ corresponds to the root of the network. The function $\chi_{n+1}$ is the output-generating filter of the $n$-th network layer. The feature extractor

$$
\Phi_{\Omega}: L^{2}\left(\mathbb{R}^{d}\right) \rightarrow\left(L^{2}\left(\mathbb{R}^{d}\right)\right)^{\bigcup_{n=0}^{\infty} \Lambda^{n}}
$$

was shown in [9. Theorem 1] to be vertically translationinvariant, provided although that pooling is employed, with pooling factors $S_{n} \geq 1, n \in \mathbb{N}$, (see [9. Eq. 6] for the definition of the general pooling operator) such that $\lim _{N \rightarrow \infty} \prod_{n=1}^{N} S_{n}=\infty$. Moreover, $\Phi_{\Omega}$ exhibits limited sensitivity to certain non-linear deformations on (input) signal classes such as band-limited functions [9. Theorem 2], cartoon functions [19, Theorem 1], and Lipschitz functions [19. Corollary $1]$.

\section{ENERGY DECAY AND TRIVIAL NULL-SET}

The first central goal of this paper is to understand how fast the energy contained in the feature maps decays across layers. Specifically, we shall study the decay of

$$
W_{N}(f):=\sum_{q \in \Lambda^{N}}\|U[q] f\|_{2}^{2}, \quad f \in L^{2}\left(\mathbb{R}^{d}\right),
$$

as a function of network depth $N$. Moreover, it is desirable that the infinite-depth feature vector $\Phi_{\Omega}(f)$ be informative in the sense of the only signal mapping to the all-zeros feature vector being the zero input signal, i.e., $\Phi_{\Omega}$ has a trivial null-set

$$
\mathcal{N}\left(\Phi_{\Omega}\right):=\left\{f \in L^{2}\left(\mathbb{R}^{d}\right) \mid \Phi_{\Omega}(f)=0\right\} \stackrel{!}{=}\{0\} .
$$

Figure 2 illustrates the practical ramifications of a non-trivial null-set in a binary classification task. $\mathcal{N}\left(\Phi_{\Omega}\right)=\{0\}$ can be guaranteed by asking for "energy conservation" in the sense of

$$
A_{\Omega}\|f\|_{2}^{2} \leq\left\||| \Phi_{\Omega}(f)\right\|\left\|^{2} \leq B_{\Omega}\right\| f \|_{2}^{2}, \quad \forall f \in L^{2}\left(\mathbb{R}^{d}\right),
$$

for some constants $A_{\Omega}, B_{\Omega}>0$ (possibly depending on the module-sequence $\Omega$ ) and with the feature space norm $\left\||| \Phi_{\Omega}(f) \mid\right\|:=\left(\left.\sum_{n=0}^{\infty}||\left|\Phi_{\Omega}^{n}(f)\right|\right|^{2}\right)^{1 / 2}$, where $\left\|\left|\Phi_{\Omega}^{n}(f)\right|\right\|:=$ 


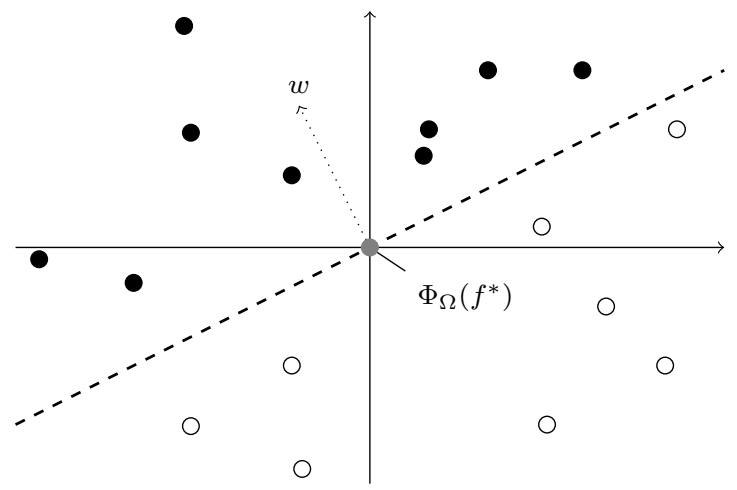

Fig. 2: Impact of a non-trivial null-set $\mathcal{N}\left(\Phi_{\Omega}\right)$ in a binary classification task. The feature vector $\Phi_{\Omega}(f)$ is fed into a linear classifier [20], which determines set membership based on the sign of the inner product $\left\langle w, \Phi_{\Omega}(f)\right\rangle$. The (learned) weight vector $w$ is perpendicular to the separating hyperplane (dashed line). If the null-set of the feature extractor $\Phi_{\Omega}$ is non-trivial, there exist input signals $f^{*} \neq 0$ that are mapped to the origin in feature space, i.e., $\Phi_{\Omega}\left(f^{*}\right)=0$ (gray circle), and therefore lie-independently of the weight vector $w$-on the separating hyperplane. These input signals $f^{*} \neq 0$ are therefore unclassifiable.

$\left(\sum_{q \in \Lambda^{n}}\left\|(U[q] f) * \chi_{n+1}\right\|_{2}^{2}\right)^{1 / 2}$. Indeed, (7) follows from (8) as the upper bound in $(8)$ yields $\{0\} \subseteq \mathcal{N}\left(\Phi_{\Omega}\right)$, and the lower bound implies $\{0\} \supseteq \mathcal{N}\left(\Phi_{\Omega}\right)$. We emphasize that, as $\Phi_{\Omega}$ is a non-linear operator (owing to the modulus non-linearities), characterizing its null-set is non-trivial in general. The upper bound in (8) was established in [9. Appendix E]. While the existence of this upper bound is implied by the filters $\Psi_{n}$, $n \in \mathbb{N}$, satisfying the frame property (2) [9. Appendix E], perhaps surprisingly, this is not enough to guarantee $A_{\Omega}>0$ (see Appendix A for an example). We refer the reader to Section $\mathrm{V}$ for results on the null-set of the finite-depth feature extractor $\bigcup_{n=0}^{N} \Phi_{\Omega}^{n}$.

Previous work on the decay rate of $W_{N}(f)$ in [10, Section 5] shows that for wavelet-based networks (i.e., in every network layer the filters $\Psi=\{\chi\} \cup\left\{g_{\lambda}\right\}_{\lambda \in \Lambda}$ in (1) are taken to be (specific) 1-D wavelets that constitute a Parseval frame, with $\chi$ a low-pass filter) there exist $\varepsilon>0$ and $a>1$ (both constants unspecified) such that

$$
W_{N}(f) \leq \int_{\mathbb{R}}|\widehat{f}(\omega)|^{2}\left(1-\left|\widehat{r_{g}}\left(\frac{\omega}{\varepsilon a^{N-1}}\right)\right|^{2}\right) \mathrm{d} \omega,
$$

for real-valued 1-D signals $f \in L^{2}(\mathbb{R})$ and $N \geq 2$, where $\widehat{r_{g}}(\omega):=e^{-\omega^{2}}$. To see that this result indicates energy decay, Figure 3 illustrates the influence of network depth $N$ on the upper bound in (9). Specifically, we can see that increasing the network depth results in cutting out increasing amounts of energy of $f$ and thereby making the upper bound in 9 decay as a function of $N$. Moreover, it is interesting to note that the upper bound on $W_{N}(f)=\sum_{q \in \Lambda^{N}}\|U[q] f\|_{2}^{2}$ is independent of the wavelets generating the feature maps $U[q] f, q \in \Lambda^{N}$. For scattering networks that employ, in every network layer, uniform covering filters $\Psi=\{\chi\} \cup\left\{g_{\lambda}\right\}_{\lambda \in \Lambda} \subseteq L^{1}\left(\mathbb{R}^{d}\right) \cap$ $L^{2}\left(\mathbb{R}^{d}\right)$ forming a Parseval frame (where $\chi$, again, is a lowpass filter), exponential energy decay according to

$$
W_{N}(f)=\mathcal{O}\left(a^{-N}\right), \quad \forall f \in L^{2}\left(\mathbb{R}^{d}\right),
$$

for an unspecified $a>1$, was established in [11, Proposition 3.3]. Moreover, [10, Section 5] and [11, Theorem 3.6 (a)] state-for the respective module-sequences-that 8 holds with $A_{\Omega}=B_{\Omega}=1$ and hence

$$
\left\|\mid \Phi_{\Omega}(f)\right\|^{2}=\|f\|_{2}^{2} .
$$

The first main goal of the present paper is to establish i) for $d$ dimensional complex-valued input signals that $W_{N}(f)$ decays polynomially according to

$$
W_{N}(f) \leq B_{\Omega}^{N} \int_{\mathbb{R}^{d}}|\widehat{f}(\omega)|^{2}\left(1-\left|\widehat{r}_{l}\left(\frac{\omega}{N^{\alpha}}\right)\right|^{2}\right) \mathrm{d} \omega,
$$

for $f \in L^{2}\left(\mathbb{R}^{d}\right)$ and $N \geq 1$, where $\alpha=1$, for $d=1$, and $\alpha=$ $\log _{2}(\sqrt{d /(d-1 / 2)})$, for $d \geq 2, B_{\Omega}^{N}=\prod_{k=1}^{N} \max \left\{1, B_{k}\right\}$, and $\widehat{r}_{l}: \mathbb{R}^{d} \rightarrow \mathbb{R}, \widehat{r}_{l}(\omega)=(1-|\omega|)_{+}^{l}$, with $l>\lfloor d / 2\rfloor+$ 1 , for networks based on general filters $\left\{\chi_{n}\right\} \cup\left\{g_{\lambda_{n}}\right\}_{\lambda_{n} \in \Lambda_{n}}$ that satisfy mild analyticity and high-pass conditions and are allowed to be different in different network layers (with the proviso that $\chi_{n}, n \in \mathbb{N}$, is of low-pass nature in a sense to be made precise), and ii) for 1-D complex-valued input signals that (6) decays exponentially according to

$$
W_{N}(f) \leq \int_{\mathbb{R}}|\widehat{f}(\omega)|^{2}\left(1-\left|\widehat{r}_{l}\left(\frac{\omega}{a^{N-1}}\right)\right|^{2}\right) \mathrm{d} \omega,
$$

for $f \in L^{2}(\mathbb{R})$ and $N \geq 1$, for networks that are based, in every network layer, on a broad family of wavelets, with the decay factor given explicitly as $a=\frac{5}{3}$, or on a broad family of Weyl-Heisenberg filters [9. Appendix B], with decay factor $a=\frac{3}{2}$. Thanks to the right-hand side (RHS) of (12) and (13) not depending on the specific filters $\left\{\chi_{n}\right\} \cup\left\{g_{\lambda_{n}}\right\}_{\lambda_{n} \in \Lambda_{n}}$, we will be able to establish-under smoothness assumptions on the input signal $f$-universal energy decay results. Specifically, particularizing the RHS expressions in (12) and (13) to Sobolev-class input signals $f \in H^{s}\left(\mathbb{R}^{d}\right), s>0$, where

$$
H^{s}\left(\mathbb{R}^{d}\right)=\left\{f \in L^{2}\left(\mathbb{R}^{d}\right) \mid\|f\|_{H^{s}}<\infty\right\},
$$

with $\|f\|_{H^{s}}:=\left(\int_{\mathbb{R}^{d}}\left(1+|\omega|^{2}\right)^{s}|\widehat{f}(\omega)|^{2} \mathrm{~d} \omega\right)^{1 / 2}$, we show that (12) yields polynomial energy decay according to

$$
W_{N}(f)=\mathcal{O}\left(N^{-\gamma \alpha}\right),
$$

and (13) exponential energy decay

$$
W_{N}(f)=\mathcal{O}\left(a^{-\gamma N}\right),
$$

where $\gamma:=\min \{1,2 s\}$ in both cases. Sobolev spaces $H^{s}\left(\mathbb{R}^{d}\right)$ contain a wide range of practically relevant signal classes such as, e.g.,

- the space $L_{L}^{2}\left(\mathbb{R}^{d}\right):=\left\{f \in L^{2}\left(\mathbb{R}^{d}\right) \mid \operatorname{supp}(\widehat{f}) \subseteq\right.$ $\left.B_{L}(0)\right\}, L \geq 0$, of $L$-band-limited functions according to $L_{L}^{2}\left(\mathbb{R}^{d}\right) \subseteq H^{s}\left(\mathbb{R}^{d}\right)$, for $L \geq 0$ and $s>0$. This follows from

$$
\begin{aligned}
\int_{\mathbb{R}^{d}}\left(1+|\omega|^{2}\right)^{s}|\widehat{f}(\omega)|^{2} \mathrm{~d} \omega & =\int_{B_{L}(0)}\left(1+|\omega|^{2}\right)^{s}|\widehat{f}(\omega)|^{2} \mathrm{~d} \omega \\
& \leq\left(1+|L|^{2}\right)^{s}\|f\|_{2}^{2}<\infty,
\end{aligned}
$$

for $f \in L_{L}^{2}\left(\mathbb{R}^{d}\right), L \geq 0$, and $s>0$, where we used Parseval's formula and the fact that $\omega \mapsto\left(1+|\omega|^{2}\right)^{s}$, 

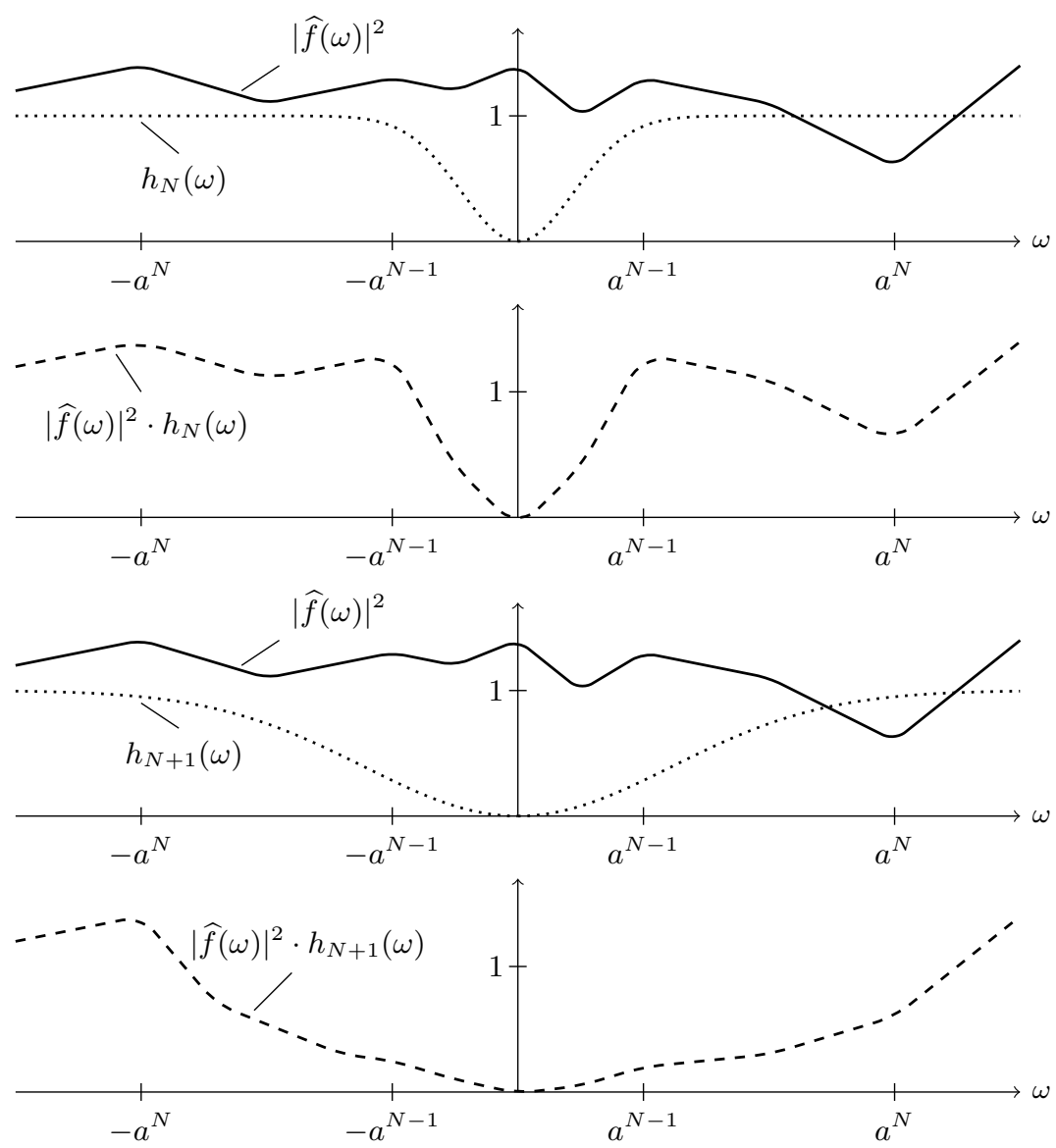

Fig. 3: Illustration of the impact of network depth $N$ on the upper bound on $W_{N}(f)$ in 9 , for $\varepsilon=1$ and $a>1$. The function $h_{N}(\omega):=\left(1-\widehat{r_{g}}\left(\frac{\omega}{\varepsilon a^{N-1}}\right)\right)$, where $\widehat{r_{g}}(\omega)=e^{-\omega^{2}}$, is of increasing high-pass nature as $N$ increases, which makes the upper bound in 9 decay in $N$.

$\omega \in \mathbb{R}^{d}$, is monotonically increasing in $|\omega|$, for $s>0$,

- the space $\mathcal{C}_{\mathrm{CART}}^{K}$ of cartoon functions of size $K$, introduced in [35], and widely used in the mathematical signal processing literature [15], [19], [26], [36], [37] as a model for natural images such as, e.g., images of handwritten digits [38] (see Figure 44). For a formal definition of $\mathcal{C}_{\mathrm{CART}}^{K}$, we refer the reader to Appendix $\mathrm{B}$, where we also show that $\mathcal{C}_{\mathrm{CART}}^{K} \subseteq H^{s}\left(\mathbb{R}^{d}\right)$, for $K>0$ and $s \in(0,1 / 2)$.

Moreover, Sobolev functions are contained in the space of $k$-times continuously differentiable functions $C^{k}\left(\mathbb{R}^{d}, \mathbb{C}\right)$ according to $H^{s}\left(\mathbb{R}^{d}\right) \subseteq C^{k}\left(\mathbb{R}^{d}, \mathbb{C}\right)$, for $s>k+\frac{d}{2}[42$, Section 4].

Our second central goal is to prove energy conservation according to 8 ( 8 (which, as explained above, implies $\mathcal{N}\left(\Phi_{\Omega}\right)=$ $\{0\})$ for the network configurations corresponding to the energy decay results (12) and (13). Finally, we provide handy estimates of the number of layers needed to have at least $((1-\varepsilon) \cdot 100) \%$ of the input signal energy be contained in the feature vector.

\section{MAin Results}

Throughout the paper, we make the following assumptions on the filters $\left\{g_{\lambda_{n}}\right\}_{\lambda_{n} \in \Lambda_{n}}$.
Assumption 1. The $\left\{g_{\lambda_{n}}\right\}_{\lambda_{n} \in \Lambda_{n}}, n \in \mathbb{N}$, are analytic in the following sense: For every layer index $n \in \mathbb{N}$, for every $\lambda_{n} \in$ $\Lambda_{n}$, there exists an orthant $H_{A_{\lambda_{n}}} \subseteq \mathbb{R}^{d}$, with $A_{\lambda_{n}} \in O(d)$, such that

$$
\operatorname{supp}\left(\widehat{g_{\lambda_{n}}}\right) \subseteq H_{A_{\lambda_{n}}} .
$$

Moreover, there exists $\delta>0$ so that

$$
\sum_{\lambda_{n} \in \Lambda_{n}}\left|\widehat{g_{\lambda_{n}}}(\omega)\right|^{2}=0, \quad \text { a.e. } \omega \in B_{\delta}(0) \text {. }
$$

In the 1-D case, i.e., for $d=1$, Assumption 1 simply amounts to every filter $g_{\lambda_{n}}$ satisfying either

$$
\operatorname{supp}\left(\widehat{g_{\lambda_{n}}}\right) \subseteq(-\infty,-\delta] \quad \text { or } \operatorname{supp}\left(\widehat{g_{\lambda_{n}}}\right) \subseteq[\delta, \infty),
$$

which constitutes an "analyticity" and "high-pass" condition. For dimensions $d \geq 2$, Assumption 1 requires that every filter $g_{\lambda_{n}}$ be of high-pass nature and have a Fourier transform supported in a (not necessarily canonical) orthant. Since the frame condition (2) is equivalent to the Littlewood-Paley condition [43]

$$
A_{n} \leq\left|\widehat{\chi_{n}}(\omega)\right|^{2}+\sum_{\lambda_{n} \in \Lambda_{n}}\left|\widehat{g_{\lambda_{n}}}(\omega)\right|^{2} \leq B_{n}, \quad \text { a.e. } \omega \in \mathbb{R}^{d},
$$

(17) implies low-pass characteristics for $\chi_{n}$ to fill the spectral gap $B_{\delta}(0)$ left by the filters $\left\{g_{\lambda_{n}}\right\}_{\lambda_{n} \in \Lambda_{n}}$. 


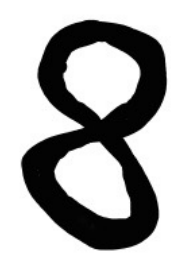

Fig. 4: An image of a handwritten digit is modeled by a 2-D cartoon function.

The conditions (16) and 17) we impose on the $\Psi_{n}, n \in \mathbb{N}$, are not overly restrictive as they encompass, inter alia, various constructions of Weyl-Heisenberg filters (e.g., a 1-D $B$-spline as prototype function [45, Section 1]), wavelets (e.g., analytic Meyer wavelets [22, Section 3.3.5] in 1-D, and Cauchy wavelets [46] in 2-D), and specific constructions of ridgelets [31, Section 2.2], curvelets [25, Section 4.1], $\alpha$-curvelets [26. Section 3], and shearlets (e.g., cone-adapted shearlets [37. Section 4.3]). We refer the reader to [9. Appendices B and C] for a brief review of some of these filter structures.

We are now ready to state our main result on energy decay and energy conservation.

Theorem 1. Let $\Omega$ be the module-sequence (1) with filters $\left\{g_{\lambda_{n}}\right\}_{\lambda_{n} \in \Lambda_{n}}$ satisfying the conditions in Assumption 1] and let $\delta>0$ be the radius of the spectral gap $B_{\delta}(0)$ left by the filters $\left\{g_{\lambda_{n}}\right\}_{\lambda_{n} \in \Lambda_{n}}$ according to (17). Furthermore, let $s \geq 0$, $A_{\Omega}^{N}:=\prod_{k=1}^{N} \min \left\{1, A_{k}\right\}, B_{\Omega}^{N}:=\prod_{k=1}^{N} \max \left\{1, B_{k}\right\}$, and

$$
\alpha:= \begin{cases}1, & d=1, \\ \log _{2}(\sqrt{d /(d-1 / 2)}), & d \geq 2 .\end{cases}
$$

i) We have

$$
W_{N}(f) \leq B_{\Omega}^{N} \int_{\mathbb{R}^{d}}|\widehat{f}(\omega)|^{2}\left(1-\left|\widehat{r_{l}}\left(\frac{\omega}{N^{\alpha} \delta}\right)\right|^{2}\right) \mathrm{d} \omega,
$$

for $f \in L^{2}\left(\mathbb{R}^{d}\right)$ and $N \geq 1$, where $\widehat{r_{l}}: \mathbb{R}^{d} \rightarrow \mathbb{R}$, $\widehat{r_{l}}(\omega):=(1-|\omega|)_{+}^{l}$, with $l>\lfloor d / 2\rfloor+1$.

ii) For every Sobolev function $f \in H^{s}\left(\mathbb{R}^{d}\right), s>0$, we have

$$
W_{N}(f)=\mathcal{O}\left(B_{\Omega}^{N} N^{-\gamma \alpha}\right),
$$

where $\gamma:=\min \{1,2 s\}$.

iii) If, in addition to Assumption 1 ,

$$
0<A_{\Omega}:=\lim _{N \rightarrow \infty} A_{\Omega}^{N} \leq B_{\Omega}:=\lim _{N \rightarrow \infty} B_{\Omega}^{N}<\infty,
$$

then we have energy conservation according to

$$
A_{\Omega}\|f\|_{2}^{2} \leq\|\| \Phi_{\Omega}(f)\left\|^{2} \leq B_{\Omega}\right\| f \|_{2}^{2},
$$

for all $f \in L^{2}\left(\mathbb{R}^{d}\right)$.

Proof. For the proofs of i) and ii), we refer to Appendices C and $\mathrm{D}$, respectively. The proof of statement iii) is based on two key ingredients. First, we establish - in Proposition 1 in Appendix $\mathrm{E}-$ that the feature extractor $\Phi_{\Omega}$ satisfies the energy decomposition identity

$$
A_{\Omega}^{N}\|f\|_{2}^{2} \leq \sum_{n=0}^{N-1}\|\| \Phi_{\Omega}^{n}(f)\|\|^{2}+W_{N}(f) \leq B_{\Omega}^{N}\|f\|_{2}^{2},
$$

for all $f \in L^{2}\left(\mathbb{R}^{d}\right)$ and all $N \geq 1$. Second, we show-in Proposition 2 in Appendix $\mathrm{F}$-that the integral on the RHS of 20] goes to zero as $N \rightarrow \infty$ which, thanks to $\lim _{N \rightarrow \infty} B_{\Omega}^{N}=$ $B_{\Omega}<\infty$, implies that $W_{N}(f) \rightarrow 0$ as $N \rightarrow \infty$. We note that while the decomposition (24) holds for general filters $\Psi_{n}$ satisfying the frame property (2), it is the upper bound (20) that makes use of the analyticity and high-pass conditions in Assumption 1. The final energy conservation result 23] is obtained by letting $N \rightarrow \infty$ in (24).

The strength of the results in Theorem 1 derives itself from the fact that the only condition we need to impose on the filters $\Psi_{n}$ is Assumption 11. which, as already mentioned, is met by a wide array of filters. Moreover, condition (22) is easily satisfied by normalizing the filters $\Psi_{n}, n \in \mathbb{N}$, appropriately (see, e.g., [9, Proposition 3]). We note that this normalization, when applied to filters that satisfy Assumption 1 . yields filters that still meet Assumption 1 .

The identity (21) establishes, upon normalization [9, Proposition 3] of the $\Psi_{n}$ to get $B_{n} \leq 1, n \in \mathbb{N}$, that the energy decay rate, i.e., the decay rate of $W_{N}(f)$, is at least polynomial in $N$. We hasten to add that 20 does not preclude the energy from decaying faster in practice.

Underlying the energy conservation result (23) is the following demodulation effect induced by the modulus nonlinearity in combination with the analyticity and high-pass properties of the filters $\left\{g_{\lambda_{n}}\right\}_{\lambda_{n} \in \Lambda_{n}}$. In every network layer, the spectral content of each individual feature map is moved to base-band (i.e., to low frequencies), where it is extracted by the low-pass output-generating atom $\chi_{n+1}$, see Figure 5 . The components not collected by $\chi_{n+1}$ (see Figure 5 . bottom row) are captured by the analytic high-pass filters $\left\{g_{\lambda_{n+1}}\right\}_{\lambda_{n+1} \in \Lambda_{n+1}}$ in the next layer and, thanks to the modulus non-linearity, again moved to low frequencies and extracted by $\chi_{n+2}$. Iterating this process ensures that the null-set of the feature vector (be it for the infinite-depth network or, as established in Section $\mathrm{V}$, for finite network depths) is trivial. It is interesting to observe that the sigmoid, the rectified linear unit, and the hyperbolic tangent non-linearities-all widely used in the deep learning literature-exhibit very different behavior in this regard, namely, they do not demodulate in the way the modulus non-linearity does [44. Figure 6]. It is therefore unclear whether the proof machinery for energy conservation developed in this paper extends to these nonlinearities or, for that matter, whether one gets energy decay and conservation at all.

The feature map energy decay result (21) relates to the feature vector energy conservation result 23 via the energy decomposition identity (24). Specifically, particularizing (24) for Parseval frames, i.e., $A_{n}=B_{n}=1$, for all $n \in \mathbb{N}$, we get

$$
\sum_{n=0}^{N-1}\|\| \Phi_{\Omega}^{n}(f)\|\|^{2}+W_{N}(f)=\|f\|_{2}^{2} .
$$

This shows that the input signal energy contained in the network layers $n \geq N$ is precisely given by $W_{N}(f)$. Thanks to $W_{N}(f) \rightarrow 0$ as $N \rightarrow \infty$ (established in Proposition 2 in Appendix F this residual energy will eventually be collected 

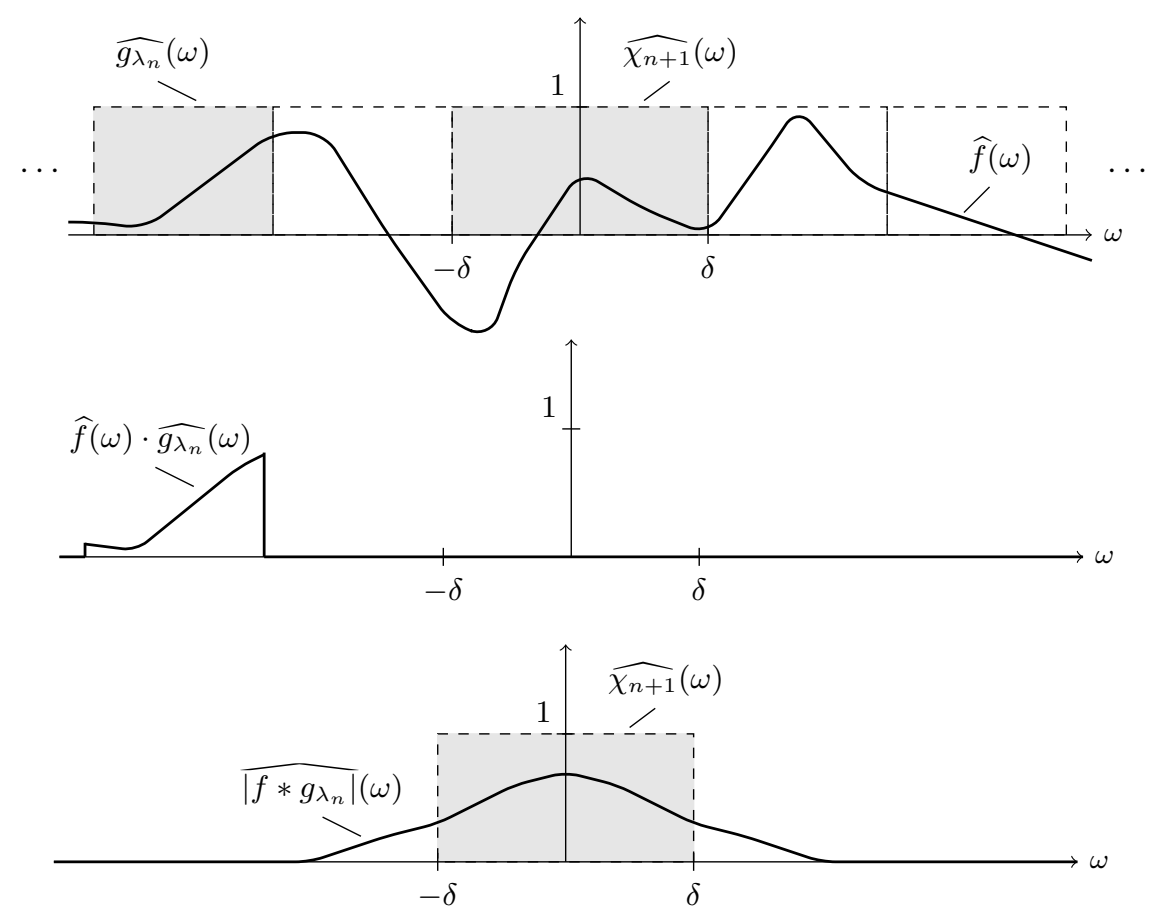

Fig. 5: Illustration of the demodulation effect of the modulus non-linearity. The $\left\{g_{\lambda_{n}}\right\}_{\lambda_{n} \in \Lambda_{n}}$ are taken as perfect band-pass filters (e.g., band-limited analytic Weyl-Heisenberg filters) and hence trivially satisfy the conditions in Assumption 1 The modulus operation in combination with the analyticity and the highpass nature of the filters $\left\{g_{\lambda_{n}}\right\}_{\lambda_{n} \in \Lambda_{n}}$ ensures that-in every network layer-the spectral content of each individual feature map is moved to base-band (i.e., to low frequencies), where it is extracted by the (low-pass) output-generating filter $\chi_{n+1}$.

in the infinite-depth feature vector $\Phi_{\Omega}(f)$ so that no input signal energy is "lost" in the network. In Section V, we shall answer the question of how many layers are needed to absorb $((1-\varepsilon) \cdot 100) \%$ of the input signal energy.

The next result shows that, under additional structural assumptions on the filters $\left\{g_{\lambda_{n}}\right\}_{\lambda_{n} \in \Lambda}$, the guaranteed energy decay rate can be improved from polynomial to exponential. Specifically, we can get exponential energy decay for broad families of wavelets and Weyl-Heisenberg filters. For conceptual reasons, we consider the 1-D case and, for simplicity of exposition, we employ filters that constitute Parseval frames and are identical across network layers.

Theorem 2. Let $\widehat{r}_{l}: \mathbb{R} \rightarrow \mathbb{R}, \widehat{r}_{l}(\omega):=(1-|\omega|)_{+}^{l}$, with $l>1$.

i) Wavelets: Let the mother and father wavelets $\psi, \phi \in$ $L^{1}(\mathbb{R}) \cap L^{2}(\mathbb{R})$ satisfy $\operatorname{supp}(\widehat{\psi}) \subseteq[1 / 2,2]$ and

$$
|\widehat{\phi}(\omega)|^{2}+\sum_{j=1}^{\infty}\left|\widehat{\psi}\left(2^{-j} \omega\right)\right|^{2}=1, \quad \text { a.e. } \omega \geq 0 .
$$

Moreover, let $g_{j}(x):=2^{j} \psi\left(2^{j} x\right)$, for $x \in \mathbb{R}, j \geq 1$, and $g_{j}(x):=2^{|j|} \psi\left(-2^{|j|} x\right)$, for $x \in \mathbb{R}, j \leq-1$, and set $\chi(x):=\phi(x)$, for $x \in \mathbb{R}$. Let $\Omega$ be the module-sequence (11) with filters $\Psi=\{\chi\} \cup\left\{g_{j}\right\}_{j \in \mathbb{Z} \backslash\{0\}}$ in every network layer. Then,

$$
W_{N}(f) \leq \int_{\mathbb{R}}|\widehat{f}(\omega)|^{2}\left(1-\left|\widehat{r}_{l}\left(\frac{\omega}{(5 / 3)^{N-1}}\right)\right|^{2}\right) \mathrm{d} \omega,
$$

for $f \in L^{2}(\mathbb{R})$ and $N \geq 1$. Moreover, for every Sobolev function $f \in H^{s}(\mathbb{R}), s>0$, we have

$$
W_{N}(f)=\mathcal{O}\left((5 / 3)^{-\gamma N}\right),
$$

where $\gamma:=\min \{1,2 s\}$.

ii) Weyl-Heisenberg filters: For $R \in \mathbb{R}$, let the functions $g, \phi \in L^{1}(\mathbb{R}) \cap L^{2}(\mathbb{R})$ satisfy $\operatorname{supp}(\widehat{g}) \subseteq[-R, R]$, $\widehat{g}(-\omega)=\widehat{g}(\omega)$, for $\omega \in \mathbb{R}$, and

$$
|\widehat{\phi}(\omega)|^{2}+\sum_{k=1}^{\infty}|\widehat{g}(\omega-R(k+1))|^{2}=1,
$$

a.e. $\omega \geq 0$. Moreover, let $g_{k}(x):=e^{2 \pi i(k+1) R x} g(x)$, for $x \in \mathbb{R}, k \geq 1$, and $g_{k}(x):=e^{-2 \pi i(|k|+1) R x} g(x)$, for $x \in$ $\mathbb{R}, k \leq-1$, and set $\chi(x):=\phi(x)$, for $x \in \mathbb{R}$. Let $\Omega$ be the module-sequence (1) with filters $\Psi=\{\chi\} \cup\left\{g_{k}\right\}_{k \in \mathbb{Z} \backslash\{0\}}$ in every network layer. Then,

$$
W_{N}(f) \leq \int_{\mathbb{R}}|\widehat{f}(\omega)|^{2}\left(1-\left|\widehat{r}_{l}\left(\frac{\omega}{(3 / 2)^{N-1} R}\right)\right|^{2}\right) \mathrm{d} \omega,
$$

for $f \in L^{2}(\mathbb{R})$ and $N \geq 1$. Moreover, for every Sobolev function $f \in H^{s}(\mathbb{R}), s>0$, we have

$$
W_{N}(f)=\mathcal{O}\left((3 / 2)^{-\gamma N}\right),
$$

where $\gamma:=\min \{1,2 s\}$.

Proof. See Appendix G.

The conditions we impose on the mother and father wavelet $\psi, \phi$ in i) are satisfied, e.g., by analytic Meyer wavelets [22. Section 3.3.5], and those on the prototype function $g$ and low-pass filter $\phi$ in ii) by B-splines [45, Section 1]. Moreover, as shown in [44. Theorem 3.1], the exponential energy decay results in (28) and (31) can be generalized to $\mathcal{O}\left(a^{-N}\right)$ with arbitrary decay factor $a>1$ realized through 
suitable choice of the mother wavelet or the Weyl-Heisenberg prototype function.

We note that in the presence of pooling by sub-sampling (as defined in [9. Eq. 9]), say with pooling factors $S_{n}:=$ $S \in\left[1, a\right.$ ), for all $n \in \mathbb{N}$, (where $a=\frac{5}{3}$ in the wavelet case and $a=\frac{3}{2}$ in the Weyl-Heisenberg case) the effective decay factor in 28 and 31 becomes $\frac{5}{3 S}$ and $\frac{3}{2 S}$, respectively. Exponential energy decay is hence compatible with vertical translation invariance according to [9. Theorem 1], albeit at the cost of a slower (exponential) decay rate. The proof of this statement is structurally very similar to that of Theorem 2 and will therefore not be given here. Finally, we note that the energy decay and conservation results in Theorems 1 and 2 are compatible with the feature extractor $\Phi_{\Omega}$ being deformationinsensitive according to [9, Theorem 2], simply by noting that [9. Theorem 2] applies to general semi-discrete frames and general Lipschitz-continuous non-linearities.

We next put the results in Theorems 1 and 2 into perspective with respect to the literature.

Relation to [10, Section 5]: The basic philosophy of our proof technique for (20), 23, 27), and (30) is inspired by the proof in [10, Section 5], which establishes (9) and (11) for scattering networks based on certain wavelet filters and with $1-\mathrm{D}$ real-valued input signals $f \in L^{2}(\mathbb{R})$. Specifically, in [10, Section 5], in every network layer, the filters $\Psi_{W}=\{\chi\} \cup\left\{g_{j}\right\}_{j \in \mathbb{Z}}$ (where $g_{j}(\omega):=2^{j} \psi\left(2^{j} \omega\right), j \in \mathbb{Z}$, for some mother wavelet $\psi \in L^{1}(\mathbb{R}) \cap L^{2}(\mathbb{R})$ ) are 1-D functions satisfying the frame property (2) with $A_{n}=B_{n}=1, n \in \mathbb{N}$, a mild analyticity condition ${ }^{3}[10$. Eq. 5.5] in the sense of $\left|\widehat{g_{j}}(\omega)\right|, j \in \mathbb{Z}$, being larger for positive frequencies $\omega$ than for the corresponding negative ones, and a vanishing moments condition [10, Eq. 5.6] which controls the behavior of $\widehat{\psi}(\omega)$ around the origin according to $|\widehat{\psi}(\omega)| \leq C|\omega|^{1+\varepsilon}$, for $\omega \in \mathbb{R}$, for some $C, \varepsilon>0$. Similarly to the proof of (11) as given in [10, Section 5], we base our proof of 23 on the energy decomposition identity (24) and on an upper bound on $W_{N}(f)$ (see 9) for the corresponding upper bound established in [10. Section 5]) shown to go to zero as $N \rightarrow \infty$. The exponential energy decay results (21), (28), and (31) for Sobolev functions $f \in H^{s}\left(\mathbb{R}^{d}\right)$ are entirely new. The major differences between [10, Section 5] and our results are (i) that (9) (reported in [10. Section 5]) depends on an unspecified $a>1$, whereas our results in (20), (21), 27), 28), (30), and (31) make the decay factor $a$ and the decay exponent $\alpha$ explicit, (ii) the technical elements employed to arrive at the upper bounds on $W_{N}(f)$; specifically, while the proof in [10, Section 5] makes explicit use of the algebraic structure of the filters, namely, the multiscale structure of wavelets, our proof of (20) is oblivious to the algebraic structure of the filters, which is why it applies to general (possibly unstructured) filters that, in addition, can be different in different network layers, (iii) the assumptions imposed on the filters, namely the analyticity and vanishing moments conditions in [10, Eq. 5.5-5.6], in contrast to our Assumption 1, and (iv) the class of input signals $f$ the results

\footnotetext{
${ }^{3}$ At the time of completion of the present paper, I. Waldspurger kindly sent us a preprint [47] which shows that the analyticity condition [10. Eq. 5.5] on the mother wavelet is not needed for 97 to hold.
}

apply to, namely 1-D real-valued signals in [10, Section 5], and $d$-dimensional complex-valued signals in our Theorem 1

Relation to [11]: For scattering networks that are based on so-called uniform covering filters [11], (10) and (11) are established in [11] for $d$-dimensional complex-valued signals $f \in L^{2}\left(\mathbb{R}^{d}\right)$. Specifically, in [11], in every network layer, the $d$-dimensional filters $\{\chi\} \cup\left\{g_{\lambda}\right\}_{\lambda \in \Lambda}$ are taken to satisfy i) the frame property (2) with $A=B=1$ and hence $A_{n}=B_{n}=1$, $n \in \mathbb{N}$, see [11, Definition 2.1 (c)], ii) a vanishing moments condition [11, Definition 2.1 (a)] according to $\widehat{g_{\lambda}}(0)=0$, for $\lambda \in \Lambda$, and iii) a uniform covering condition [11] Definition 2.1 (b)] which says that the filters' Fourier transform support sets can be covered by a union of finitely many balls. The major differences between [11] and our results are as follows: (i) the results in [11] apply exclusively to filters satisfying the uniform covering condition such as, e.g., Weyl-Heisenberg filters with a band-limited prototype function [11, Proposition 2.3], but do not apply to multi-scale filters such as wavelets, $(\alpha)$-curvelets, shearlets, and ridgelets (see [11, Remark 2.2 (b)]), (ii) [10 as established in [11] leaves the decay factor $a>1$ unspecified, whereas our results in (28) and (31) make the decay factor $a$ explicit (namely, $a=5 / 3$ in the wavelet case and $a=3 / 2$ in the Weyl-Heisenberg case), (iii) the exponential energy decay result in $(10)$ as established in [11] applies to all $f \in L^{2}\left(\mathbb{R}^{d}\right)$ and thus, in particular, to Sobolev input signals (owing to $H^{s}\left(\mathbb{R}^{d}\right) \subseteq L^{2}\left(\mathbb{R}^{d}\right)$, for all $s>0$ ), whereas our decay results in (21), 28), and (31) pertain to Sobolev input signals $f \in H^{s}\left(\mathbb{R}^{d}\right), s>0$, only, (iv) the technical elements employed to arrive at the upper bounds on $W_{N}(f)$, specifically, while the proof in [11] makes explicit use of the uniform covering property of the filters, our proof of 20 is completely oblivious to the (algebraic) structure of the filters, (v) the assumptions imposed on the filters, i.e., the vanishing moments and uniform covering condition in [11. Definition 2.1 (a)-(b)], in contrast to our Assumption 1, which is less restrictive, and thereby makes our results in Theorem 1 apply to general (possibly unstructured) filters that, in addition, can be different in different network layers.

\section{NUMBER OF LAYERS NEEDED}

DCNNs used in practice employ potentially hundreds of layers [7]. Such network depths entail formidable computational challenges both in training and in operating the network. It is therefore important to understand how many layers are needed to have most of the input signal energy be contained in the feature vector. This will be done by considering Parseval frames in all layers, i.e., frames with frame bounds $A_{n}=B_{n}=1$, $n \in \mathbb{N}$. The energy conservation result 23) then implies that the infinite-depth feature vector

$$
\Phi_{\Omega}(f)=\bigcup_{n=0}^{\infty} \Phi_{\Omega}^{n}(f)
$$

contains the entire input signal energy according to $\left\|\mid \Phi_{\Omega}(f)\right\|^{2}=\sum_{n=0}^{\infty}\|\| \Phi_{\Omega}^{n}(f)\left\|^{2}=\right\| f \|_{2}^{2}$. Now, the decomposition 25 reveals that thanks to $\lim _{N \rightarrow \infty} W_{N}(f) \rightarrow 0$, increasing the network depth $N$ implies that the feature vector $\bigcup_{n=0}^{N} \Phi_{\Omega}^{n}(f)$ progressively contains a larger fraction of the 


\begin{tabular}{l|cccccc|} 
& \multicolumn{7}{|c}{$(1-\varepsilon)$} \\
& 0.25 & 0.5 & 0.75 & 0.9 & 0.95 & 0.99 \\
\hline wavelets & 2 & 3 & 4 & 6 & 8 & 11 \\
Weyl-Heisenberg filters & 2 & 4 & 5 & 8 & 10 & 14 \\
general filters & 2 & 3 & 7 & 19 & 39 & 199 \\
\hline
\end{tabular}

Table I: Number $N$ of layers needed to ensure that $((1-\varepsilon) \cdot 100) \%$ of the input signal energy are contained in the features generated in the first $N$ network layers.

input signal energy. We formalize the question on the number of layers needed by asking for bounds of the form

$$
(1-\varepsilon) \leq \frac{\sum_{n=0}^{N}\|\| \Phi_{\Omega}^{n}(f) \|^{2}}{\|f\|_{2}^{2}} \leq 1,
$$

i.e., by determining the network depth $N$ guaranteeing that at least $((1-\varepsilon) \cdot 100) \%$ of the input signal energy are captured by the corresponding depth- $N$ feature vector $\bigcup_{n=0}^{N} \Phi_{\Omega}^{n}(f)$. Moreover, (32) ensures that the depth- $N$ feature extractor $\bigcup_{n=0}^{N} \Phi_{\Omega}^{n}$ exhibits a trivial null-set.

The following results establish handy estimates of the number $N$ of layers needed to guarantee 32. For pedagogical reasons, we start with the case of band-limited input signals and then proceed to a more general statement pertaining to Sobolev functions.

\section{Corollary 1.}

i) Let $\Omega$ be the module-sequence (1) with filters $\left\{g_{\lambda_{n}}\right\}_{\lambda_{n} \in \Lambda_{n}}$ satisfying the conditions in Assumption 1 , and let the corresponding frame bounds be $A_{n}=B_{n}=1, n \in \mathbb{N}$. Let $\delta>0$ be the radius of the spectral gap $B_{\delta}(0)$ left by the filters $\left\{g_{\lambda_{n}}\right\}_{\lambda_{n} \in \Lambda_{n}}$ according to (17). Furthermore, let $l>\lfloor d / 2\rfloor+1, \varepsilon \in(0,1)$, $\alpha$ as defined in (19), and $f \in L^{2}\left(\mathbb{R}^{d}\right)$ L-band-limited. If

$$
N \geq\left\lceil\left(\frac{L}{\left(1-(1-\varepsilon)^{\frac{1}{2 l}}\right) \delta}\right)^{1 / \alpha}-1\right\rceil,
$$

then (32) holds.

ii) Assume that the conditions in Theorem 2 i) and ii) hold. For the wavelet case, let $a=\frac{5}{3}$ and $\delta=1$ (where $\delta$ corresponds to the radius of the spectral gap left by the wavelets $\left.\left\{g_{j}\right\}_{j \in \mathbb{Z} \backslash\{0\}}\right)$. For the Weyl-Heisenberg case, let $a=\frac{3}{2}$ and $\delta=R$ (here, $\delta$ corresponds to the radius of the spectral gap left by the Weyl-Heisenberg filters $\left.\left\{g_{k}\right\}_{k \in \mathbb{Z} \backslash\{0\}}\right)$. Moreover, let $l>1, \varepsilon \in(0,1)$, and $f \in$ $L^{2}(\mathbb{R})$ L-band-limited. If

$$
N \geq\left\lceil\log _{a}\left(\frac{L}{\left(1-(1-\varepsilon)^{\frac{1}{2 l}}\right) \delta}\right)\right\rceil,
$$

then (32) holds in both cases.

Proof. See Appendix $\mathrm{H}$.

Corollary 1 nicely shows how the description complexity of the signal class under consideration, namely the bandwidth $L$ and the dimension $d$ through the decay exponent $\alpha$ defined in (19) determine the number $N$ of layers needed. Specifically, (33) and (34) show that larger bandwidths $L$ and larger dimension $d$ render the input signal $f$ more "complex", which requires deeper networks to capture most of the energy of $f$. The dependence of the lower bounds in 33 and (34) on the network properties, through the module-sequence $\Omega$, is through the decay factor $a>1$ and the radius $\delta$ of the spectral gap left by the filters $\left\{g_{\lambda_{n}}\right\}_{\lambda_{n} \in \Lambda_{n}}$.

The following numerical example provides quantitative insights on the influence of the parameter $\varepsilon$ on (33) and (34). Specifically, we set $L=1, \delta=1, d=1$ (which implies $\alpha=1$, see (19)), $l=1.0001$, and show in Table I the number $N$ of layers needed according to (33) and (34) for different values of $\varepsilon$. The results show that $95 \%$ of the input signal energy are contained in the first 8 layers in the wavelet case and in the first 10 layers in the Weyl-Heisenberg case. We can therefore conclude that in practice a relatively small number of layers is needed to have most of the input signal energy be contained in the feature vector. In contrast, for general filters, where we can guarantee polynomial energy decay only, $N=39$ layers are needed to absorb $95 \%$ of the input signal energy. We hasten to add, however, that 20 simply guarantees polynomial energy decay and does not preclude the energy from decaying faster in practice.

We proceed with the estimates for Sobolev-class input signals.

\section{Corollary 2.}

i) Let $\Omega$ be the module-sequence (1) with filters $\left\{g_{\lambda_{n}}\right\}_{\lambda_{n} \in \Lambda_{n}}$ satisfying the conditions in Assumption 1, and let the corresponding frame bounds be $A_{n}=B_{n}=1, n \in \mathbb{N}$. Let $\delta>0$ be the radius of the spectral gap $B_{\delta}(0)$ left by the filters $\left\{g_{\lambda_{n}}\right\}_{\lambda_{n} \in \Lambda_{n}}$ according to (17). Furthermore, let $l>\lfloor d / 2\rfloor+1, \varepsilon \in(0,1), \alpha$ as defined in (19), and $f \in H^{s}\left(\mathbb{R}^{d}\right) \backslash\{0\}$, for $s>0$. If

$$
N \geq\left\lceil\left(\frac{2 l\|f\|_{H^{s}}^{2 / \gamma}}{\varepsilon^{1 / \gamma} \delta\|f\|_{2}^{2 / \gamma}}\right)^{1 / \alpha}-1\right\rceil,
$$

where $\gamma:=\min \{1,2 s\}$, then (32) holds.

ii) Assume that the conditions in Theorem 2 i) and ii) hold. For the wavelet case, let $a=\frac{5}{3}$ and $\delta=1$ (where $\delta$ corresponds to the radius of the spectral gap left by the wavelets $\left.\left\{g_{j}\right\}_{j \in \mathbb{Z} \backslash\{0\}}\right)$. For the Weyl-Heisenberg case, let $a=\frac{3}{2}$ and $\delta=R$ (here, $\delta$ corresponds to the radius of the spectral gap left by the Weyl-Heisenberg filters $\left.\left\{g_{k}\right\}_{k \in \mathbb{Z} \backslash\{0\}}\right)$. Furthermore, let $l>1, \varepsilon \in(0,1)$, and $f \in H^{s}(\mathbb{R}) \backslash\{0\}$, for $s>0$. If

$$
N \geq\left\lceil\log _{a}\left(\frac{2 l\|f\|_{H^{s}}^{2 / \gamma}}{\varepsilon^{1 / \gamma} \delta\|f\|_{2}^{2 / \gamma}}\right)\right\rceil,
$$

where $\gamma:=\min \{1,2 s\}$, then 32 holds.

Proof. See Appendix I

As already mentioned in Section III Sobolev spaces $H^{s}\left(\mathbb{R}^{d}\right)$ contain a wide range of practically relevant signal classes. The results in Corollary 2 therefore provide-for a wide variety of input signals - a picture of how many layers are needed to have most of the input signal energy be contained in the feature vector. 
The width of the networks considered throughout the paper is, in principle, infinite as the sets $\Lambda_{n}$ need to be countably infinite in order to guarantee that the frame property (2) is satisfied. For input signals that exhibit mild spectral decay, the number of "operationally significant nodes" will, however, be finite in practice. For a treatment of this aspect as well as results on depth-width tradeoffs, the interested reader is referred to $[44]$.

\section{APPENDIX A}

A FEATURE EXTRACTOR WITH A NON-TRIVIAL KERNEL

We show, by way of example, that employing filters $\Psi_{n}$ which satisfy the frame property (2) alone does not guarantee a trivial null-set for the feature extractor $\Phi_{\Omega}$. Specifically, we construct a feature extractor $\Phi_{\Omega}$ based on filters satisfying (2) and a corresponding function $f \neq 0$ with $f \in \mathcal{N}\left(\Phi_{\Omega}\right)$.

Our example employs, in every network layer, filters $\Psi=$ $\{\chi\} \cup\left\{g_{k}\right\}_{k \in \mathbb{Z}}$ that satisfy the Littlewood-Paley condition (18) with $A=B=1$, and where $g_{0}$ is such that $\widehat{g_{0}}(\omega)=1$, for $\omega \in \overline{B_{1}(0)}$, and arbitrary else (of course, as long as the Littlewood-Paley condition (18) with $A=B=1$ is satisfied). We emphasize that no further restrictions are imposed on the filters $\{\chi\} \cup\left\{g_{k}\right\}_{k \in \mathbb{Z}}$, specifically $\chi$ need not be of low-pass nature and the filters $\left\{g_{k}\right\}_{k \in \mathbb{Z}}$ may be structured (such as wavelets [9. Appendix B]) or unstructured (such as random filters [48], [49]), as long as they satisfy the Littlewood-Paley condition (18) with $A=B=1$. Now, consider the input signal $f \in L^{2}\left(\mathbb{R}^{d}\right)$ according to

$$
\widehat{f}(\omega):=(1-|\omega|)_{+}^{l}, \quad \omega \in \mathbb{R}^{d},
$$

with $l>\lfloor d / 2\rfloor+1$. Then $f * g_{0}=f$, owing to $\operatorname{supp}(\widehat{f})=$ $\overline{B_{1}(0)}$ and $\widehat{g_{0}}(\omega)=1$, for $\omega \in \overline{B_{1}(0)}$. Moreover, $\widehat{f}$ is a positive definite radial basis function [50, Theorem 6.20] and hence by [50. Theorem 6.18] $f(x) \geq 0, x \in \mathbb{R}^{d}$, which, in turn, implies $|f|=f$. This yields

$$
U\left[q_{0}^{N}\right] f=|\cdots|\left|f * g_{0}\right| * g_{0}\left|\cdots * g_{0}\right|=f,
$$

for $q_{0}^{N}:=(0,0, \ldots, 0) \in \mathbb{Z}^{N}$ and $N \in \mathbb{N}$. Owing to the energy decomposition identity 24, together with $A_{\Omega}^{N}=B_{\Omega}^{N}=1$, $N \in \mathbb{N}$, which, in turn, is by $A_{n}=B_{n}=1, n \in \mathbb{N}$, we have

$$
\begin{aligned}
& \|f\|_{2}^{2}=\sum_{n=0}^{N-1}\left\|\left|\Phi_{\Omega}^{n}(f) \|\right|^{2}+W_{N}(f)\right. \\
= & \sum_{n=0}^{N-1}\|\| \Phi_{\Omega}^{n}(f)\|\|^{2}+\underbrace{\left\|U\left[q_{0}^{N}\right] f\right\|_{2}^{2}}_{=\|f\|_{2}^{2}}+\sum_{q \in \mathbb{Z}^{N} \backslash\left\{q_{0}^{N}\right\}}\|U[q] f\|_{2}^{2},
\end{aligned}
$$

for $N \in \mathbb{N}$. This implies

$$
\sum_{n=0}^{N-1}\|\| \Phi_{\Omega}^{n}(f)\left\|^{2}+\sum_{q \in \mathbb{Z}^{N} \backslash\left\{q_{0}^{N}\right\}}\right\| U[q] f \|_{2}^{2}=0 .
$$

As both terms in (37) are positive, we can conclude that $\sum_{n=0}^{N-1}\|\| \Phi_{\Omega}^{n}(f) \mid \|^{2}=0, N \in \mathbb{N}$, and thus

$$
\left\|\left|\Phi_{\Omega}(f)\left\|\left.\right|^{2}=\sum_{n=0}^{\infty}\right\|\left\|\Phi_{\Omega}^{n}(f)\right\| \|^{2}=0 .\right.\right.
$$

Since $\left\|\left|\Phi_{\Omega}(f) \|\right|^{2}=0\right.$ implies $\Phi_{\Omega}(f)=0$, we have constructed a non-zero $f$, namely

$$
f(x)=\int_{\mathbb{R}^{d}}(1-|\omega|)_{+}^{l} e^{2 \pi i\langle x, \omega\rangle} \mathrm{d} \omega,
$$

that maps to the all-zeros feature vector, i.e., $f \in \mathcal{N}\left(\Phi_{\Omega}\right)$.

The point of this example is the following. Owing to the nature of $\widehat{g_{0}}(\omega)$ (namely, $\widehat{g_{0}}(\omega)=1$, for $\omega \in \overline{B_{1}(0)}$ ) and the Littlewood-Paley condition

$$
|\widehat{\chi}(\omega)|^{2}+\sum_{k \in \mathbb{Z}}\left|\widehat{g_{k}}(\omega)\right|^{2}=1, \text { a.e. } \omega \in \mathbb{R}^{d},
$$

it follows that neither the output-generating filter $\chi$ nor any of the other filters $g_{k}, k \in \mathbb{Z} \backslash\{0\}$, can have spectral support in $\overline{B_{1}(0)}$. Consequently, the only non-zero contribution to the feature vector can come from

$$
U\left[q_{0}^{N}\right] f=f,
$$

which, however, thanks to $\operatorname{supp}(\widehat{f})=\overline{B_{1}(0)}$, is spectrally disjoint from the output-generating filter $\chi$. Therefore, $\Phi_{\Omega}(f)$ will be identically equal to 0 . Assumption 1 disallows this situation as it forces the filters $g_{k}, k \in \mathbb{Z}$, to be of highpass nature which, in turn, implies that $\chi$ must have lowpass characteristics. The punch-line of our general results on energy conservation, be it for finite $N$ or for $N \rightarrow \infty$, is that Assumption 1 in combination with the frame property and the modulus non-linearity prohibit a non-trivial null-set in general.

\section{APPENDIX B}

\section{SOBOLEV SMOOTHNESS OF CARTOON FUNCTIONS}

Cartoon functions, introduced in [35], satisfy mild decay properties and are piecewise continuously differentiable apart from curved discontinuities along smooth hypersurfaces. This function class has been widely adopted in the mathematical signal processing literature [15], [19], [26], [36], [37] as a standard model for natural images such as, e.g., images of handwritten digits [38] (see Figure 4). We will work with the following — relative to the definition in [35]—-slightly modified version of cartoon functions.

Definition 1. The function $f: \mathbb{R}^{d} \rightarrow \mathbb{C}$ is referred to as a cartoon function if it can be written as $f=f_{1}+\mathbb{1}_{D} f_{2}$, where $D \subseteq \mathbb{R}^{d}$ is a compact domain whose boundary $\partial D$ is a compact topologically embedded $C^{2}$-hypersurface of $\mathbb{R}^{d}$ without boundary $]^{4}$ and $f_{i} \in H^{1 / 2}\left(\mathbb{R}^{d}\right) \cap C^{1}\left(\mathbb{R}^{d}, \mathbb{C}\right), i=1,2$, satisfy the decay condition

$$
\left|\nabla f_{i}(x)\right| \leq C\langle x\rangle^{-d}, \quad i=1,2,
$$

for some $C>0$ (not depending on $f_{1}, f_{2}$ ). Furthermore, we denote by

$$
\begin{aligned}
& \mathcal{C}_{\mathrm{CART}}^{K}:=\left\{f_{1}+\mathbb{1}_{D} f_{2} \mid f_{i} \in H^{1 / 2}\left(\mathbb{R}^{d}\right) \cap C^{1}\left(\mathbb{R}^{d}, \mathbb{C}\right),\right. \\
& \left.\left|\nabla f_{i}(x)\right| \leq K\langle x\rangle^{-d}, \operatorname{vol}^{d-1}(\partial D) \leq K,\left\|f_{2}\right\|_{\infty} \leq K\right\}
\end{aligned}
$$

the class of cartoon functions of "size" $K>0$.

\footnotetext{
${ }^{4}$ We refer the reader to [51 Chapter 0] for a review on differentiable manifolds.
} 
Even though cartoon functions are in general discontinuous, they still admit Sobolev smoothness. The following result formalizes this statement.

Lemma 1. Let $K>0$. Then, $\mathcal{C}_{\mathrm{CART}}^{K} \subseteq H^{s}\left(\mathbb{R}^{d}\right)$, for all $s \in(0,1 / 2)$.

Proof. Let $\left(f_{1}+\mathbb{1}_{D} f_{2}\right) \in \mathcal{C}_{\mathrm{CART}}^{K}$. We first establish $\mathbb{1}_{D} \in$ $H^{s}\left(\mathbb{R}^{d}\right)$, for all $s \in(0,1 / 2)$. To this end, we define the Sobolev-Slobodeckij semi-norm [52, Section 2.1.2]

$$
|f|_{H^{s}}:=\left(\int_{\mathbb{R}^{d}} \int_{\mathbb{R}^{d}} \frac{|f(x)-f(y)|^{2}}{|x-y|^{2 s+d}} \mathrm{~d} x \mathrm{~d} y\right)^{1 / s},
$$

and note that, thanks to [52, Section 2.1.2], $\mathbb{1}_{D} \in H^{s}\left(\mathbb{R}^{d}\right)$ if $\left|\mathbb{1}_{D}\right|_{H^{s}}<\infty$. We have

$$
\begin{aligned}
\left|\mathbb{1}_{D}\right|_{H^{s}}^{s} & =\int_{\mathbb{R}^{d}} \int_{\mathbb{R}^{d}} \frac{\left|\mathbb{1}_{D}(x)-\mathbb{1}_{D}(y)\right|^{2}}{|x-y|^{2 s+d}} \mathrm{~d} x \mathrm{~d} y \\
& =\int_{\mathbb{R}^{d}} \frac{1}{|t|^{2 s+d}} \int_{\mathbb{R}^{d}}\left|\mathbb{1}_{D}(x)-\mathbb{1}_{D}(x-t)\right|^{2} \mathrm{~d} x \mathrm{~d} t,
\end{aligned}
$$

where we employed the change of variables $t=x-y$. Next, we note that, for fixed $t \in \mathbb{R}^{d}$, the function

$$
h_{t}(x):=\left|\mathbb{1}_{D}(x)-\mathbb{1}_{D}(x-t)\right|^{2}
$$

satisfies $h_{t}(x)=1$, for $x \in S_{t}$, where

$$
\begin{aligned}
S_{t}: & =\left\{x \in \mathbb{R}^{d} \mid x \in D \text { and } x-t \notin D\right\} \\
& \cup\left\{x \in \mathbb{R}^{d} \mid x \notin D \text { and } x-t \in D\right\} \\
& =D \Delta(D+t),
\end{aligned}
$$

and $h_{t}(x)=0$, for $x \in \mathbb{R}^{d} \backslash S_{t}$. It follows from 38 that

$$
\operatorname{vol}^{d}\left(S_{t}\right) \leq 2 \operatorname{vol}^{d}(D), \quad \forall t \in \mathbb{R}^{d} .
$$

Moreover, owing to $S_{t} \subseteq\left(\partial D+B_{|t|}(0)\right)$, where $(\partial D+$ $\left.B_{|t|}(0)\right)$ is a tube of radius $|t|$ around the boundary $\partial D$ of $D$ (see Figure 6), and Lemma 2, stated below, there exists a constant $C_{\partial D}>0$ such that

$$
\operatorname{vol}^{d}\left(S_{t}\right) \leq \operatorname{vol}^{d}\left(\partial D+B_{|t|}(0)\right) \leq C_{\partial D}|t|,
$$

for all $t \in \mathbb{R}^{d}$ with $|t| \leq 1$. Next, fix $R$ such that $0<R<1$. Then,

$$
\begin{aligned}
& \left|\mathbb{1}_{D}\right|_{H^{s}}^{s}=\int_{\mathbb{R}^{d}} \frac{1}{|t|^{2 s+d}} \int_{\mathbb{R}^{d}}\left|\mathbb{1}_{D}(x)-\mathbb{1}_{D}(x-t)\right|^{2} \mathrm{~d} x \mathrm{~d} t \\
& =\int_{\mathbb{R}^{d}} \frac{1}{|t|^{2 s+d}} \int_{\mathbb{R}^{d}} h_{t}(x) \mathrm{d} x \mathrm{~d} t \\
& =\int_{\mathbb{R}^{d}} \frac{1}{|t|^{2 s+d}} \int_{S_{t}} 1 \mathrm{~d} x \mathrm{~d} t=\int_{\mathbb{R}^{d}} \frac{\operatorname{vol}^{d}\left(S_{t}\right)}{|t|^{2 s+d}} \mathrm{~d} t \\
& \leq \int_{\mathbb{R}^{d} \backslash B_{R}(0)} \frac{2 \operatorname{vol}^{d}(D)}{|t|^{2 s+d}} \mathrm{~d} t+\int_{B_{R}(0)} \frac{C_{\partial D}}{|t|^{2 s+d-1}} \mathrm{~d} t \\
& =2 \operatorname{vol}^{d}(D) \operatorname{vol}^{d-1}\left(\partial B_{1}(0)\right) \underbrace{\int_{R}^{\infty} r^{-(2 s+1)} \mathrm{d} r}_{=: I_{1}} \\
& +C_{\partial D} \operatorname{vol}^{d-1}\left(\partial B_{1}(0)\right) \underbrace{\int_{0}^{R} r^{-2 s} \mathrm{~d} r}_{=: I_{2}},
\end{aligned}
$$

where in (41) we employed (39) and 40), and in the last

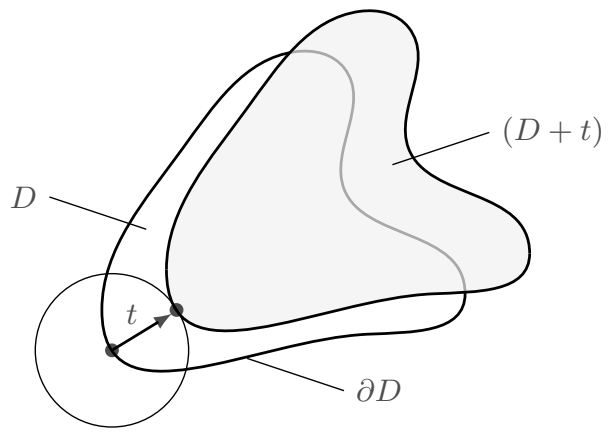

Fig. 6: Illustration in dimension $d=2$. The set $(D+t)$ (grey) is obtained by translating the set $D$ (white) by $t \in \mathbb{R}^{2}$. The symmetric difference $D \Delta(D+t)$ is contained in $\left(\partial D+B_{|t|}(0)\right)$, the tube of radius $|t|$ around the boundary $\partial D$ of $D$.

step we introduced polar coordinates. The integral $I_{1}$ is finite for all $s>0$, while $I_{2}$ is finite for all $s<1 / 2$. Moreover, $\operatorname{vol}^{d}(D)=\int_{D} 1 \mathrm{~d} x$ is finite owing to $D$ being compact (and thus bounded). We can therefore conclude that (42) is finite for $s \in(0,1 / 2)$, and hence $\mathbb{1}_{D} \in H^{s}\left(\mathbb{R}^{d}\right)$, for $s \in(0,1 / 2)$. To see that $\left(f_{1}+\mathbb{1}_{D} f_{2}\right) \in H^{s}\left(\mathbb{R}^{d}\right)$, for $s \in(0,1 / 2)$, we first note that

$$
\left|f_{1}+\mathbb{1}_{D} f_{2}\right|_{H^{s}} \leq\left|f_{1}\right|_{H^{s}}+\left|\mathbb{1}_{D} f_{2}\right|_{H^{s}},
$$

which is thanks to the sub-additivity of the semi-norm $|\cdot|_{H^{s}}$. Now, the first term on the RHS of 43 is finite owing to $f_{1} \in H^{1 / 2}\left(\mathbb{R}^{d}\right) \subseteq H^{s}\left(\mathbb{R}^{d}\right)$, for all $s \in(0,1 / 2)$. For the second term on the RHS, we start by noting that

$$
\begin{aligned}
& \left|\mathbb{1}_{D} f_{2}\right|_{H^{s}}^{s}= \\
& \left(\int_{\mathbb{R}^{d}} \int_{\mathbb{R}^{d}} \frac{\left|\left(\mathbb{1}_{D} f_{2}\right)(x)-\left(\mathbb{1}_{D} f_{2}\right)(y)\right|^{2}}{|x-y|^{2 s+d}} \mathrm{~d} x \mathrm{~d} y\right)
\end{aligned}
$$

and

$$
\begin{aligned}
& \left|\left(\mathbb{1}_{D} f_{2}\right)(x)-\left(\mathbb{1}_{D} f_{2}\right)(y)\right|^{2} \\
& =\left|\left(\mathbb{1}_{D}(x)-\mathbb{1}_{D}(y)\right) f_{2}(x)+\left(f_{2}(x)-f_{2}(y)\right) \mathbb{1}_{D}(y)\right|^{2} \\
& \leq 2\left|\left(\mathbb{1}_{D}(x)-\mathbb{1}_{D}(y)\right)\right|^{2}\left|f_{2}(x)\right|^{2} \\
& +2\left|\left(f_{2}(x)-f_{2}(y)\right)\right|^{2}\left|\mathbb{1}_{D}(y)\right|^{2}
\end{aligned}
$$

where (45) and (46) are thanks to $|a+b|^{2} \leq 2|a|^{2}+2|b|^{2}$, for $a, b \in \mathbb{C}$. Substituting (45) and (46) into (44) and noting that $\left|f_{2}(x)\right|^{2} \leq\left\|f_{2}\right\|_{\infty}^{2} \leq K^{2}, x \in \mathbb{R}^{d}$, which is by assumption, and $\mathbb{1}_{D}(y) \leq 1, y \in \mathbb{R}^{d}$, implies

$$
\left|\mathbb{1}_{D} f_{2}\right|_{H^{s}}^{s} \leq 2 K^{2}\left|\mathbb{1}_{D}\right|_{H^{s}}^{s}+2\left|f_{2}\right|_{H^{s}}^{s}<\infty,
$$

where in the last step we used $\mathbb{1}_{D} \in H^{s}\left(\mathbb{R}^{d}\right)$, established above, and $f_{2} \in H^{1 / 2}\left(\mathbb{R}^{d}\right) \subseteq H^{s}\left(\mathbb{R}^{d}\right)$, both for all $s \in$ $(0,1 / 2)$. This completes the proof.

It remains to establish the second inequality in 40 .

Lemma 2. Let $M$ be a compact topologically embedded $C^{2}$ hypersurface of $\mathbb{R}^{d}$ without boundary and let

$$
T(M, r):=\left\{x \in \mathbb{R}^{d}\left|\inf _{y \in M}\right| x-y \mid \leq r\right\}, \quad r>0,
$$

be the tube of radius $r$ around $M$. Then, there exists a constant 
$C_{M}>0$ (that does not depend on $r$ ) such that for all $r \leq 1$ it holds that

$$
\operatorname{vol}^{d}(T(M, r)) \leq C_{M} r .
$$

Proof. The proof is based on Weyl's tube formula [54]. Let

$$
\kappa:=\max _{i \in\{1, \ldots, d-1\}} \kappa_{i},
$$

where $\kappa_{i}$ is the $i$-th principal curvature of the hypersurface $M$ (see [53. Section 3.1] for a formal definition). It follows from [53, Theorem 8.4 (i)] that

$$
\operatorname{vol}^{d}(T(M, r))=\sum_{i=0}^{\left\lfloor\frac{d-1}{2}\right\rfloor} \frac{2 r^{2 i+1} k_{2 i}(M)}{\prod_{j=0}^{i}(1+2 j)},
$$

for all $r \leq \kappa^{-1}$, where $k_{2 i}(M)=\int_{M} H_{2 i}(x) \mathrm{d} x, i \in$ $\left\{0, \ldots,\left\lfloor\frac{d-1}{2}\right\rfloor\right\}$, with $H_{2 i}$ denoting the so-called $(2 i)$-th curvature of $M$, see [53, Section 4.1] for a formal definition. Now, thanks to $M$ being a $C^{2}$-hypersurface, we have that $H_{2 i}, i \in\left\{0, \ldots,\left\lfloor\frac{d-1}{2}\right\rfloor\right\}$, is bounded (see [53. Section 4.1]), which together with $M$ compact (and thus bounded) implies $\left|k_{2 i}(M)\right|<\infty$, for all $i \in\left\{0, \ldots,\left\lfloor\frac{d-1}{2}\right\rfloor\right\}$. Moreover, by definition, $k_{2 i}(M), i \in\left\{0, \ldots,\left\lfloor\frac{d-1}{2}\right\rfloor\right\}$, is independent of the tube radius $r$. Therefore, setting

$$
C_{M}:=\left(\left\lfloor\frac{d-1}{2}\right\rfloor+1\right) \cdot \max _{i} \frac{2\left|k_{2 i}(M)\right|}{\prod_{j=0}^{i}(1+2 j)}
$$

establishes (48) for $0<r \leq \min \left\{1, \kappa^{-1}\right\}$. It remains to prove (48) for $\min \left\{1, \kappa^{-1}\right\}<r \leq 1$. Let

$$
R^{*}:=\inf \left\{R>0: M \subseteq B_{R}(0)\right\}
$$

and $D_{R^{*}}:=\operatorname{vol}^{d}\left(B_{R^{*}+1}(0)\right)$. Since

$$
\operatorname{vol}^{d}(T(M, r)) \leq D_{R^{*}}, \quad \forall 0<r \leq 1,
$$

it follows that

$$
\operatorname{vol}^{d}(T(M, r))<D_{R^{*}} \cdot \max \{1, \kappa\} \cdot r
$$

for all $\min \left\{1, \kappa^{-1}\right\}<r \leq 1$, which establishes (48) for $\min \left\{1, \kappa^{-1}\right\}<r \leq 1$ and thereby concludes the proof.

\section{APPENDIX C}

PROOF OF STATEMENT I) IN THEOREM 1

We start by establishing 20 with $\alpha=$ $\log _{2}(\sqrt{d /(d-1 / 2)})$, for $d \geq 1$. Then, we sharpen our result in the 1-D case by proving that holds for $d=1$ with $\alpha=1$. This leads to a significant improvement, in the 1-D case, of the decay exponent from $\log _{2}(\sqrt{d /(d-1 / 2)})=\frac{1}{2}$ to 1. The idea for the proof of 20, for $\alpha=\log _{2}(\sqrt{d /(d-1 / 2)})$, $d \geq 1$, is to establish that 5

$$
\begin{gathered}
\sum_{q \in \Lambda_{n} \times \Lambda_{n+1} \times \cdots \times \Lambda_{n+N-1}}\|U[q] f\|_{2}^{2} \\
\leq C_{n}^{n+N-1} \int_{\mathbb{R}^{d}}|\widehat{f}(\omega)|^{2}\left(1-\left|\widehat{r_{l}}\left(\frac{\omega}{N^{\alpha} \delta}\right)\right|^{2}\right) \mathrm{d} \omega,
\end{gathered}
$$

\footnotetext{
${ }^{5}$ We prove the more general result 49 for technical reasons, concretely in order to be able to argue by induction over path lengths with flexible starting index $n$.
}

for $N \in \mathbb{N}$, where

$$
C_{n}^{n+N-1}:=\prod_{k=n}^{n+N-1} \max \left\{1, B_{k}\right\} .
$$

Setting $n=1$ in (49) and noting that $C_{1}^{N}=B_{\Omega}^{N}$ yields the desired result 20. We proceed by induction over the path length $\ell(q):=N$, for $q=\left(\lambda_{n}, \lambda_{n+1}, \ldots, \lambda_{n+N-1}\right) \in \Lambda_{n} \times$ $\Lambda_{n+1} \times \cdots \times \Lambda_{n+N-1}$. Starting with the base case $N=1$, we have

$$
\begin{aligned}
& \sum_{q \in \Lambda_{n}}\|U[q] f\|_{2}^{2}=\sum_{\lambda_{n} \in \Lambda_{n}}\left\|f * g_{\lambda_{n}}\right\|_{2}^{2} \\
& =\int_{\mathbb{R}^{d}} \sum_{\lambda_{n} \in \Lambda_{n}}\left|\widehat{g_{\lambda_{n}}}(\omega)\right|^{2}|\widehat{f}(\omega)|^{2} \mathrm{~d} \omega \\
& \leq B_{n} \int_{\mathbb{R}^{d} \backslash B_{\delta}(0)}|\widehat{f}(\omega)|^{2} \mathrm{~d} \omega \\
& \leq \underbrace{\max \left\{1, B_{n}\right\}}_{=C_{n}^{n}} \int_{\mathbb{R}^{d}}|\widehat{f}(\omega)|^{2}\left(1-\left|\widehat{r_{l}}\left(\frac{\omega}{\delta}\right)\right|^{2}\right) \mathrm{d} \omega,
\end{aligned}
$$

for $N \in \mathbb{N}$, where (50) is by Parseval's formula, (51) is thanks to (17) and (18), and (52) is due to $\operatorname{supp}\left(\widehat{r}_{l}\right) \subseteq B_{1}(0)$ and $0 \leq \widehat{r}_{l}(\omega) \leq 1$, for $\omega \in \mathbb{R}^{d}$. The inductive step is established as follows. Let $N>1$ and suppose that (49) holds for all paths $q$ of length $\ell(q)=N-1$, i.e.,

$$
\begin{gathered}
\sum_{q \in \Lambda_{n} \times \Lambda_{n+1} \times \cdots \times \Lambda_{n+N-2}}\|U[q] f\|_{2}^{2} \\
\leq C_{n}^{n+N-2} \int_{\mathbb{R}^{d}}|\widehat{f}(\omega)|^{2}\left(1-\left|\widehat{r}_{l}\left(\frac{\omega}{(N-1)^{\alpha} \delta}\right)\right|^{2}\right) \mathrm{d} \omega,
\end{gathered}
$$

for $n \in \mathbb{N}$. We start by noting that every path $\tilde{q} \in \Lambda_{n} \times \Lambda_{n+1} \times$ $\ldots \times \Lambda_{n+N-1}$ of length $\ell(\tilde{q})=N$, with arbitrary starting index $n$, can be decomposed into a path $q \in \Lambda_{n+1} \times \ldots \times \Lambda_{n+N-1}$ of length $\ell(q)=N-1$ and an index $\lambda_{n} \in \Lambda_{n}$ according to $\tilde{q}=\left(\lambda_{n}, q\right)$. Thanks to (4) we have

$$
U[\tilde{q}]=U\left[\left(\lambda_{n}, q\right)\right]=U[q] U_{n}\left[\lambda_{n}\right],
$$

which yields

$$
\begin{gathered}
\sum_{q \in \Lambda_{n} \times \Lambda_{n+1} \times \cdots \times \Lambda_{n+N-1}}\|U[q] f\|_{2}^{2} \\
=\sum_{\lambda_{n} \in \Lambda_{n}} \sum_{q \in \Lambda_{n+1} \times \cdots \times \Lambda_{n+N-1}}\left\|U[q]\left(U_{n}\left[\lambda_{n}\right] f\right)\right\|_{2}^{2},
\end{gathered}
$$

for $n \in \mathbb{N}$. We proceed by examining the inner sum on the RHS of (54). Invoking the induction hypothesis (53) with $n$ replaced by $(n+1)$ and employing Parseval's formula, we get

$$
\begin{aligned}
& \sum_{q \in \Lambda_{n+1} \times \cdots \times \Lambda_{n+N-1}}\left\|U[q]\left(U_{n}\left[\lambda_{n}\right] f\right)\right\|_{2}^{2} \\
\leq & C_{n+1}^{n+N-1} \int_{\mathbb{R}^{d}}\left|\widehat{U_{n}\left[\lambda_{n}\right] f}(\omega)\right|^{2}\left(1-\left|\widehat{r_{l}}\left(\frac{\omega}{(N-1)^{\alpha} \delta}\right)\right|^{2}\right) \mathrm{d} \omega \\
= & C_{n+1}^{n+N-1}\left(\left\|U_{n}\left[\lambda_{n}\right] f\right\|_{2}^{2}-\left\|\left(U_{n}\left[\lambda_{n}\right] f\right) * r_{l, N-1, \alpha, \delta}\right\|_{2}^{2}\right) \\
= & C_{n+1}^{n+N-1}\left(\left\|f * g_{\lambda_{n}}\right\|_{2}^{2}-\left\|f * g_{\lambda_{n}} \mid * r_{l, N-1, \alpha, \delta}\right\|_{2}^{2}\right),
\end{aligned}
$$

for $n \in \mathbb{N}$, where $r_{l, N-1, \alpha, \delta}$ is the inverse Fourier transform of $\widehat{r}_{l}\left(\frac{\omega}{(N-1)^{\alpha} \delta}\right)$. Next, we note that $\widehat{r}_{l}\left(\frac{\omega}{(N-1)^{\alpha} \delta}\right)$ is a positive definite radial basis function [50, Theorem 6.20] and hence 
by [50, Theorem 6.18] $r_{l, N-1, \alpha, \delta}(x) \geq 0$, for $x \in \mathbb{R}^{d}$. Furthermore, it follows from Lemma 3, stated below, that for $\left\{\nu_{\lambda_{n}}\right\}_{\lambda_{n} \in \Lambda_{n}} \subseteq \mathbb{R}^{d}$, we have

$$
\begin{aligned}
& \left\|f * g_{\lambda_{n}} \mid * r_{l, N-1, \alpha, \delta}\right\|_{2}^{2} \\
& \geq\left\|f * g_{\lambda_{n}} *\left(M_{\nu_{\lambda_{n}}} r_{l, N-1, \alpha, \delta}\right)\right\|_{2}^{2} .
\end{aligned}
$$

Here, we note that choosing the modulation factors $\left\{\nu_{\lambda_{n}}\right\}_{\lambda_{n} \in \Lambda_{n}} \subseteq \mathbb{R}^{d}$ appropriately (see 600 below) will be key in establishing the inductive step.

Lemma 3. [8. Lemma 2.7]: Let $f, g \in L^{2}\left(\mathbb{R}^{d}\right)$ with $g(x) \geq 0$, for $x \in \mathbb{R}^{d}$. Then, $\||f| * g\|_{2}^{2} \geq\left\|f *\left(M_{\omega} g\right)\right\|_{2}^{2}$, for $\omega \in \mathbb{R}^{d}$.

Inserting (55) and 56) into the inner sum on the RHS of (54) yields

$$
\begin{aligned}
& \sum_{q \in \Lambda_{n} \times \Lambda_{n+1} \times \cdots \times \Lambda_{n+N-1}}\|U[q] f\|_{2}^{2} \\
\leq & C_{n+1}^{n+N-1} \sum_{\lambda_{n} \in \Lambda_{n}}\left(\left\|f * g_{\lambda_{n}}\right\|_{2}^{2}\right. \\
- & \left.\left\|f * g_{\lambda_{n}} *\left(M_{\nu_{\lambda_{n}}} r_{l, N-1, \alpha, \delta}\right)\right\|_{2}^{2}\right) \\
= & C_{n+1}^{n+N-1} \int_{\mathbb{R}^{d}}|\widehat{f}(\omega)|^{2} h_{n, N, \alpha, \delta}(\omega) \mathrm{d} \omega, \quad \forall N \in \mathbb{N},
\end{aligned}
$$

where we applied Parseval's formula together with $\widehat{M_{\omega} f}=$ $T_{\omega} \widehat{f}$, for $f \in L^{2}\left(\mathbb{R}^{d}\right)$, and $\omega \in \mathbb{R}^{d}$, and set

$$
\begin{aligned}
& h_{n, N, \alpha, \delta}(\omega) \\
& :=\sum_{\lambda_{n} \in \Lambda_{n}}\left|\widehat{g_{\lambda_{n}}}(\omega)\right|^{2}\left(1-\left|\widehat{r}_{l}\left(\frac{\omega-\nu_{\lambda_{n}}}{(N-1)^{\alpha} \delta}\right)\right|^{2}\right) .
\end{aligned}
$$

The key step is now to establish-by judiciously choosing $\left\{\nu_{\lambda_{n}}\right\}_{\lambda_{n} \in \Lambda_{n}} \subseteq \mathbb{R}^{d}$-the upper bound

$$
h_{n, N, \alpha, \delta}(\omega) \leq \max \left\{1, B_{n}\right\}\left(1-\left|\widehat{r}_{l}\left(\frac{\omega}{N^{\alpha} \delta}\right)\right|^{2}\right),
$$

for $\omega \in \mathbb{R}^{d}$, which upon noting that $C_{n}^{n+N-1}=$ $\max \left\{1, B_{n}\right\} C_{n+1}^{n+N-1}$ yields (49) and thereby completes the proof. We start by defining $H_{A_{\lambda_{n}}}$, for $\lambda_{n} \in \Lambda_{n}$, to be the orthant supporting $\widehat{g_{\lambda_{n}}}$, i.e., $\operatorname{supp}\left(\widehat{g_{\lambda_{n}}}\right) \subseteq H_{A_{\lambda_{n}}}$, where $A_{\lambda_{n}} \in O(d)$, for $\lambda_{n} \in \Lambda_{n}$ (see Assumption 1). Furthermore, for $\lambda_{n} \in \Lambda_{n}$, we choose the modulation factors according to

$$
\nu_{\lambda_{n}}:=A_{\lambda_{n}} \nu \in \mathbb{R}^{d},
$$

where the components of $\nu \in \mathbb{R}^{d}$ are given by $\nu_{k}:=(1+$ $\left.2^{-1 / 2}\right) \frac{\delta}{d}$, for $k \in\{1, \ldots, d\}$. Invoking (16) and (17), we get

$$
\begin{aligned}
& h_{n, N, \alpha, \delta}(\omega)=\sum_{\lambda_{n} \in \Lambda_{n}}\left|\widehat{g_{\lambda_{n}}}(\omega)\right|^{2}\left(1-\left|\widehat{r_{l}}\left(\frac{\omega-\nu_{\lambda_{n}}}{(N-1)^{\alpha} \delta}\right)\right|^{2}\right) \\
& =\sum_{\lambda_{n} \in \Lambda_{n}}\left|\widehat{g_{\lambda_{n}}}(\omega)\right|^{2} \mathbb{1}_{S_{\lambda_{n}, \delta}}(\omega)\left(1-\left|\widehat{r_{l}}\left(\frac{\omega-\nu_{\lambda_{n}}}{(N-1)^{\alpha} \delta}\right)\right|^{2}\right),
\end{aligned}
$$

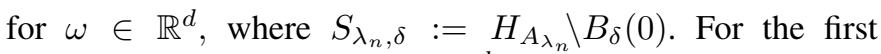
canonical orthant $H=\left\{x \in \mathbb{R}^{d} \mid x_{k} \geq 0, k=1, \ldots, d\right\}$, we show in Lemma 4 below that

$$
\left|\widehat{r}_{l}\left(\frac{\omega-\nu}{(N-1)^{\alpha} \delta}\right)\right| \geq\left|\widehat{r}_{l}\left(\frac{\omega}{N^{\alpha} \delta}\right)\right|,
$$

for $\omega \in H \backslash B_{\delta}(0)$ and $N \geq 2$. This will allow us to deduce

$$
\left|\widehat{r}_{l}\left(\frac{\omega-\nu_{\lambda_{n}}}{(N-1)^{\alpha} \delta}\right)\right| \geq\left|\widehat{r}_{l}\left(\frac{\omega}{N^{\alpha} \delta}\right)\right|,
$$

for $\omega \in S_{\lambda_{n}, \delta}, \lambda_{n} \in \Lambda_{n}$, and $N \geq 2$, where $S_{\lambda_{n}, \delta}=$ $H_{A_{\lambda_{n}}} \backslash B_{\delta}(0)$, simply by noting that

$$
\begin{aligned}
& \left|\widehat{r}_{l}\left(\frac{\omega-\nu_{\lambda_{n}}}{(N-1)^{\alpha} \delta}\right)\right|=\left(1-\left|\frac{A_{\lambda_{n}}\left(\omega^{\prime}-\nu\right)}{(N-1)^{\alpha} \delta}\right|\right)_{+}^{l} \\
& =\left(1-\left|\frac{\omega^{\prime}-\nu}{(N-1)^{\alpha} \delta}\right|\right)_{+}^{l}=\left|\widehat{r}_{l}\left(\frac{\omega^{\prime}-\nu}{(N-1)^{\alpha} \delta}\right)\right| \\
& \geq\left|\widehat{r}_{l}\left(\frac{\omega^{\prime}}{N^{\alpha} \delta}\right)\right|=\left(1-\left|\frac{\omega^{\prime}}{N^{\alpha} \delta}\right|\right)_{+}^{l} \\
& =\left(1-\left|\frac{A_{\lambda_{n}} \omega^{\prime}}{N^{\alpha} \delta}\right|\right)_{+}^{l}=\left|\widehat{r}_{l}\left(\frac{\omega}{N^{\alpha} \delta}\right)\right|,
\end{aligned}
$$

for $\omega=A_{\lambda_{n}} \omega^{\prime} \in H_{A_{\lambda_{n}}} \backslash B_{\delta}(0)$, where $\omega^{\prime} \in H \backslash B_{\delta}(0)$. Here, (64) and (66) are thanks to $|\omega|=\left|A_{\lambda_{n}} \omega\right|$, which is by $A_{\lambda_{n}} \in$ $O(d)$, and the inequality in (65) is due to (62). Insertion of (63) into (61) then yields

$$
\begin{aligned}
h_{n, N, \alpha, \delta}(\omega) & \leq \sum_{\lambda_{n} \in \Lambda_{n}}\left|\widehat{g_{\lambda_{n}}}(\omega)\right|^{2} \mathbb{1}_{S_{\lambda_{n}, \delta}}(\omega)\left(1-\left|\widehat{r_{l}}\left(\frac{\omega}{N^{\alpha} \delta}\right)\right|^{2}\right) \\
& =\sum_{\lambda_{n} \in \Lambda_{n}}\left|\widehat{g_{\lambda_{n}}}(\omega)\right|^{2}\left(1-\left|\widehat{r_{l}}\left(\frac{\omega}{N^{\alpha} \delta}\right)\right|^{2}\right) \\
& \leq \max \left\{1, B_{n}\right\}\left(1-\left|\widehat{r}_{l}\left(\frac{\omega}{N^{\alpha} \delta}\right)\right|^{2}\right)
\end{aligned}
$$

for $\omega \in \mathbb{R}^{d}$, where in (67) we employed Assumption 1, and (68) is thanks to (18). This establishes (59) and completes the proof of (20) for $\alpha=\log _{2}(\sqrt{d /(d-1 / 2)}), d \geq 1$.

It remains to show (62), which is accomplished through the following lemma.

Lemma 4. Let $\alpha:=\log _{2}(\sqrt{d /(d-1 / 2)}), \widehat{r}_{l}: \mathbb{R}^{d} \rightarrow \mathbb{R}$, $\widehat{r}_{l}(\omega):=(1-|\omega|)_{+}^{l}$, with $l>\lfloor d / 2\rfloor+1$, and define $\nu \in \mathbb{R}^{d}$ to have components $\nu_{k}=\left(1+2^{-1 / 2}\right) \frac{\delta}{d}$, for $k \in\{1, \ldots, d\}$. Then,

$$
\left|\widehat{r}_{l}\left(\frac{\omega-\nu}{(N-1)^{\alpha} \delta}\right)\right| \geq\left|\widehat{r}_{l}\left(\frac{\omega}{N^{\alpha} \delta}\right)\right|,
$$

for $\omega \in H \backslash B_{\delta}(0)$ and $N \geq 2$.

Proof. The key idea of the proof is to employ a monotonicity argument. Specifically, thanks to $\widehat{r}_{l}$ monotonically decreasing in $|\omega|$, i.e., $\widehat{r}_{l}\left(\omega_{1}\right) \geq \widehat{r}_{l}\left(\omega_{2}\right)$, for $\omega_{1}, \omega_{2} \in \mathbb{R}^{d}$ with $\left|\omega_{2}\right| \geq\left|\omega_{1}\right|$, (69) can be established simply by showing that

$$
\kappa_{N}(\omega):=|\omega|^{2}\left|\frac{N-1}{N}\right|^{2 \alpha}-|\omega-\nu|^{2} \geq 0,
$$

for $\omega \in H \backslash B_{\delta}(0)$ and $N \geq 2$. We first note that for $\omega \in$ $H \backslash B_{\delta}(0)$ with $|\omega|>N^{\alpha} \delta$, (69) is trivially satisfied as the RHS of 699) equals zero (owing to $\left|\frac{\omega}{N^{\alpha} \delta}\right|>1$ together with $\left.\operatorname{supp}\left(\widehat{r}_{l}\right) \subseteq B_{1}(0)\right)$. It hence suffices to prove (70) for $\omega \in H$ with $\delta \leq|\omega| \leq N^{\alpha} \delta$. To this end, fix $\tau \in\left[\delta, N^{\alpha} \delta\right]$, and define the spherical segment

$$
\Xi_{\tau}:=\{\omega \in H|| \omega \mid=\tau\} .
$$




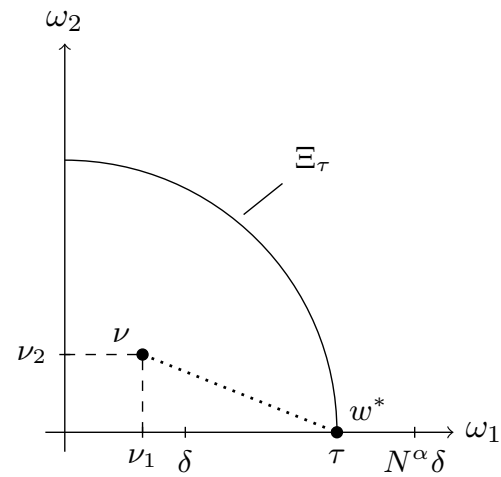

Fig. 7: Illustration of (71) in dimension $d=2$. The mapping $\omega \mapsto|\omega-\nu|^{2}$, $\omega \in \Xi_{\tau}=\left\{\omega=\left(\omega_{1}, \omega_{2}\right) \in \mathbb{R}^{2}|| \omega \mid=\tau, \omega_{1} \geq 0, \omega_{2} \geq 0\right\}$, computes the squared Euclidean distance between an element $\omega$ of the spherical segment $\Xi_{\tau}$ and the vector $\nu=\left(\nu_{1}, \nu_{2}\right)$ with components $\nu_{k}=\left(1+2^{-1 / 2}\right) \frac{\delta}{2}$, $k \in\{1,2\}$. The mapping attains its maxima along the coordinate axes, e.g., for $\omega^{*}=(\tau, 0) \in \Xi_{\tau}$.

We then have

$$
\begin{aligned}
\kappa_{N}(\omega) & =\tau^{2}\left|\frac{N-1}{N}\right|^{2 \alpha}-|\omega-\nu|^{2} \\
& \geq \tau^{2}\left|\frac{N-1}{N}\right|^{2 \alpha}-\left|\omega^{*}-\nu\right|^{2},
\end{aligned}
$$

for $\omega \in \Xi_{\tau}$ and $N \geq 2$, where

$$
\omega^{*}=(\tau, 0, \ldots, 0) \in \Xi_{\tau} .
$$

The inequality in 71 holds thanks to the mapping

$$
\omega \mapsto|\omega-\nu|^{2}, \quad \omega \in \Xi_{\tau},
$$

attaining its maxima along the coordinate axes (see Figure 7 . Inserting

$$
\begin{aligned}
\left|\omega^{*}-\nu\right|^{2} & =\left(\tau-\frac{\delta\left(1+2^{-1 / 2}\right)}{d}\right)^{2}+\frac{(d-1) \delta^{2}\left(1+2^{-1 / 2}\right)^{2}}{d^{2}} \\
& =\tau^{2}-\frac{\tau \delta\left(2+2^{1 / 2}\right)}{d}+\frac{\delta^{2}\left(1+2^{-1 / 2}\right)^{2}}{d}
\end{aligned}
$$

into 71 and rearranging terms yields

$$
\underbrace{\kappa_{N}(\omega)}_{=: p_{N}(\tau)} \underbrace{\tau^{2}\left(\left|\frac{N-1}{N}\right|^{2 \alpha}-1\right)+\frac{\tau \delta\left(2+2^{1 / 2}\right)}{d}-\frac{\delta^{2}\left(1+2^{-1 / 2}\right)^{2}}{d}},
$$

for $\omega \in \Xi_{\tau}$ and $N \geq 2$. This inequality shows that $\kappa_{N}(\omega)$ is lower-bounded-for $\omega \in \Xi_{\tau}$-by the 1-D function $p_{N}(\tau)$. Now, $p_{N}(\tau)$ is quadratic in $\tau$, with the highest-degree coefficient

$$
\left(\left|\frac{N-1}{N}\right|^{2 \alpha}-1\right)
$$

negative owing to

$$
\alpha=\log _{2}(\sqrt{d /(d-1 / 2)})>0, \quad \forall d \geq 1 .
$$

Therefore, thanks to $p_{N}, N \geq 2$, being concave, establishing $p_{N}(\delta) \geq 0$ and $p_{N}\left(N^{\alpha} \delta\right) \geq 0$, for $N \geq 2$, implies $p_{N}(\tau) \geq 0$, for $\tau \in\left[\delta, N^{\alpha} \delta\right]$ and $N \geq 2$ (see Figure 8 , and thus [70],

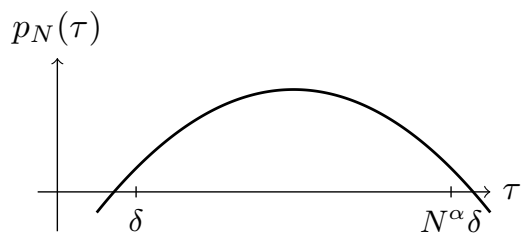

Fig. 8: The function $p_{N}(\tau)$ is quadratic in $\tau$, with the coefficient of the highest-degree term negative. Establishing $p_{N}(\delta) \geq 0$ and $p_{N}\left(N^{\alpha} \delta\right) \geq 0$ therefore implies $p_{N}(\tau) \geq 0, \tau \in\left[\delta, N^{\alpha} \delta\right]$.

which completes the proof. It remains to show that $p_{N}(\delta) \geq 0$ and $p_{N}\left(N^{\alpha} \delta\right) \geq 0$, both for $N \geq 2$. We have

$$
\begin{aligned}
p_{N}(\delta) & =\delta^{2}\left(\left|\frac{N-1}{N}\right|^{2 \alpha}-1+\frac{2+2^{1 / 2}}{d}-\frac{\left(1+2^{-1 / 2}\right)^{2}}{d}\right) \\
& \geq \delta^{2}\left(2^{-2 \alpha}-\frac{d-1 / 2}{d}\right)=0,
\end{aligned}
$$

where the inequality in 72 is by

$$
N \mapsto\left|\frac{N-1}{N}\right|^{2 \alpha}, \quad N \geq 2,
$$

monotonically increasing in $N$, and the equality is thanks to $\alpha=\log _{2}(\sqrt{d /(d-1 / 2)})$. Next, we have

$$
\begin{aligned}
& \frac{p_{N}\left(N^{\alpha} \delta\right)}{\delta^{2}} \\
& =|N-1|^{2 \alpha}-N^{2 \alpha}+\frac{N^{\alpha}\left(2+2^{1 / 2}\right)}{d}-\frac{\left(1+2^{-1 / 2}\right)^{2}}{d} \\
& \geq 1-2^{2 \alpha}+\frac{2^{\alpha}\left(2+2^{1 / 2}\right)}{d}-\frac{\left(1+2^{-1 / 2}\right)^{2}}{d} \\
& =1-\frac{d}{d-1 / 2}+\frac{\sqrt{d}\left(2+2^{1 / 2}\right)}{d \sqrt{d-1 / 2}}-\frac{\left(1+2^{-1 / 2}\right)^{2}}{d} \geq 0,
\end{aligned}
$$

for $d \geq 1$ and $N \geq 2$, where (73) is by $N \mapsto(N-$ $1)^{2 \alpha}-N^{2 \alpha}+d^{-1} N^{\alpha}\left(2+2^{1 / 2}\right)$, for $N \geq 2$, monotonically increasing in $N$ (owing to $\alpha=\log _{2}(\sqrt{d /(d-1 / 2)})>0$, for $d \geq 1$ ), and the equality in (74) is thanks to $\alpha=$ $\log _{2}(\sqrt{d /(d-1 / 2)})$. The inequality in (74) is established in Lemma 5 below. This completes the proof.

Lemma 5. For every $d \geq 1$ it holds that

$$
1-\frac{d}{d-1 / 2}+\frac{\sqrt{d}\left(2+2^{1 / 2}\right)}{d \sqrt{d-1 / 2}}-\frac{\left(1+2^{-1 / 2}\right)^{2}}{d} \geq 0 .
$$

Proof. We start by multiplying the inequality by $d(d-1 / 2)$, which (after rearranging terms) yields

$$
\sqrt{d(d-1 / 2)} \alpha \geq(d-1 / 2) \beta+d / 2, \quad d \geq 1,
$$

where

$$
\alpha:=\left(2+2^{1 / 2}\right), \quad \beta:=\left(1+2^{-1 / 2}\right)^{2} .
$$

Squaring (75) yields (again, after rearranging terms)

$$
d^{2} \underbrace{\left(\alpha^{2}-\beta^{2}-\beta-\frac{1}{4}\right)}_{=0}+d \underbrace{\left(-\frac{\alpha^{2}}{2}+\beta^{2}+\frac{\beta}{2}\right)}_{\geq 4}-\underbrace{\frac{\beta^{2}}{4}}_{\geq 3} \geq 0
$$


for $d \geq 1$, which completes the proof.

We proceed to sharpen, for $d=1$, the exponent $\alpha=$ $\log _{2}(\sqrt{d /(d-1 / 2)})=1 / 2$ to $\alpha=1$. The structure of the corresponding proof is similar to that of the proof of the general result $\alpha=\log _{2}(\sqrt{d /(d-1 / 2)})$, for $d \geq 1$. Specifically, we start by employing the arguments leading to 57) with $N^{\alpha}$ replaced by $N$. With this replacement $h_{n, N, \alpha, \delta}$ in (58) becomes

$$
h_{n, N, \alpha, \delta}(\omega):=\sum_{\lambda_{n} \in \Lambda_{n}}\left|\widehat{g_{\lambda_{n}}}(\omega)\right|^{2}\left(1-\left|\widehat{r_{l}}\left(\frac{\omega-\nu_{\lambda_{n}}}{(N-1) \delta}\right)\right|^{2}\right),
$$

where, again, appropriate choice of the modulation factors $\left\{\nu_{\lambda_{n}}\right\}_{\lambda_{n} \in \Lambda_{n}} \subseteq \mathbb{R}^{d}$ will be key in establishing the inductive step. We start by defining $\Lambda_{n}^{+}$to be the set of indices $\lambda_{n} \in \Lambda_{n}$ such that $\operatorname{supp}\left(\widehat{g_{\lambda_{n}}}\right) \subseteq[\delta, \infty)$, and take $\Lambda_{n}^{-}$to be the set of indices $\lambda_{n} \in \Lambda_{n}$ so that $\operatorname{supp}\left(\widehat{g_{\lambda_{n}}}\right) \subseteq(-\infty,-\delta]$ (see Assumption 11. Clearly, $\Lambda_{n}=\Lambda_{n}^{+} \cup \Lambda_{n}^{-}$. Moreover, we define the modulation factors according to $\nu_{\lambda_{n}}:=\delta$, for $\lambda_{n} \in \Lambda_{n}^{+}$, and $\nu_{\lambda_{n}}:=-\delta$, for $\lambda_{n} \in \Lambda_{n}^{-}$. We then get

$$
\begin{aligned}
& h_{n, N, \alpha, \delta}(\omega)=\sum_{\lambda_{n} \in \Lambda_{n}}\left|\widehat{g_{\lambda_{n}}}(\omega)\right|^{2}\left(1-\left|\widehat{r_{l}}\left(\frac{\omega-\nu_{\lambda_{n}}}{(N-1) \delta}\right)\right|^{2}\right) \\
& =\sum_{\lambda_{n} \in \Lambda_{n}^{+}}\left|\widehat{g_{\lambda_{n}}}(\omega)\right|^{2} \mathbb{1}_{[\delta, \infty)}(\omega)\left(1-\left|\widehat{r_{l}}\left(\frac{\omega-\delta}{(N-1) \delta}\right)\right|^{2}\right) \\
& +\sum_{\lambda_{n} \in \Lambda_{n}^{-}}\left|\widehat{g_{\lambda_{n}}}(\omega)\right|^{2} \mathbb{1}_{(-\infty,-\delta]}(\omega)\left(1-\left|\widehat{r_{l}}\left(\frac{\omega+\delta}{(N-1) \delta}\right)\right|^{2}\right) \\
& \leq \max _{\{}\left\{1, B_{n}\right\} \mathbb{1}_{[\delta, \infty)}(\omega)\left(1-\left|\widehat{r_{l}}\left(\frac{\omega-\delta}{(N-1) \delta}\right)\right|^{2}\right) \\
& +\max \left\{1, B_{n}\right\} \mathbb{1}_{(-\infty,-\delta]}(\omega)\left(1-\left|\widehat{r}_{l}\left(\frac{\omega+\delta}{(N-1) \delta}\right)\right|^{2}\right),
\end{aligned}
$$

where (76) is thanks to Assumption 1, and for the last step we employed (18). We show in Lemma 6 below that

$$
\left|\widehat{r}_{l}\left(\frac{\omega-\delta}{(N-1) \delta}\right)\right| \geq\left|\widehat{r}_{l}\left(\frac{\omega}{N \delta}\right)\right|,
$$

for $\omega \in[\delta, \infty)$ and $N \geq 2$. This will allow us to deduce

$$
\left|\widehat{r}_{l}\left(\frac{\omega+\delta}{(N-1) \delta}\right)\right| \geq\left|\widehat{r}_{l}\left(\frac{\omega}{N \delta}\right)\right|
$$

for $\omega \in(-\infty,-\delta]$ and $N \geq 2$, simply by noting that

$$
\begin{aligned}
& \left|\widehat{r}_{l}\left(\frac{\omega+\delta}{(N-1) \delta}\right)\right|=\left(1-\left|\frac{\omega+\delta}{(N-1) \delta}\right|\right)_{+}^{l} \\
& =\left(1-\left|\frac{-(-\omega-\delta)}{(N-1) \delta}\right|\right)_{+}^{l}=\left|\widehat{r}_{l}\left(\frac{-\omega-\delta}{(N-1) \delta}\right)\right| \\
& \geq\left|\widehat{r}_{l}\left(\frac{-\omega}{N \delta}\right)\right|=\left(1-\left|\frac{-\omega}{N \delta}\right|\right)_{+}^{l}=\left|\widehat{r}_{l}\left(\frac{\omega}{N \delta}\right)\right|,
\end{aligned}
$$

for $\omega \in(-\infty,-\delta]$. Here, the inequality in 81 is due to 79 ). Insertion of (79) into (77) and of (80) into (78) then yields

$$
\begin{aligned}
& h_{n, N, \alpha, \delta}(\omega) \\
& \leq \max \left\{1, B_{n}\right\} \mathbb{1}_{(-\infty, \delta] \cup[\delta, \infty)}(\omega)\left(1-\left|\widehat{r}_{l}\left(\frac{\omega}{N \delta}\right)\right|^{2}\right) \\
& \leq \max \left\{1, B_{n}\right\}\left(1-\left|\widehat{r}_{l}\left(\frac{\omega}{N \delta}\right)\right|^{2}\right),
\end{aligned}
$$

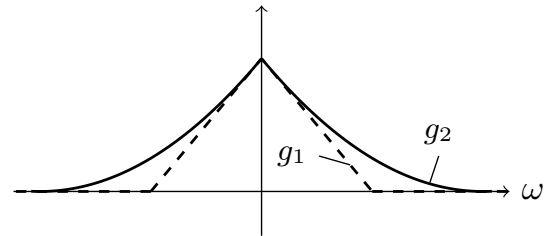

Fig. 9: Illustration of 86 in dimension $d=1$. The functions $g_{1}(\omega):=$ $\max \{0,1-2 l|\omega|\}$ (dashed line) and $g_{2}(\omega):=(1-|\omega|)_{+}^{2 l}$ (solid line) satisfy $g_{1}(\omega) \leq g_{2}(\omega)$, for $\omega \in \mathbb{R}$. Note that $l>\lfloor d / 2\rfloor+1$.

for $\omega \in \mathbb{R}$, where the last inequality is thanks to $0 \leq \widehat{r}_{l}(\omega) \leq$ 1 , for $\omega \in \mathbb{R}$. This establishes 20 -in the 1 -D case-for $\alpha=1$ and completes the proof of statement i) in Theorem 1

It remains to prove (79), which is done through the following lemma.

Lemma 6. Let $\widehat{r_{l}}: \mathbb{R} \rightarrow \mathbb{R}, \widehat{r}_{l}(\omega):=(1-|\omega|)_{+}^{l}$, with $l>1$. Then,

$$
\left|\widehat{r}_{l}\left(\frac{\omega-\delta}{(N-1) \delta}\right)\right| \geq\left|\widehat{r}_{l}\left(\frac{\omega}{N \delta}\right)\right|
$$

for $\omega \in[\delta, \infty)$ and $N \geq 2$.

Proof. We first note that for $\omega>N \delta$, (82) is trivially satisfied as the RHS of (82) equals zero (owing to $\left|\frac{\omega}{N \delta}\right|>1$ together with $\left.\operatorname{supp}\left(\widehat{r}_{l}\right) \subseteq B_{1}(0)\right)$. It hence suffices to prove (82) for $\delta \leq \omega \leq N \delta$. The key idea of the proof is to employ a monotonicity argument. Specifically, thanks to $\widehat{r}_{l}$ monotonically decreasing in $|\omega|$, i.e., $\widehat{r}_{l}\left(\omega_{1}\right) \geq \widehat{r}_{l}\left(\omega_{2}\right)$, for $\omega_{1}, \omega_{2} \in \mathbb{R}$ with $\left|\omega_{2}\right| \geq\left|\omega_{1}\right|$, [82 can be established simply by showing that

$$
\left|\frac{\omega-\delta}{(N-1) \delta}\right| \leq\left|\frac{\omega}{N \delta}\right|, \quad \forall \omega \in[\delta, N \delta], \forall N \geq 2,
$$

which, by $\omega \in[\delta, N \delta]$, is equivalent to

$$
\frac{\omega-\delta}{(N-1) \delta} \leq \frac{\omega}{N \delta}, \quad \forall \omega \in[\delta, N \delta], \forall N \geq 2
$$

Rearranging terms in 83 , we get $\omega \leq N \delta$, for $\omega \in[\delta, N \delta]$ and $N \geq 2$, which completes the proof.

Remark 1. What makes the improved exponent $\alpha$ possible in the 1-D case is the absence of rotated orthants. Specifically, for $d=1$, the filters $\left\{g_{\lambda_{n}}\right\}_{\lambda_{n} \in \Lambda_{n}}$ satisfy either $\operatorname{supp}\left(\widehat{g_{\lambda_{n}}}\right) \subseteq$ $(-\infty,-\delta]$ or $\operatorname{supp}\left(\widehat{g_{\lambda_{n}}}\right) \subseteq[\delta, \infty)$, i.e., the support sets $\operatorname{supp}\left(\widehat{g_{\lambda_{n}}}\right)$ are located in one of the two half-spaces.

\section{APPENDIX D \\ PRoOF OF STATEMENT II) IN THEOREM 1}

We need to show that there exist constants $C_{1, s}, C_{2, s}>0$ (that are independent of $N$ ) such that

$$
W_{N}(f) \leq C_{1, s} B_{\Omega}^{N} N^{-2 s \alpha}, \quad \forall s \in(0,1 / 2), \forall N \geq 1,
$$

and

$$
W_{N}(f) \leq C_{2, s} B_{\Omega}^{N} N^{-\alpha}, \quad \forall s \in[1 / 2, \infty), \forall N \geq 1 .
$$

Let us start by noting that

$$
\max \{0,1-2 l|\omega|\} \leq(1-|\omega|)_{+}^{2 l}, \quad \omega \in \mathbb{R}^{d},
$$


where $l>\lfloor d / 2\rfloor+1$, see Figure 9 This implies

$$
\begin{aligned}
1-\left|\widehat{r}_{l}\left(\frac{\omega}{N^{\alpha} \delta}\right)\right|^{2} & =1-\left(1-\left|\frac{\omega}{N^{\alpha} \delta}\right|\right)_{+}^{2 l} \\
& \leq 1-\max \left\{0,1-\frac{2 l|\omega|}{N^{\alpha} \delta}\right\} \\
& =1+\min \left\{0, \frac{2 l|\omega|}{N^{\alpha} \delta}-1\right\} \\
& =\min \left\{1, \frac{2 l|\omega|}{N^{\alpha} \delta}\right\}, \quad \forall \omega \in \mathbb{R}^{d} .
\end{aligned}
$$

The key idea of the proof of (84) is to upper-bound the integral on the RHS of 20 according to

$$
\begin{aligned}
& \int_{\mathbb{R}^{d}}|\widehat{f}(\omega)|^{2}\left(1-\left|\widehat{r_{l}}\left(\frac{\omega}{N^{\alpha} \delta}\right)\right|^{2}\right) \mathrm{d} \omega \\
& \leq \int_{\mathbb{R}^{d}}|\widehat{f}(\omega)|^{2} \min \left\{1, \frac{2 l|\omega|}{N^{\alpha} \delta}\right\} \mathrm{d} \omega \\
& =\int_{B_{\tau}(0)}|\widehat{f}(\omega)|^{2} \frac{2 l|\omega|}{N^{\alpha} \delta} \mathrm{d} \omega+\int_{\mathbb{R}^{d} \backslash B_{\tau}(0)}^{|\widehat{f}(\omega)|^{2} \mathrm{~d} \omega,}
\end{aligned}
$$

where $\tau:=\frac{N^{\alpha} \delta}{2 l}$. Here, the inequality in 88 follows from (87), and 89 is owing to

$$
\min \left\{1, \frac{2 l|\omega|}{N^{\alpha} \delta}\right\}= \begin{cases}\frac{2 l|\omega|}{N^{\alpha} \delta}, & |\omega| \leq \tau \\ 1, & |\omega|>\tau .\end{cases}
$$

Now, the first integral in 89 satisfies

$$
\begin{aligned}
& \int_{B_{\tau}(0)}|\widehat{f}(\omega)|^{2} \frac{2 l|\omega|}{N^{\alpha} \delta} \mathrm{d} \omega=\frac{2 l}{N^{\alpha} \delta} \int_{B_{\tau}(0)}|\widehat{f}(\omega)|^{2}|\omega|^{1-2 s}|\omega|^{2 s} \mathrm{~d} \omega \\
& \leq \frac{2 l \tau^{1-2 s}}{N^{\alpha} \delta} \int_{B_{\tau}(0)}|\widehat{f}(\omega)|^{2}|\omega|^{2 s} \mathrm{~d} \omega \\
& \leq \frac{2 l \tau^{1-2 s}}{N^{\alpha} \delta} \int_{B_{\tau}(0)}|\widehat{f}(\omega)|^{2}\left(1+|\omega|^{2}\right)^{s} \mathrm{~d} \omega \\
& \leq\left(\frac{2 l}{N^{\alpha} \delta}\right)^{2 s} \int_{B_{\tau}(0)}|\widehat{f}(\omega)|^{2}\left(1+|\omega|^{2}\right)^{s} \mathrm{~d} \omega
\end{aligned}
$$

where 90 is owing to $|\omega| \mapsto(1+|\omega|)^{1-2 s}$ monotonically increasing in $|\omega|$ for $s \in(0,1 / 2)$. For the second integral in (89), we have

$$
\begin{aligned}
& \int_{\mathbb{R}^{d} \backslash B_{\tau}(0)}|\widehat{f}(\omega)|^{2} \mathrm{~d} \omega=\int_{\mathbb{R}^{d} \backslash B_{\tau}(0)}|\widehat{f}(\omega)|^{2}|\omega|^{-2 s}|\omega|^{2 s} \mathrm{~d} \omega \\
& \leq \tau^{-2 s} \int_{\mathbb{R}^{d} \backslash B_{\tau}(0)}|\widehat{f}(\omega)|^{2} \underbrace{|\omega|^{2 s}}_{\leq\left(1+|\omega|^{2}\right)^{s}} \mathrm{~d} \omega \\
& \leq \tau^{-2 s} \int_{\mathbb{R}^{d} \backslash B_{\tau}(0)}|\widehat{f}(\omega)|^{2}\left(1+|\omega|^{2}\right)^{s} \mathrm{~d} \omega \\
& \leq\left(\frac{2 l}{N^{\alpha} \delta}\right)^{2 s} \int_{\mathbb{R}^{d} \backslash B_{\tau}(0)}|\widehat{f}(\omega)|^{2}\left(1+|\omega|^{2}\right)^{s} \mathrm{~d} \omega,
\end{aligned}
$$

where 92 is thanks to

$$
|\omega| \mapsto|\omega|^{-2 s}, \quad \omega \in \mathbb{R}^{d},
$$

monotonically decreasing in $|\omega|$ for $s \in(0,1 / 2)$. Inserting 91) and 93 into (89) establishes 84 with

$$
C_{1, s}:=(2 l)^{2 s} \delta^{-2 s}\|f\|_{H^{s}}^{2} .
$$

Next, we show 85 by noting that

$$
\begin{aligned}
& \int_{\mathbb{R}^{d}}|\widehat{f}(\omega)|^{2}\left(1-\left|\widehat{r_{l}}\left(\frac{\omega}{N^{\alpha} \delta}\right)\right|^{2}\right) \mathrm{d} \omega \\
& \leq \int_{\mathbb{R}^{d}}|\widehat{f}(\omega)|^{2} \min \left\{1, \frac{2 l|\omega|}{N^{\alpha} \delta}\right\} \mathrm{d} \omega \\
& \leq \frac{2 l}{N^{\alpha} \delta} \int_{\mathbb{R}^{d}}|\widehat{f}(\omega)|^{2}|\omega| \mathrm{d} \omega \\
& \leq \frac{2 l}{N^{\alpha} \delta} \int_{\mathbb{R}^{d}}|\widehat{f}(\omega)|^{2}\left(1+|\omega|^{2}\right)^{s} \mathrm{~d} \omega=\frac{2 l}{N^{\alpha} \delta}\|f\|_{H^{s}}^{2},
\end{aligned}
$$

where 94 is by (87), and the last inequality follows from $|\omega| \leq\left(1+|\omega|^{2}\right)^{s}$, for $\omega \in \mathbb{R}^{d}$ and $s \in[1 / 2, \infty)$. This establishes 85 with

$$
C_{2, s}:=(2 l) \delta^{-1}\|f\|_{H^{s}}^{2}
$$

and thereby completes the proof.

\section{APPENDIX E \\ PROPOSITION 1}

Proposition 1. Let $\Omega$ be the module-sequence (1). Then,

$$
A_{\Omega}^{N}\|f\|_{2}^{2} \leq \sum_{n=0}^{N-1}\left\|\Phi_{\Omega}^{n}(f)\right\|\left\|^{2}+W_{N}(f) \leq B_{\Omega}^{N}\right\| f \|_{2}^{2},
$$

for all $f \in L^{2}\left(\mathbb{R}^{d}\right)$ and all $N \geq 1$, where $A_{\Omega}^{N}=$ $\prod_{k=1}^{N} \min \left\{1, A_{k}\right\}$ and $B_{\Omega}^{N}=\prod_{k=1}^{N} \max \left\{1, B_{k}\right\}$.

Proof. We proceed by induction over $N$ and start with the base case $N=1$ which follows directly from the frame property (2) according to

$$
\begin{aligned}
A_{\Omega}^{1}\|f\|_{2}^{2} & =\min \left\{1, A_{1}\right\}\|f\|_{2}^{2} \leq A_{1}\|f\|_{2}^{2} \\
& \leq \underbrace{\|f\|^{2}+W_{1}(f)}_{=\left\|f * \chi_{1}\right\|_{2}^{2}+\sum_{\lambda_{1} \in \Lambda_{1}}\left\|f * \Phi_{\lambda_{1}}\right\|_{2}^{2}} \leq B_{1}\|f\|_{2}^{2} \\
& \leq \max \left\{1, B_{1}\right\}\|f\|_{2}^{2}=B_{\Omega}^{1}\|f\|_{2}^{2}, \quad \forall f \in L^{2}\left(\mathbb{R}^{d}\right) .
\end{aligned}
$$

The inductive step is obtained as follows. Let $N>1$ and suppose that 95 holds for $N-1$, i.e.,

$$
\begin{aligned}
A_{\Omega}^{N-1}\|f\|_{2}^{2} & \leq \sum_{n=0}^{N-2}\left\|\Phi_{\Omega}^{n}(f)\right\|^{2}+W_{N-1}(f) \\
& \leq B_{\Omega}^{N-1}\|f\|_{2}^{2}, \quad \forall f \in L^{2}\left(\mathbb{R}^{d}\right) .
\end{aligned}
$$

We start by noting that

$$
\begin{aligned}
& \sum_{n=0}^{N-1}\left\|\mid \Phi_{\Omega}^{n}(f)\right\|\left\|^{2}+W_{N}(f)=\sum_{n=0}^{N-2}\right\| \Phi_{\Omega}^{n}(f)\|\|^{2} \\
& +\sum_{q \in \Lambda^{N-1}}\left\|(U[q] f) * \chi_{N}\right\|_{2}^{2}+\sum_{q \in \Lambda^{N}}\|U[q] f\|_{2}^{2},
\end{aligned}
$$

and proceed by examining the third term on the RHS of (97). Every path

$$
\tilde{q} \in \Lambda^{N}=\underbrace{\Lambda_{1} \times \ldots \times \Lambda_{N-1}}_{=\Lambda^{N-1}} \times \Lambda_{N}
$$

of length $N$ can be decomposed into a path $q \in \Lambda^{N-1}$ of length $N-1$ and an index $\lambda_{N} \in \Lambda_{N}$ according to $\tilde{q}=\left(q, \lambda_{N}\right)$. 
Thanks to (4) we have $U[\tilde{q}]=U\left[\left(q, \lambda_{N}\right)\right]=U_{N}\left[\lambda_{N}\right] U[q]$, which yields

$$
\sum_{q \in \Lambda^{N}}\|U[q] f\|_{2}^{2}=\sum_{q \in \Lambda^{N-1}} \sum_{\lambda_{N} \in \Lambda_{N}}\left\|(U[q] f) * g_{\lambda_{N}}\right\|_{2}^{2} .
$$

Substituting the third term on the RHS of 977 by (98) and rearranging terms, we obtain

$$
\begin{aligned}
& \sum_{n=0}^{N-1}\left\|\mid \Phi_{\Omega}^{n}(f)\right\|\left\|^{2}+W_{N}(f)=\sum_{n=0}^{N-2}\right\| \Phi_{\Omega}^{n}(f)\|\|^{2} \\
& +\sum_{q \in \Lambda^{N-1}}(\underbrace{\left\|(U[q] f) * \chi_{N}\right\|_{2}^{2}+\sum_{\lambda_{N} \in \Lambda_{N}}\left\|(U[q] f) * g_{\lambda_{N}}\right\|_{2}^{2}}_{=: \rho_{N}(U[q] f)}) .
\end{aligned}
$$

Thanks to the frame property (2) and $U[q] f \in L^{2}\left(\mathbb{R}^{d}\right)$, which is by [9. Eq. 16], we have $A_{N}\|U[q] f\|_{2}^{2} \leq \rho_{N}(U[q] f) \leq$ $B_{N}\|U[q] f\|_{2}^{2}$, and thus

$$
\begin{aligned}
& \min \left\{1, A_{N}\right\}\left(\left.\sum_{n=0}^{N-2}\left\||| \Phi_{\Omega}^{n}(f)\right\|\right|^{2}+W_{N-1}(f)\right) \\
& \leq \sum_{n=0}^{N-1}\left\|\mid \Phi_{\Omega}^{n}(f)\right\|^{2}+W_{N}(f) \\
& \leq \max \left\{1, B_{N}\right\}\left(\sum_{n=0}^{N-2}\left|\left\|\Phi_{\Omega}^{n}(f) \mid\right\|^{2}+W_{N-1}(f)\right),\right.
\end{aligned}
$$

where we employed the identity $\sum_{q \in \Lambda^{N-1}}\|U[q] f\|_{2}^{2}=$ $W_{N-1}(f)$. Invoking the induction hypothesis (96) in 99) and $(100)$ and noting that $A_{\Omega}^{N}=\min \left\{1, A_{N}\right\} A_{\Omega}^{N-1}$ and $B_{\Omega}^{N}=\max \left\{1, B_{N}\right\} B_{\Omega}^{N-1}$ completes the proof.

\section{APPENDIX F}

\section{PROPOSITION 2}

Proposition 2. Let $\widehat{r}_{l}: \mathbb{R}^{d} \rightarrow \mathbb{R}, \widehat{r}_{l}(\omega):=(1-|\omega|)_{+}^{l}$, with $l>\lfloor d / 2\rfloor+1$, and let $\alpha:=\log _{2}(\sqrt{d /(d-1 / 2)})$. Then, we have

$$
\lim _{N \rightarrow \infty} \int_{\mathbb{R}^{d}}|\widehat{f}(\omega)|^{2}\left(1-\left|\widehat{r}_{l}\left(\frac{\omega}{N^{\alpha} \delta}\right)\right|^{2}\right) \mathrm{d} \omega=0,
$$

for all $f \in L^{2}\left(\mathbb{R}^{d}\right)$.

Proof. We start by setting

$$
d_{N, \alpha, \delta}(\omega):=\left(1-\left|\widehat{r}_{l}\left(\frac{\omega}{N^{\alpha} \delta}\right)\right|^{2}\right), \quad \omega \in \mathbb{R}^{d}, N \in \mathbb{N} .
$$

Let $f \in L^{2}\left(\mathbb{R}^{d}\right)$. For every $\varepsilon>0$ there exists $R>0$ such that

$$
\int_{\mathbb{R}^{d} \backslash \overline{B_{R}(0)}}|\widehat{f}(\omega)|^{2} \mathrm{~d} \omega \leq \varepsilon / 2,
$$

where $\overline{B_{R}(0)}$ denotes the closed ball of radius $R$ centered at the origin. Next, we employ Dini's theorem [55, Theorem 7.3] to show that $\left(d_{N, \alpha, \delta}\right)_{N \in \mathbb{N}}$ converges to zero uniformly on $\overline{B_{R}(0)}$. To this end, we note that (i) $d_{N, \alpha, \delta}$ is continuous as a composition of continuous functions, (ii) $z_{0}(\omega)=0$, for $\omega \in \mathbb{R}^{d}$, is clearly continuous, (iii) $d_{N, \alpha, \delta}(\omega) \geq d_{N+1, \alpha, \delta}(\omega)$, for $\omega \in \mathbb{R}^{d}$ and $N \in \mathbb{N}$, and (iv) $d_{N, \alpha, \delta}$ converges to $z_{0}$ pointwise on $\overline{B_{R}(0)}$, i.e.,

$$
\lim _{N \rightarrow \infty} d_{N, \alpha, \delta}(\omega)=z_{0}(\omega)=0, \quad \forall \omega \in \mathbb{R}^{d} .
$$

This allows us to conclude that there exists $N_{0} \in \mathbb{N}$ (that depends on $\varepsilon$ ) such that $d_{N, \alpha, \delta}(\omega) \leq \frac{\varepsilon}{2\|f\|_{2}^{2}}$, for $\omega \in \overline{B_{R}(0)}$ and $N \geq N_{0}$, and we therefore get

$$
\begin{aligned}
\int_{\mathbb{R}^{d}}|\widehat{f}(\omega)|^{2} d_{N, \alpha, \delta}(\omega) \mathrm{d} \omega & =\int_{\mathbb{R}^{d} \backslash \overline{B_{R}(0)}}|\widehat{f}(\omega)|^{2} \underbrace{d_{N, \alpha, \delta}(\omega)}_{\leq 1} \mathrm{~d} \omega \\
& +\int_{\overline{B_{R}(0)}}|\widehat{f}(\omega)|^{2} \underbrace{d_{N, \alpha, \delta}(\omega)}_{\leq \frac{\varepsilon}{2\|f\|_{2}^{2}}} \mathrm{~d} \omega \\
& \leq \frac{\varepsilon}{2}+\frac{\varepsilon}{2\|f\|_{2}^{2}}\|\widehat{f}\|_{2}^{2}=\varepsilon,
\end{aligned}
$$

where in the last step we employed Parseval's formula. Since $\varepsilon>0$ was arbitrary, we have (101), which completes the proof.

\section{APPENDIX G \\ PROOF OF THEOREM 2}

We start by establishing (27) in statement i). The structure of the proof is similar to that of the proof of statement i) in Theorem 1, specifically we perform induction over $N$. Starting with the base case $N=1$, we first note that $\operatorname{supp}(\widehat{\psi}) \subseteq$ $[1 / 2,2], \widehat{g_{j}}(\omega)=\widehat{\psi}\left(2^{-j} \omega\right), j \geq 1$, and $\widehat{g_{j}}(\omega)=\widehat{\psi}\left(-2^{-|j|} \omega\right)$, $j \leq-1$, all by assumption, imply

$$
\operatorname{supp}\left(\widehat{g_{j}}\right)=\operatorname{supp}\left(\widehat{\psi}\left(2^{-j} \cdot\right)\right) \subseteq\left[2^{j-1}, 2^{j+1}\right], \quad j \geq 1,
$$

and

$$
\operatorname{supp}\left(\widehat{g_{j}}\right)=\operatorname{supp}\left(\widehat{\psi}\left(-2^{-|j|} .\right)\right) \subseteq\left[-2^{|j|+1},-2^{|j|-1}\right],
$$

for $j \leq-1$. We then get

$$
\begin{aligned}
W_{1}(f) & =\sum_{j \in \mathbb{Z} \backslash\{0\}}\left\|f * g_{j}\right\|_{2}^{2} \\
& =\int_{\mathbb{R}} \sum_{j \in \mathbb{Z} \backslash\{0\}}\left|\widehat{g_{j}}(\omega)\right|^{2}|\widehat{f}(\omega)|^{2} \mathrm{~d} \omega \\
& =\int_{\mathbb{R}} \sum_{j \geq 1}\left|\widehat{\psi}\left(2^{-j} \omega\right)\right|^{2}|\widehat{f}(\omega)|^{2} \mathrm{~d} \omega \\
& +\int_{\mathbb{R}} \sum_{j \leq-1}\left|\widehat{\psi}\left(-2^{-|j|} \omega\right)\right|^{2}|\widehat{f}(\omega)|^{2} \mathrm{~d} \omega \\
& =\int_{1}^{\infty} \sum_{j \geq 1}\left|\widehat{\psi}\left(2^{-j} \omega\right)\right|^{2}|\widehat{f}(\omega)|^{2} \mathrm{~d} \omega \\
& +\int_{-\infty}^{-1} \sum_{j \leq-1}\left|\widehat{\psi}\left(-2^{-|j|} \omega\right)\right|^{2}|\widehat{f}(\omega)|^{2} \mathrm{~d} \omega \\
& \leq \int_{\mathbb{R} \backslash[-1,1]}|\widehat{f}(\omega)|^{2} \mathrm{~d} \omega \\
& \leq \int_{\mathbb{R}}|\widehat{f}(\omega)|^{2}\left(1-\left|\widehat{r_{l}}(\omega)\right|^{2}\right) \mathrm{d} \omega,
\end{aligned}
$$

where (104) is by Parseval's formula, and (105) is thanks to (102) and (103). The inequality in (106) is owing to 26), and 107) is due to $\operatorname{supp}\left(\widehat{r}_{l}\right) \subseteq[-1,1]$ and $0 \leq \widehat{r}_{l}(\omega) \leq 1$, for 
$\omega \in \mathbb{R}$. The inductive step is obtained as follows. Let $N>1$ and suppose that 27) holds for $N-1$, i.e.,

$$
W_{N-1}(f) \leq \int_{\mathbb{R}}|\widehat{f}(\omega)|^{2}\left(1-\left|\widehat{r}_{l}\left(\frac{\omega}{(5 / 3)^{N-2}}\right)\right|^{2}\right) \mathrm{d} \omega
$$

for $f \in L^{2}(\mathbb{R})$. We start by noting that every path $\tilde{q} \in$ $(\mathbb{Z} \backslash\{0\})^{N}$ of length $N$ can be decomposed into a path $q \in(\mathbb{Z} \backslash\{0\})^{N-1}$ of length $N-1$ and an index $j \in \mathbb{Z} \backslash\{0\}$ according to $\tilde{q}=(j, q)$. Thanks to (4) we have $U[\tilde{q}]=$ $U[(j, q)]=U[q] U_{1}[j]$, which yields

$$
\begin{aligned}
W_{N}(f) & =\sum_{j \in \mathbb{Z} \backslash\{0\}} \sum_{q \in(\mathbb{Z} \backslash\{0\})^{N-1}}\left\|U[q]\left(U_{1}[j] f\right)\right\|_{2}^{2} \\
& =\sum_{j \in \mathbb{Z} \backslash\{0\}} W_{N-1}\left(U_{1}[j] f\right) .
\end{aligned}
$$

We proceed by examining the term $W_{N-1}\left(U_{1}[j] f\right)$ inside the sum on the RHS of (109). Invoking the induction hypothesis (108) and employing Parseval's formula, we get

$$
\begin{aligned}
& W_{N-1}\left(U_{1}[j] f\right) \\
& \leq \int_{\mathbb{R}}\left|\widehat{U_{1}[j] f}(\omega)\right|^{2}\left(1-\left|\widehat{r_{l}}\left(\frac{\omega}{(5 / 3)^{N-2}}\right)\right|^{2}\right) \mathrm{d} \omega \\
& =\left(\left\|U_{1}[j] f\right\|_{2}^{2}-\left\|\left(U_{1}[j] f\right) * r_{l, N-2}\right\|_{2}^{2}\right) \\
& =\left(\left\|f * g_{j}\right\|_{2}^{2}-\left\|\left|f * g_{j}\right| * r_{l, N-2}\right\|_{2}^{2}\right),
\end{aligned}
$$

where $r_{l, N-2}$ is the inverse Fourier transform of $\widehat{r}_{l}\left(\frac{\omega}{(5 / 3)^{N-2}}\right)$. Next, we note that $\widehat{r}_{l}\left(\frac{\omega}{(5 / 3)^{N-2}}\right)$ is a positive definite radial basis function [50, Theorem 6.20] and hence by [50, Theorem 6.18] $r_{l, N-2}(x) \geq 0$, for $x \in \mathbb{R}$. Furthermore, it follows from Lemma 3 in Appendix $\mathrm{C}$ that

$$
\left\|\left|f * g_{j}\right| * r_{l, N-2}\right\|_{2}^{2} \geq\left\|f * g_{j} *\left(M_{\nu_{j}} r_{l, N-2}\right)\right\|_{2}^{2},
$$

for $\left\{\nu_{j}\right\}_{j \in \mathbb{Z} \backslash\{0\}} \subseteq \mathbb{R}$. Choosing the modulation factors

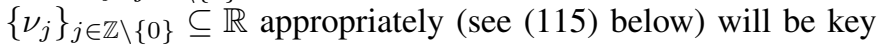
in establishing the inductive step. Using (110) and (111) to upper-bound the term $W_{N-1}\left(U_{1}[j] f\right)$ inside the sum on the RHS of 109 yields

$$
\begin{aligned}
W_{N}(f) & \leq \sum_{j \in \mathbb{Z} \backslash\{0\}}\left(\left\|f * g_{j}\right\|_{2}^{2}-\left\|f * g_{j} *\left(M_{\nu_{j}} r_{l, N-2}\right)\right\|_{2}^{2}\right) \\
& =\int_{\mathbb{R}}|\widehat{f}(\omega)|^{2} h_{l, N-2}(\omega) \mathrm{d} \omega
\end{aligned}
$$

where

$$
\begin{aligned}
& h_{l, N-2}(\omega) \\
& :=\sum_{j \in \mathbb{Z} \backslash\{0\}}\left|\widehat{g}_{j}(\omega)\right|^{2}\left(1-\left|\widehat{r}_{l}\left(\frac{\omega-\nu_{j}}{(5 / 3)^{N-2}}\right)\right|^{2}\right) .
\end{aligned}
$$

In (112) we employed Parseval's formula together with $\widehat{M_{\omega} f}=T_{\omega} \widehat{f}$, for $f \in L^{2}(\mathbb{R})$ and $\omega \in \mathbb{R}$. The key step is now to establish-by judiciously choosing $\left\{\nu_{j}\right\}_{j \in \mathbb{Z} \backslash\{0\}} \subseteq \mathbb{R}$ - the upper bound

$$
h_{l, N-2}(\omega) \leq\left(1-\left|\widehat{r}_{l}\left(\frac{\omega}{(5 / 3)^{N-1}}\right)\right|^{2}\right), \quad \forall \omega \in \mathbb{R}
$$

which then yields 27) and thereby completes the proof. To this end, we set $\eta:=\frac{4}{5}$,

$$
\nu_{j}:=2^{j} \eta, \quad j \geq 1, \quad \nu_{j}:=-2^{|j|} \eta, \quad j \leq-1,
$$

and note that it suffices to prove 114 for $\omega \geq 0$, as

$$
\begin{aligned}
& h_{l, N-2}(-\omega)=\sum_{j \in \mathbb{Z} \backslash\{0\}}\left|\widehat{g}_{j}(-\omega)\right|^{2}\left(1-\left|\widehat{r_{l}}\left(\frac{-\omega-\nu_{j}}{(5 / 3)^{N-2}}\right)\right|^{2}\right) \\
& =\sum_{j \leq-1}\left|\widehat{g_{j}}(-\omega)\right|^{2}\left(1-\left|\widehat{r_{l}}\left(\frac{\omega+\nu_{j}}{(5 / 3)^{N-2}}\right)\right|^{2}\right) \\
& =\sum_{j \geq 1}\left|\widehat{g_{-j}}(-\omega)\right|^{2}\left(1-\left|\widehat{r_{l}}\left(\frac{\omega+\nu_{-j}}{(5 / 3)^{N-2}}\right)\right|^{2}\right) \\
& =\sum_{j \geq 1}\left|\widehat{g_{j}}(\omega)\right|^{2}\left(1-\left|\widehat{r_{l}}\left(\frac{\omega-\nu_{j}}{(5 / 3)^{N-2}}\right)\right|^{2}\right) \\
& =h_{l, N-2}(\omega), \quad \forall \omega \geq 0 .
\end{aligned}
$$

Here, 116 is thanks to $\widehat{g_{j}}(-\omega)=0$, for $j \geq 1$ and $\omega \geq 0$, which is by 102, and 118) is owing to $\widehat{g_{j}}(\omega)=0$, for $j \leq-1$ and $\omega \geq 0$, which is by (103). Moreover, in (116) we used $\widehat{r}_{l}(-\omega)=\widehat{r}_{l}(\omega)$, for $\omega \in \mathbb{R}$, and (117) is thanks to

$$
\widehat{g_{-j}}(-\omega)=\widehat{\psi}\left(2^{-|-j|} \omega\right)=\widehat{\psi}\left(2^{-j} \omega\right)=\widehat{g_{j}}(\omega),
$$

for $\omega \in \mathbb{R}$ and $j \geq 1$, as well as

$$
\nu_{-j}=-2^{j} \eta=-\nu_{j}, \quad j \geq 1 .
$$

Now, let $\omega \in[0,1]$, and note that

$$
\begin{aligned}
h_{l, N-2}(\omega) & =\sum_{j \in \mathbb{Z} \backslash\{0\}}\left|\widehat{g}_{j}(\omega)\right|^{2}\left(1-\left|\widehat{r}_{l}\left(\frac{\omega-\nu_{j}}{(5 / 3)^{N-2}}\right)\right|^{2}\right)=0 \\
& \leq 1-\left|\widehat{r}_{l}\left(\frac{\omega}{(5 / 3)^{N-1}}\right)\right|^{2}, \quad \forall N \geq 2
\end{aligned}
$$

where the second equality in 119 is simply a consequence of $\widehat{g_{j}}(\omega)=0$, for $j \in \mathbb{Z} \backslash\{0\}$ and $\omega \in[0,1]$, which, in turn, is by (102) and (103). The inequality in 119 is thanks to $0 \leq \widehat{r}_{l}(\omega) \leq 1$, for $\omega \in \mathbb{R}$. Next, let $\omega \in[1,2]$. Then, we have

$$
\begin{aligned}
& h_{l, N-2}(\omega)=\left|\widehat{g_{1}}(\omega)\right|^{2}\left(1-\left|\widehat{r_{l}}\left(\frac{\omega-2 \eta}{(5 / 3)^{N-2}}\right)\right|^{2}\right) \\
& \leq\left|\widehat{g_{1}}(\omega)\right|^{2}\left(1-\left|\widehat{r}_{l}\left(\frac{\omega-2 \eta}{(5 / 3)^{N-2}}\right)\right|^{2}\right) \\
& +\underbrace{\left(1-\left|\widehat{g_{1}}(\omega)\right|^{2}\right)}_{\geq 0} \underbrace{\left(1-\left|\widehat{r_{l}}\left(\frac{\omega-\eta}{(5 / 3)^{N-2}}\right)\right|^{2}\right)}_{\geq 0} \\
& =1-\left|\widehat{r_{l}}\left(\frac{\omega-\eta}{(5 / 3)^{N-2}}\right)\right|^{2}+\left|\widehat{g_{1}}(\omega)\right|^{2}\left(\left|\widehat{r_{l}}\left(\frac{\omega-\eta}{(5 / 3)^{N-2}}\right)\right|^{2}\right. \\
& \left.-\left|\widehat{r_{l}}\left(\frac{\omega-2 \eta}{(5 / 3)^{N-2}}\right)\right|^{2}\right)
\end{aligned}
$$

where 120 is thanks to $\widehat{g_{j}}(\omega)=0$, for $j \in \mathbb{Z} \backslash\{0,1\}$ and $\omega \in[1,2]$, which, in turn, is by 102 and 103. Moreover, 121) is owing to

$$
\left|\widehat{g_{1}}(\omega)\right|^{2} \in[0,1],
$$




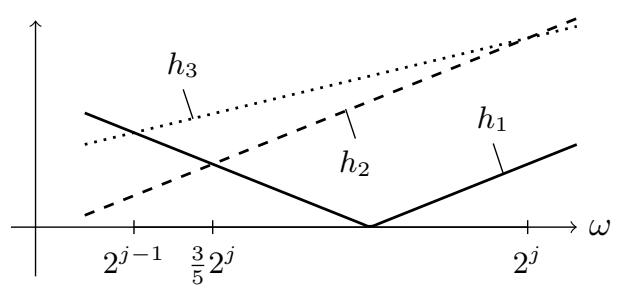

Fig. 10: The functions $h_{1}(\omega):=\left|\omega-2^{j} \eta\right|$ (solid line), $h_{2}(\omega):=\left|\omega-2^{j-1} \eta\right|$ (dashed line), and $h_{3}(\omega):=\frac{3}{5} \omega$ (dotted line) satisfy $h_{2} \leq h_{1} \leq h_{3}$ on $I_{L}=\left[2^{j-1}, \frac{3}{5} 2^{j}\right]$ and $h_{1} \leq h_{2} \leq h_{3}$ on $I_{R}=\left[\frac{3}{5} 2^{j}, 2^{j}\right]$.

which, in turn, is by 26 and $0 \leq \widehat{r}_{l}(\omega) \leq 1$, for $\omega \in \mathbb{R}$. Next, fix $j \geq 2$ and let $\omega \in\left[2^{j-1}, 2^{j}\right]$. Then, we have

$$
\begin{aligned}
& h_{l, N-2}(\omega)=\left|\widehat{g_{j}}(\omega)\right|^{2}\left(1-\left|\widehat{r_{l}}\left(\frac{\omega-2^{j} \eta}{(5 / 3)^{N-2}}\right)\right|^{2}\right) \\
& +\underbrace{\left|\widehat{g_{j-1}}(\omega)\right|^{2}}_{\geq 0} \underbrace{\left(1-\left|\widehat{g}_{j}(\omega)\right|^{2}-|\widehat{\phi}(\omega)|^{2}\right)} \underbrace{\left.\left.\left(1-\frac{\omega-2^{j-1} \eta}{(5 / 3)^{N-2}}\right)\right|^{2}\right)} \\
& \quad \leq 1-\left|\widehat{r_{l}}\left(\frac{\omega-2^{j-1} \eta}{(5 / 3)^{N-2}}\right)\right|^{2}+\left|\widehat{g}_{j}(\omega)\right|^{2}\left(\left|\widehat{r_{l}}\left(\frac{\omega-2^{j-1} \eta}{(5 / 3)^{N-2}}\right)\right|^{2}\right. \\
& \left.\left.\left(\frac{\omega-2^{j} \eta}{(5 / 3)^{N-2}}\right)\right|^{2}\right),
\end{aligned}
$$

where (123) is thanks to i) $\widehat{g_{j^{\prime}}}(\omega)=0$, for $j^{\prime} \in \mathbb{Z} \backslash\{0, j, j-1\}$ and $\omega \in\left[2^{j-1}, 2^{j}\right]$, which, in turn, is by (102) and (103), and ii)

$$
|\widehat{\phi}(\omega)|^{2}+\left|\widehat{g_{j-1}}(\omega)\right|^{2}+\left|\widehat{g_{j}}(\omega)\right|^{2}=1,
$$

for $\omega \in\left[2^{j-1}, 2^{j}\right]$, which is a consequence of the LittlewoodPaley condition (26) and of (102) and (103). It follows from (122) and (124) that for every $j \geq 1$, we have

$$
\begin{aligned}
& h_{l, N-2}(\omega) \leq 1-\left|\widehat{r}_{l}\left(\frac{\omega-2^{j-1} \eta}{(5 / 3)^{N-2}}\right)\right|^{2} \\
& +\left|\widehat{g}_{j}(\omega)\right|^{2} \underbrace{\left(\left.\widehat{r}_{l}\left(\frac{\omega-2^{j-1} \eta}{(5 / 3)^{N-2}}\right)\right|^{2}-\left|\widehat{r}_{l}\left(\frac{\omega-2^{j} \eta}{(5 / 3)^{N-2}}\right)\right|^{2}\right)}_{=: s(\omega)},
\end{aligned}
$$

for $\omega \in\left[2^{j-1}, 2^{j}\right]$. Next, we divide the interval $\left[2^{j-1}, 2^{j}\right]$ into two intervals, namely $I_{L}:=\left[2^{j-1}, \frac{3}{5} 2^{j}\right]$ and $I_{R}:=\left[\frac{3}{5} 2^{j}, 2^{j}\right]$, and note that $s(\omega) \geq 0$, for $\omega \in I_{L}$, and $s(\omega) \leq 0$, for $\omega \in I_{R}$, as $\widehat{r_{l}}$ is monotonically decreasing in $|\omega|$ and $\left|\omega-2^{j} \eta\right| \geq \mid \omega-$ $2^{j-1} \eta \mid$, for $\omega \in I_{L}$, and $\left|\omega-2^{j} \eta\right| \leq\left|\omega-2^{j-1} \eta\right|$, for $\omega \in I_{R}$, respectively (see Figure 10p. For $\omega \in I_{L}$, we therefore have

$$
\begin{aligned}
h_{l, N-2}(\omega) & \leq 1-\left|\widehat{r}_{l}\left(\frac{\omega-2^{j-1} \eta}{(5 / 3)^{N-2}}\right)\right|^{2}+\underbrace{\left|\widehat{g}_{j}(\omega)\right|^{2}}_{\in[0,1]} \underbrace{s(\omega)}_{\geq 0} \\
& \leq 1-\left|\widehat{r}_{l}\left(\frac{\omega-2^{j-1} \eta}{(5 / 3)^{N-2}}\right)\right|^{2}+s(\omega) \\
& =1-\left|\widehat{r}_{l}\left(\frac{\omega-2^{j} \eta}{(5 / 3)^{N-2}}\right)\right|^{2} \leq 1-\left|\widehat{r}_{l}\left(\frac{\omega}{(5 / 3)^{N-1}}\right)\right|^{2},
\end{aligned}
$$

where $\left|\widehat{g_{j}}(\omega)\right|^{2} \in[0,1]$ follows from $(125)$, and the last inequality is a consequence of $\left|\omega-2^{j} \eta\right| \leq \frac{3 \omega}{5}$, for $\omega \in I_{L}$, see Figure 10 For $\omega \in I_{R}$, we have

$$
\begin{aligned}
& h_{l, N-2}(\omega) \leq 1-\left|\widehat{r}_{l}\left(\frac{\omega-2^{j-1} \eta}{(5 / 3)^{N-2}}\right)\right|^{2}+\underbrace{\left|\widehat{g}_{j}(\omega)\right|^{2}}_{\in[0,1]} \underbrace{s(\omega)}_{\leq 0} \\
& \leq 1-\left|\widehat{r}_{l}\left(\frac{\omega-2^{j-1} \eta}{(5 / 3)^{N-2}}\right)\right|^{2} \leq 1-\left|\widehat{r}_{l}\left(\frac{\omega}{(5 / 3)^{N-1}}\right)\right|^{2},
\end{aligned}
$$

where the last inequality now follows from $\left|\omega-2^{j-1} \eta\right| \leq \frac{3 \omega}{5}$, for $\omega \in I_{R}$, see Figure 10] This completes the proof of (27).

Next, we establish (28). The proof is very similar to that of statement ii) in Theorem 2 in Section D We start by noting that (28) amounts to the existence of constants $C_{1, s}, C_{2, s}>0$ (that are independent of $N$ ) such that

$$
W_{N}(f) \leq C_{1, s}(5 / 3)^{-2 s N}, \quad \forall s \in(0,1 / 2), \forall N \geq 1,
$$

and

$$
W_{N}(f) \leq C_{2, s}(5 / 3)^{-N}, \quad \forall s \in[1 / 2, \infty), \forall N \geq 1 .
$$

The key idea of the proof of 126 is to upper-bound the integral on the RHS of 27] according to

$$
\begin{aligned}
& \int_{\mathbb{R}}|\widehat{f}(\omega)|^{2}\left(1-\left|\widehat{r_{l}}\left(\frac{\omega}{(5 / 3)^{N-1}}\right)\right|^{2}\right) \mathrm{d} \omega \\
& \leq \int_{\mathbb{R}}|\widehat{f}(\omega)|^{2} \min \left\{1, \frac{2 l|\omega|}{(5 / 3)^{N-1}}\right\} \mathrm{d} \omega \\
& =\int_{B_{\tau}(0)}|\widehat{f}(\omega)|^{2} \frac{2 l|\omega|}{(5 / 3)^{N-1}} \mathrm{~d} \omega+\int_{\mathbb{R} \backslash B_{\tau}(0)}|\widehat{f}(\omega)|^{2} \mathrm{~d} \omega,
\end{aligned}
$$

where $\tau:=\frac{(5 / 3)^{N-1}}{2 l}$. Here, the inequality in 128 follows from (87), and (129) is owing to

$$
\min \left\{1, \frac{2 l|\omega|}{(5 / 3)^{N-1}}\right\}= \begin{cases}\frac{2 l|\omega|}{(5 / 3)^{N-1}}, & |\omega| \leq \tau, \\ 1, & |\omega|>\tau .\end{cases}
$$

Now, the first integral in (129) satisfies

$$
\begin{aligned}
& \int_{B_{\tau}(0)}|\widehat{f}(\omega)|^{2} \frac{2 l|\omega|}{(5 / 3)^{N-1}} \mathrm{~d} \omega \\
& =\frac{2 l}{(5 / 3)^{N-1}} \int_{B_{\tau}(0)}|\widehat{f}(\omega)|^{2}|\omega|^{1-2 s}|\omega|^{2 s} \mathrm{~d} \omega \\
& \leq \frac{2 l \tau^{1-2 s}}{(5 / 3)^{N-1}} \int_{B_{\tau}(0)}|\widehat{f}(\omega)|^{2} \underbrace{|\omega|^{2 s}}_{\leq\left(1+|\omega|^{2}\right)^{s}} \mathrm{~d} \omega \\
& \leq\left(\frac{2 l}{(5 / 3)^{N-1}}\right)^{2 s} \int_{B_{\tau}(0)}|\widehat{f}(\omega)|^{2}\left(1+|\omega|^{2}\right)^{s} \mathrm{~d} \omega,
\end{aligned}
$$

where 130 is owing to $|\omega| \mapsto(1+|\omega|)^{1-2 s}$ monotonically increasing in $|\omega|$ for $s \in(0,1 / 2)$. For the second integral in 129, we have

$$
\begin{aligned}
& \int_{\mathbb{R} \backslash B_{\tau}(0)}|\widehat{f}(\omega)|^{2} \mathrm{~d} \omega=\int_{\mathbb{R} \backslash B_{\tau}(0)}|\widehat{f}(\omega)|^{2}|\omega|^{-2 s}|\omega|^{2 s} \mathrm{~d} \omega \\
& \leq \tau^{-2 s} \int_{\mathbb{R} \backslash B_{\tau}(0)}|\widehat{f}(\omega)|^{2} \underbrace{|\omega|^{2 s}}_{\leq\left(1+|\omega|^{2}\right)^{s}} \mathrm{~d} \omega \\
& \leq\left(\frac{2 l}{(5 / 3)^{N-1}}\right)^{2 s} \int_{\mathbb{R} \backslash B_{\tau}(0)}|\widehat{f}(\omega)|^{2}\left(1+|\omega|^{2}\right)^{s} \mathrm{~d} \omega,
\end{aligned}
$$

where (132) is thanks to $|\omega| \mapsto|\omega|^{-2 s}$ monotonically decreas- 
ing in $|\omega|$ for $s \in(0,1 / 2)$. Inserting (131) and (133) into (129) establishes 126 with

$$
C_{1, s}:=(2 l)^{2 s}(5 / 3)^{2 s}\|f\|_{H^{s}}^{2} .
$$

Next, we show (127) by noting that

$$
\begin{aligned}
& \int_{\mathbb{R}}|\widehat{f}(\omega)|^{2}\left(1-\left|\widehat{r_{l}}\left(\frac{\omega}{(5 / 3)^{N-1}}\right)\right|^{2}\right) \mathrm{d} \omega \\
& \leq \int_{\mathbb{R}}|\widehat{f}(\omega)|^{2} \min \left\{1, \frac{2 l|\omega|}{(5 / 3)^{N-1}}\right\} \mathrm{d} \omega \\
& \leq \frac{2 l}{(5 / 3)^{N-1}} \int_{\mathbb{R}}|\widehat{f}(\omega)|^{2}|\omega| \mathrm{d} \omega \\
& \leq \frac{2 l}{(5 / 3)^{N-1}} \int_{\mathbb{R}}|\widehat{f}(\omega)|^{2}\left(1+|\omega|^{2}\right)^{s} \mathrm{~d} \omega=\frac{2 l}{(5 / 3)^{N-1}}\|f\|_{H^{s}}^{2},
\end{aligned}
$$

where (134) is by (87), and the last inequality follows from

$$
|\omega| \leq\left(1+|\omega|^{2}\right)^{s}, \quad \forall \omega \in \mathbb{R}, \forall s \in[1 / 2, \infty) .
$$

This establishes (127) with

$$
C_{2, s}:=2 l(5 / 3)\|f\|_{H^{s}}^{2}
$$

and thereby completes the proof of statement i).

We proceed to the proof of statement ii), again, effected by induction over $N$. Specifically, we first establish (30) by employing the same arguments as those leading to 112 with $(5 / 3)^{N-2}$ replaced by $(3 / 2)^{N-2} R$. With this replacement $h_{l, N-2}$ in (113) becomes

$$
\begin{aligned}
& h_{l, N-2}(\omega) \\
& :=\sum_{k \in \mathbb{Z} \backslash\{0\}}\left|\widehat{g_{k}}(\omega)\right|^{2}\left(1-\left|\widehat{r}_{l}\left(\frac{\omega-\nu_{k}}{(3 / 2)^{N-2} R}\right)\right|^{2}\right),
\end{aligned}
$$

where, again, judicious choice of the modulation factors

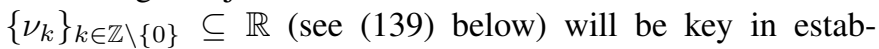
lishing the inductive step. Here, we note that the functions $\widehat{g_{k}}$ in 135 satisfy $\widehat{g_{k}}(\omega)=\widehat{g}(\omega-R(k+1))$, for $k \geq 1$, $\widehat{g_{k}}(\omega)=\widehat{g}(\omega+R(|k|+1))$, for $k \leq-1$, both by assumption, as well as

$$
\begin{aligned}
\operatorname{supp}\left(\widehat{g_{k}}\right) & =\operatorname{supp}(\widehat{g}(\cdot-R(k+1))) \\
& \subseteq[R k, R(k+2)], \quad k \geq 1,
\end{aligned}
$$

and

$$
\begin{aligned}
\operatorname{supp}\left(\widehat{g_{k}}\right) & =\operatorname{supp}(\widehat{g}(\cdot+R(|k|+1))) \\
& \subseteq[-R(|k|+2),-R|k|], \quad k \leq-1,
\end{aligned}
$$

where (136) and (137) follow from

$$
\operatorname{supp}(\widehat{g}) \subseteq[-R, R]
$$

which again is by assumption. It remains to establish the equivalent of (114), namely

$$
h_{l, N-2}(\omega) \leq 1-\left|\widehat{r}_{l}\left(\frac{\omega}{(3 / 2)^{N-1} R}\right)\right|^{2}, \quad \forall \omega \in \mathbb{R} .
$$

To this end, we set $\eta:=\frac{2}{3} R$,

$$
\nu_{k}:=R k+\eta, \quad \forall k \geq 1, \quad \nu_{k}:=-\nu_{|k|}, \quad \forall k \leq-1,
$$

and note that it suffices to establish (138) for $\omega \geq 0$, thanks to

$$
\begin{aligned}
& h_{l, N-2}(-\omega)=\sum_{k \in \mathbb{Z} \backslash\{0\}}\left|\widehat{g}_{k}(-\omega)\right|^{2}\left(1-\left|\widehat{r_{l}}\left(\frac{-\omega-\nu_{k}}{(3 / 2)^{N-2} R}\right)\right|^{2}\right) \\
& =\sum_{k \leq-1}\left|\widehat{g_{k}}(-\omega)\right|^{2}\left(1-\left|\widehat{r_{l}}\left(\frac{\omega+\nu_{k}}{(3 / 2)^{N-2} R}\right)\right|^{2}\right) \\
& =\sum_{k \geq 1}\left|\widehat{g_{-k}}(-\omega)\right|^{2}\left(1-\left|\widehat{r_{l}}\left(\frac{\omega+\nu_{-k}}{(3 / 2)^{N-2} R}\right)\right|^{2}\right) \\
& =\sum_{k \geq 1}\left|\widehat{g_{k}}(\omega)\right|^{2}\left(1-\left|\widehat{r_{l}}\left(\frac{\omega-\nu_{k}}{(3 / 2)^{N-2} R}\right)\right|^{2}\right) \\
& =h_{l, N-2}(\omega), \quad \forall \omega \geq 0 .
\end{aligned}
$$

Here, (140) follows from $\widehat{g_{k}}(-\omega)=0$, for $k \geq 1$ and $\omega \geq 0$, which, in turn, is by (136), and (142) is owing to $\widehat{g_{k}}(\omega)=0$, for $k \leq-1$ and $\omega \geq 0$, which is by (137). Moreover, in (140) we used $\widehat{r}_{l}(-\omega)=\widehat{r_{l}}(\omega)$, for $\omega \in \mathbb{R}$, and (141) is thanks to $\nu_{-k}=-\nu_{k}$, for $k \geq 1$, and

$$
\begin{aligned}
\widehat{g_{-k}}(-\omega) & =\widehat{g}(-\omega+R(|-k|+1))=\widehat{g}(-(\omega-R(k+1))) \\
& =\widehat{g}(\omega-R(k+1))=\widehat{g_{k}}(\omega), \quad \forall \omega \in \mathbb{R}, \quad \forall k \geq 1,
\end{aligned}
$$

where we used $\widehat{g}(-\omega)=\widehat{g}(\omega)$, for $\omega \in \mathbb{R}$, which is by assumption. Now, let $\omega \in[0, R]$, and note that

$$
h_{l, N-2}(\omega)=0 \leq 1-\left|\widehat{r}_{l}\left(\frac{\omega}{(3 / 2)^{N-1} R}\right)\right|^{2},
$$

for $N \geq 2$, where the equality in 143 is a consequence of (136) and 137, and the inequality is thanks to $0 \leq \widehat{r}_{l}(\omega) \leq 1$, for $\omega \in \mathbb{R}$. Next, let $\omega \in[R, 2 R]$. Then, we have

$$
\begin{aligned}
& h_{l, N-2}(\omega)=\left|\widehat{g_{1}}(\omega)\right|^{2}\left(1-\left|\widehat{r_{l}}\left(\frac{\omega-\nu_{1}}{(3 / 2)^{N-2} R}\right)\right|^{2}\right) \\
& \leq\left|\widehat{g_{1}}(\omega)\right|^{2}\left(1-\mid \widehat{r_{l}}\left(\frac{\omega-\nu_{1}}{\left.\left.(3 / 2)^{N-2}\right)\left.\right|^{2}\right)}\right.\right. \\
& +\underbrace{\left(1-\left|\widehat{g}_{1}(\omega)\right|^{2}\right)}_{\geq 0} \underbrace{\left(1-\left|\widehat{r_{l}}\left(\frac{\omega-\eta}{(3 / 2)^{N-2} R}\right)\right|^{2}\right)}_{\geq 0} \\
& =1-\left|\widehat{r_{l}}\left(\frac{\omega-\eta}{(3 / 2)^{N-2} R}\right)\right|^{2}+\left|\widehat{g_{1}}(\omega)\right|^{2}\left(\left|\widehat{r}_{l}\left(\frac{\omega-\eta}{(3 / 2)^{N-2} R}\right)\right|^{2}\right. \\
& \left.-\left|\widehat{r_{l}}\left(\frac{\omega-\nu_{1}}{(3 / 2)^{N-2}}\right)\right|^{2}\right),
\end{aligned}
$$

where (144) is thanks to $\widehat{g_{k}}(\omega)=0$, for $k \in \mathbb{Z} \backslash\{0,1\}$ and $\omega \in[0, R]$, which, in turn, is by (136) and (137). Moreover, (145) is owing to $\left|\widehat{g_{1}}(\omega)\right|^{2} \in[0,1]$, which, in turn, is by [29], and $0 \leq \widehat{r}_{l}(\omega) \leq 1$, for $\omega \in \mathbb{R}$. Next, fix $k \geq 2$, and let $\omega \in[R k, R(k+1)]$. Then, we have

$$
\begin{aligned}
& h_{l, N-2}(\omega)=\left|\widehat{g_{k}}(\omega)\right|^{2}\left(1-\left|\widehat{r_{l}}\left(\frac{\omega-\nu_{k}}{(3 / 2)^{N-2} R}\right)\right|^{2}\right) \\
& +\underbrace{\left|\widehat{g_{k-1}}(\omega)\right|^{2}}_{=\left(1-\left|\widehat{g_{k}}(\omega)\right|^{2}-|\widehat{\phi}(\omega)|^{2}\right)} \underbrace{\left(1-\left|\widehat{r_{l}}\left(\frac{\omega-\nu_{k-1}}{(3 / 2)^{N-2} R}\right)\right|^{2}\right)}_{\geq 0} \\
& \leq 1-\left|\widehat{r_{l}}\left(\frac{\omega-\nu_{k-1}}{(3 / 2)^{N-2} R}\right)\right|^{2}+\left|\widehat{g_{k}}(\omega)\right|^{2}\left(\left|\widehat{r_{l}}\left(\frac{\omega-\nu_{k-1}}{(3 / 2)^{N-2} R}\right)\right|^{2}\right. \\
& \left.-\left|\widehat{r_{l}}\left(\frac{\omega-\nu_{k}}{\left.(3 / 2)^{N-2}\right)}\right)\right|^{2}\right),
\end{aligned}
$$




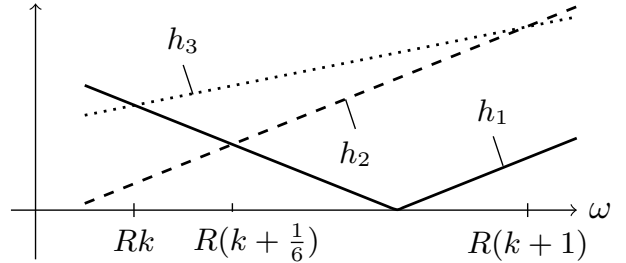

Fig. 11: The functions $h_{1}(\omega):=\left|\omega-\nu_{k}\right|$ (solid line), $h_{2}(\omega):=\left|\omega-\nu_{k-1}\right|$ (dashed line), and $h_{3}(\omega)=\frac{2}{3} \omega$ (dotted line) satisfy $h_{2} \leq h_{1} \leq h_{3}$ on $I_{L}=\left[R k, R\left(k+\frac{1}{6}\right)\right]$ and $h_{1} \leq h_{2} \leq h_{3}$ on $I_{R}=\left[R\left(k+\frac{1}{6}\right), R(k+1)\right]$.

where (147) is thanks to i) $\widehat{g_{k^{\prime}}}(\omega)=0$, for $k^{\prime} \in \mathbb{Z} \backslash\{0, k, k-1\}$ and $\omega \in[R k, R(k+1)]$, which, in turn, is by [136) and [137], and ii)

$$
|\widehat{\chi}(\omega)|^{2}+\left|\widehat{g_{k-1}}(\omega)\right|^{2}+\left|\widehat{g_{k}}(\omega)\right|^{2}=1,
$$

for $\omega \in[R k, R(k+1)]$, which is a consequence of the Littlewood-Paley condition (29) and of (136) and (137). It follows from (146) and (148) that for $k \geq 1$,

$$
\begin{aligned}
& h_{l, N-2}(\omega) \leq 1-\left|\widehat{r}_{l}\left(\frac{\omega-\nu_{k-1}}{(3 / 2)^{N-2} R}\right)\right|^{2} \\
& +\left|\widehat{g}_{k}(\omega)\right|^{2} \underbrace{\left(\left|\widehat{r_{l}}\left(\frac{\omega-\nu_{k-1}}{(3 / 2)^{N-2}}\right)\right|^{2}-\left|\widehat{r_{l}}\left(\frac{\omega-\nu_{k}}{(3 / 2)^{N-2} R}\right)\right|^{2}\right)}_{=: s(\omega)},
\end{aligned}
$$

for $\omega \in[R k, R(k+1)]$, where $\nu_{0}:=\eta$. Next, we divide the interval $[R k, R(k+1)]$ into two intervals, namely

$$
I_{L}:=[R k, R(k+1 / 6)]
$$

and

$$
I_{R}:=[R(k+1 / 6), R(k+1)],
$$

and note that $s(\omega) \geq 0$, for $\omega \in I_{L}$, and $s(\omega) \leq 0$, for $\omega \in I_{R}$, as $\widehat{r_{l}}$ is monotonically decreasing in $|\omega|$ and $\left|\omega-\nu_{k}\right| \geq$ $\left|\omega-\nu_{k-1}\right|$, for $\omega \in I_{L}$, and $\left|\omega-\nu_{k}\right| \leq\left|\omega-\nu_{k-1}\right|$, for $\omega \in I_{R}$, respectively (see Figure 11. For $\omega \in I_{L}$, we therefore have

$$
\begin{aligned}
& h_{l, N-2}(\omega) \leq 1-\left|\widehat{r}_{l}\left(\frac{\omega-\nu_{k-1}}{(3 / 2)^{N-2} R}\right)\right|^{2}+\underbrace{\left|\widehat{g}_{k}(\omega)\right|^{2}}_{\in[0,1]} \underbrace{s(\omega)}_{\geq 0} \\
& \leq 1-\left|\widehat{r}_{l}\left(\frac{\omega-\nu_{k-1}}{(3 / 2)^{N-2} R}\right)\right|^{2}+s(\omega) \\
& =1-\left|\widehat{r}_{l}\left(\frac{\omega-\nu_{k}}{(3 / 2)^{N-2} R}\right)\right|^{2} \leq 1-\left|\widehat{r}_{l}\left(\frac{\omega}{(3 / 2)^{N-1} R}\right)\right|^{2},
\end{aligned}
$$

where $\left|\widehat{g_{k}}(\omega)\right|^{2} \in[0,1]$ follows from (149), and the last inequality is by $\left|\omega-\nu_{k}\right| \leq \frac{2 \omega}{3}$, for $\omega \in I_{L}$ (see Figure 11 . For the interval $\omega \in I_{R}$, we have

$$
\begin{aligned}
& h_{l, N-2}(\omega) \leq 1-\left|\widehat{r}_{l}\left(\frac{\omega-\nu_{k-1}}{(3 / 2)^{N-2} R}\right)\right|^{2}+\underbrace{\left|\widehat{g}_{k}(\omega)\right|^{2}}_{\in[0,1]} \underbrace{s(\omega)}_{\leq 0} \\
& \leq 1-\left|\widehat{r}_{l}\left(\frac{\omega-\nu_{k-1}}{(3 / 2)^{N-2} R}\right)\right|^{2} \leq 1-\left|\widehat{r}_{l}\left(\frac{\omega}{(3 / 2)^{N-1} R}\right)\right|^{2},
\end{aligned}
$$

where the last inequality is by $\left|\omega-\nu_{k-1}\right| \leq \frac{2 \omega}{3}$, for $\omega \in I_{R}$ (see Figure 11. This completes the proof of 30.

Next, we establish (31). The proof is very similar to that of statement ii) in Theorem 1 in Appendix $\mathrm{D}$. We start by noting that (31) amounts to the existence of constants $C_{1, s}, C_{2, s}>0$ (that are independent of $N$ ) such that

$$
W_{N}(f) \leq C_{1, s}(3 / 2)^{-2 s N}, \quad \forall s \in(0,1 / 2), \forall N \geq 1,
$$

and

$$
W_{N}(f) \leq C_{2, s}(3 / 2)^{-N}, \quad \forall s \in[1 / 2, \infty), \forall N \geq 1 .
$$

The key idea of the proof of 150 is to upper-bound the integral on the RHS of (30) according to

$$
\begin{aligned}
& \int_{\mathbb{R}}|\widehat{f}(\omega)|^{2}\left(1-\left|\widehat{r_{l}}\left(\frac{\omega}{(3 / 2)^{N-1} R}\right)\right|^{2}\right) \mathrm{d} \omega \\
& \leq \int_{\mathbb{R}}|\widehat{f}(\omega)|^{2} \min \left\{1, \frac{2 l|\omega|}{(3 / 2)^{N-1} R}\right\} \mathrm{d} \omega \\
& =\int_{B_{\tau}(0)}|\widehat{f}(\omega)|^{2} \frac{2 l|\omega|}{(3 / 2)^{N-1} R} \mathrm{~d} \omega+\int_{\mathbb{R} \backslash B_{\tau}(0)}|\widehat{f}(\omega)|^{2} \mathrm{~d} \omega,
\end{aligned}
$$

where

$$
\tau:=\frac{(3 / 2)^{N-1} R}{2 l} .
$$

Here, the inequality in 152 follows from $(87)$, and $(153)$ is owing to

$$
\min \left\{1, \frac{2 l|\omega|}{(3 / 2)^{N-1} R}\right\}= \begin{cases}\frac{2 l|\omega|}{(3 / 2)^{N-1} R}, & |\omega| \leq \tau, \\ 1, & |\omega|>\tau .\end{cases}
$$

Now, the first integral in 153 satisfies

$$
\begin{aligned}
& \int_{B_{\tau}(0)}|\widehat{f}(\omega)|^{2} \frac{2 l|\omega|}{(3 / 2)^{N-1} R} \mathrm{~d} \omega \\
& =\frac{2 l}{(3 / 2)^{N-1} R} \int_{B_{\tau}(0)}|\widehat{f}(\omega)|^{2}|\omega|^{1-2 s}|\omega|^{2 s} \mathrm{~d} \omega \\
& \leq \frac{2 l \tau^{1-2 s}}{(3 / 2)^{N-1} R} \int_{B_{\tau}(0)}|\widehat{f}(\omega)|^{2} \underbrace{|\omega|^{2 s}}_{\leq\left(1+|\omega|^{2}\right)^{s}} \mathrm{~d} \omega \\
& \leq\left(\frac{2 l}{(3 / 2)^{N-1} R}\right)^{2 s} \int_{B_{\tau}(0)}|\widehat{f}(\omega)|^{2}\left(1+|\omega|^{2}\right)^{s} \mathrm{~d} \omega,
\end{aligned}
$$

where (154) is owing to

$$
|\omega| \mapsto(1+|\omega|)^{1-2 s}
$$

monotonically increasing in $|\omega|$ for $s \in(0,1 / 2)$. For the second integral in (153), we have

$$
\begin{aligned}
& \int_{\mathbb{R} \backslash B_{\tau}(0)}|\widehat{f}(\omega)|^{2} \mathrm{~d} \omega=\int_{\mathbb{R} \backslash B_{\tau}(0)}|\widehat{f}(\omega)|^{2}|\omega|^{-2 s}|\omega|^{2 s} \mathrm{~d} \omega \\
& \leq \tau^{-2 s} \int_{\mathbb{R} \backslash B_{\tau}(0)}|\widehat{f}(\omega)|^{2} \underbrace{\mid \omega s}_{\leq\left(1+|\omega|^{2}\right)^{s}} \mathrm{~d} \omega \\
& \leq\left(\frac{2 l}{(3 / 2)^{N-1} R}\right)^{2 s} \int_{\mathbb{R} \backslash B_{\tau}(0)}|\widehat{f}(\omega)|^{2}\left(1+|\omega|^{2}\right)^{s} \mathrm{~d} \omega,
\end{aligned}
$$

where (156) is thanks to

$$
|\omega| \mapsto|\omega|^{-2 s}, \quad \omega \in \mathbb{R}^{d},
$$

monotonically decreasing in $|\omega|$ for $s \in(0,1 / 2)$. Inserting (155) and 157) into (153) establishes (150) with

$$
C_{1, s}:=(2 l)^{2 s}(3 / 2)^{2 s} R^{-2 s}\|f\|_{H^{s}}^{2} .
$$


Next, we show (151) by noting that

$$
\begin{aligned}
& \int_{\mathbb{R}}|\widehat{f}(\omega)|^{2}\left(1-\left|\widehat{r}_{l}\left(\frac{\omega}{(3 / 2)^{N-1} R}\right)\right|^{2}\right) \mathrm{d} \omega \\
& \leq \int_{\mathbb{R}}|\widehat{f}(\omega)|^{2} \min \left\{1, \frac{2 l|\omega|}{(3 / 2)^{N-1} R}\right\} \mathrm{d} \omega \\
& \leq \frac{2 l}{(3 / 2)^{N-1} R} \int_{\mathbb{R}}|\widehat{f}(\omega)|^{2}|\omega| \mathrm{d} \omega \\
& \leq \frac{2 l}{(3 / 2)^{N-1} R} \int_{\mathbb{R}}|\widehat{f}(\omega)|^{2}\left(1+|\omega|^{2}\right)^{s} \mathrm{~d} \omega \\
& =\frac{2 l}{(3 / 2)^{N-1} R}\|f\|_{H^{s}}^{2},
\end{aligned}
$$

where $(158)$ is by $(87)$, and the last inequality follows from $|\omega| \leq\left(1+|\omega|^{2}\right)^{s}$, for $\omega \in \mathbb{R}$ and $s \in[1 / 2, \infty)$. This establishes (151) with $C_{2, s}:=2 l(3 / 2) R^{-1}\|f\|_{H^{s}}^{2}$ and thereby completes the proof of statement ii).

\section{APPENDIX H}

Proof of Corollary 1

We start with statement i) and note that $A_{\Omega}^{N}=B_{\Omega}^{N}=1$, $N \in \mathbb{N}$, by assumption. Let $f \in L^{2}\left(\mathbb{R}^{d}\right)$ with

$$
\operatorname{supp}(\widehat{f}) \subseteq B_{L}(0) .
$$

Then, by Proposition 1 in Appendix E together with

$$
\lim _{N \rightarrow \infty} W_{N}(f)=0, \quad \forall f \in L^{2}\left(\mathbb{R}^{d}\right),
$$

which follows from Proposition 2 in Appendix F, we have

$$
\begin{aligned}
\|f\|_{2}^{2} & =\left|\left\|\Phi_{\Omega}(f)||^{2}=\left.\sum_{n=0}^{\infty}\left|\| \Phi_{\Omega}^{n}(f)\right|\right|^{2}\right.\right. \\
& \geq \sum_{n=0}^{N}\left\||| \Phi_{\Omega}^{n}(f)||^{2}=\right\| f \|_{2}^{2}-W_{N+1}(f) \\
& \geq \int_{\mathbb{R}^{d}}|\widehat{f}(\omega)|^{2}\left|\widehat{r}_{l}\left(\frac{\omega}{(N+1)^{\alpha} \delta}\right)\right|^{2} \mathrm{~d} \omega \\
& =\int_{B_{L}(0)}|\widehat{f}(\omega)|^{2}\left|\widehat{r}_{l}\left(\frac{\omega}{(N+1)^{\alpha} \delta}\right)\right|^{2} \mathrm{~d} \omega,
\end{aligned}
$$

where $(159)$ is by the lower bound in $(95,, 160)$ is thanks to Parseval's formula and (20), and (161) follows from $f$ being $L$-band-limited. Next, thanks to $\widehat{r_{l}}$ monotonically decreasing in $|\omega|$, we get

$$
\left|\widehat{r}_{l}\left(\frac{\omega}{(N+1)^{\alpha} \delta}\right)\right|^{2} \geq\left|\widehat{r}_{l}\left(\frac{L}{(N+1)^{\alpha} \delta}\right)\right|^{2},
$$

for $\omega \in B_{L}(0)$. Employing 162 in (161), we obtain

$$
\begin{aligned}
\|f\|_{2}^{2} & \geq\left|\widehat{r}_{l}\left(\frac{L}{(N+1)^{\alpha} \delta}\right)\right|^{2}\|f\|_{2}^{2} \\
& =\left(1-\frac{L}{(N+1)^{\alpha} \delta}\right)_{+}^{2 l}\|f\|_{2}^{2} \\
& =\left(1-\frac{L}{(N+1)^{\alpha} \delta}\right)^{2 l}\|f\|_{2}^{2} \geq(1-\varepsilon)\|f\|_{2}^{2},
\end{aligned}
$$

where in (163) we used Parseval's formula, the equality in (164) is due to $L \leq(N+1)^{\alpha} \delta$, which, in turn, is by (33), and the inequality in (164) is also by (33) (upon rearranging terms). This establishes (32) and thereby completes the proof.
The proof of statement ii) is very similar to that of statement i). Again, we start by noting that $A_{\Omega}^{N}=B_{\Omega}^{N}=1, N \in \mathbb{N}$, by assumption. Let $f \in L^{2}(\mathbb{R})$ with $\operatorname{supp}(\widehat{f}) \subseteq B_{L}(0)$. Then, by Proposition 1 in Appendix E together with $\lim _{N \rightarrow \infty} W_{N}(f)=0$, for $f \in L^{2}(\mathbb{R})$, we have

$$
\begin{aligned}
\|f\|_{2}^{2} & =\left\|\left|\Phi _ { \Omega } ( f ) \left\|\left.\right|^{2}=\sum_{n=0}^{\infty}\left|\left\|\Phi_{\Omega}^{n}(f)\right\|\right|^{2}\right.\right.\right. \\
& \geq \sum_{n=0}^{N}\left\||| \Phi_{\Omega}^{n}(f)||^{2}=\right\| f \|_{2}^{2}-W_{N+1}(f) \\
& \geq \int_{\mathbb{R}}|\widehat{f}(\omega)|^{2}\left|\widehat{r_{l}}\left(\frac{\omega}{a^{N} \delta}\right)\right|^{2} \mathrm{~d} \omega \\
& =\int_{B_{L}(0)}|\widehat{f}(\omega)|^{2}\left|\widehat{r}_{l}\left(\frac{\omega}{a^{N} \delta}\right)\right|^{2} \mathrm{~d} \omega,
\end{aligned}
$$

where (165) is by the lower bound in (95), (166) is thanks to Parseval's formula and (27) and (30), and (167) follows from $f$ being $L$-band-limited. Next, thanks to $\widehat{r_{l}}$ monotonically decreasing in $|\omega|$, we get

$$
\left|\widehat{r}_{l}\left(\frac{\omega}{a^{N} \delta}\right)\right|^{2} \geq\left|\widehat{r}_{l}\left(\frac{L}{a^{N} \delta}\right)\right|^{2}, \quad \forall \omega \in B_{L}(0) .
$$

Employing (168) in (167) yields

$$
\begin{aligned}
\|f\|_{2}^{2} & \geq\left|\widehat{r}_{l}\left(\frac{L}{a^{N} \delta}\right)\right|^{2}\|f\|_{2}^{2}=\left(1-\frac{L}{a^{N} \delta}\right)_{+}^{2 l}\|f\|_{2}^{2} \\
& =\left(1-\frac{L}{a^{N} \delta}\right)^{2 l}\|f\|_{2}^{2} \geq(1-\varepsilon)\|f\|_{2}^{2},
\end{aligned}
$$

where in (169) we used Parseval's formula, the equality in (170) is by $L \leq a^{N} \delta$, which, in turn, is by (34), and the inequality in (170) is also due to 34) (upon rearranging terms). This establishes (32) and thereby completes the proof of ii).

\section{APPENDIX I \\ PROOF OF COROLLARY 2}

The proof is very similar to that of Corollary 1 in Appendix $\mathrm{H}$. We start with statement i). Let $f \in H^{s}\left(\mathbb{R}^{d}\right) \backslash\{0\}$ and $\varepsilon \in$ $(0,1)$ and note that, by $(84)$ and $(85)$ together with $B_{\Omega}^{N}=1$, $N \in \mathbb{N}$, which is by assumption, we have

$$
W_{N}(f) \leq \frac{(2 l)^{\gamma}\|f\|_{H^{s}}^{2}}{\delta^{\gamma} N^{\gamma \alpha}}, \quad \forall s>0,
$$

where $\gamma=\min \{1,2 s\}$. By Proposition 1 in Appendix $\mathrm{E}$ with $A_{\Omega}^{N}=B_{\Omega}^{N}=1, N \in \mathbb{N}$, and $\lim _{N \rightarrow \infty} W_{N}(f)=0, f \in L^{2}\left(\mathbb{R}^{d}\right)$, which follows from Proposition 2 in Appendix F, we have

$$
\begin{aligned}
\|f\|_{2}^{2} & =\left.\left\|\left|\Phi_{\Omega}(f)\left\|\left.\right|^{2}=\sum_{n=0}^{\infty}\right\|\right| \Phi_{\Omega}^{n}(f)\right\|\right|^{2} \\
& \geq \sum_{n=0}^{N}\left\|\left|\Phi_{\Omega}^{n}(f)\left\|\left.\right|^{2}=\right\| f \|_{2}^{2}-W_{N+1}(f)\right.\right. \\
& \geq\|f\|_{2}^{2}-\frac{(2 l)^{\gamma}\|f\|_{H^{s}}^{2}}{\delta^{\gamma}(N+1)^{\gamma \alpha}} \\
& \geq\|f\|_{2}^{2}-\varepsilon\|f\|_{2}^{2}=(1-\varepsilon)\|f\|_{2}^{2},
\end{aligned}
$$

where (172) is by the lower bound in (95), (173) is thanks to (171), and (174) follows from (35). This establishes (32) and thereby completes the proof of i). 
The proof of statement ii) is very similar to that of statement i). Let $f \in H^{s}(\mathbb{R}) \backslash\{0\}$ and $\varepsilon \in(0,1)$ and note that, by $[126$, (127), (150), and (151), we have

$$
W_{N}(f) \leq \frac{(2 l)^{\gamma}\|f\|_{H^{s}}^{2}}{\delta^{\gamma} a^{\gamma(N-1)}}, \quad \forall s>0,
$$

where $\gamma=\min \{1,2 s\}$. By Proposition 1 in Appendix E with $A_{\Omega}^{N}=B_{\Omega}^{N}=1, N \in \mathbb{N}$, and $\lim _{N \rightarrow \infty} W_{N}(f)=0, f \in L^{2}(\mathbb{R})$, which follows from Proposition $\overrightarrow{2}$ in Appendix $\mathrm{F}$, we have

$$
\begin{aligned}
\|f\|_{2}^{2} & =\left.\left\||| \Phi_{\Omega}(f)\right\|\right|^{2}=\sum_{n=0}^{\infty}\left\|\mid \Phi_{\Omega}^{n}(f)\right\| \|^{2} \\
& \geq \sum_{n=0}^{N}\|\| \Phi_{\Omega}^{n}(f)\|\|^{2}=\|f\|_{2}^{2}-W_{N+1}(f) \\
& \geq\|f\|_{2}^{2}-\frac{(2 l)^{\gamma}\|f\|_{H^{s}}^{2}}{\delta^{\gamma} a^{\gamma N}} \\
& \geq\|f\|_{2}^{2}-\varepsilon\|f\|_{2}^{2}=(1-\varepsilon)\|f\|_{2}^{2},
\end{aligned}
$$

where $(176)$ is by the lower bound in 95 ,, 177$)$ is thanks to (175), and (178) follows from (36). This establishes (32) and thereby completes the proof of ii).

\section{ACKNOWLEDGMENTS}

The authors are thankful to Verner Vlačić for pointing out errors in arguments on the spectral decay of Sobolev functions and on the volume of tubes in an earlier version of the manuscript.

\section{REFERENCES}

[1] Y. LeCun, Y. Bengio, and G. Hinton, "Deep learning," Nature, vol. 521, pp. 436-444, 2015.

[2] I. Goodfellow, Y. Bengio, and A. Courville, Deep Learning. MIT Press, 2016. http://www.deeplearningbook.org

[3] Y. Bengio, A. Courville, and P. Vincent, "Representation learning: A review and new perspectives," IEEE Trans. Pattern Anal. Mach. Intell., vol. 35, no. 8, pp. 1798-1828, 2013.

[4] Y. LeCun, B. Boser, J. Denker, D. Henderson, R. Howard, W. Hubbard, and L. Jackel, "Handwritten digit recognition with a back-propagation network," in Proc. of International Conference on Neural Information Processing Systems (NIPS), pp. 396-404, 1990.

[5] D. E. Rumelhart, G. Hinton, and R. J. Williams, "Learning internal representations by error propagation," in Parallel distributed processing: Explorations in the microstructure of cognition (J. L. McClelland and D. E. Rumelhart, eds.), pp. 318-362, MIT Press, 1986.

[6] Y. LeCun, L. Bottou, Y. Bengio, and P. Haffner, "Gradient-based learning applied to document recognition," in Proc. of the IEEE, pp. 2278-2324, 1998.

[7] K. He, X. Zhang, S. Ren, and J. Sun, "Deep residual learning for image recognition," in Proc. of IEEE International Conference on Computer Vision and Pattern Recognition (CVPR), pp. 770-778, 2015.

[8] S. Mallat, "Group invariant scattering," Comm. Pure Appl. Math., vol. 65, no. 10, pp. 1331-1398, 2012.

[9] T. Wiatowski and H. Bölcskei, "A mathematical theory of deep convolutional neural networks for feature extraction," IEEE Trans. Inf. Theory, 2018, to appear.

[10] I. Waldspurger, Wavelet transform modulus: Phase retrieval and scattering. $\mathrm{PhD}$ thesis, École Normale Supérieure, Paris, 2015.

[11] W. Czaja and W. Li, "Analysis of time-frequency scattering transforms," arXiv:1606.08677, 2017.

[12] J. Bruna and S. Mallat, "Invariant scattering convolution networks," IEEE Trans. Pattern Anal. Mach. Intell., vol. 35, no. 8, pp. 1872-1886, 2013.

[13] J. Andén and S. Mallat, "Deep scattering spectrum," IEEE Trans. Signal Process., vol. 62, no. 16, pp. 4114-4128, 2014.

[14] L. Sifre, Rigid-motion scattering for texture classification. $\mathrm{PhD}$ thesis, Centre de Mathématiques Appliquées, École Polytechnique Paris-Saclay, 2014.
[15] T. Wiatowski, M. Tschannen, A. Stanić, P. Grohs, and H. Bölcskei, "Discrete deep feature extraction: A theory and new architectures," in Proc. of International Conference on Machine Learning (ICML), pp. 2149-2158, 2016.

[16] M. Tschannen, T. Kramer, G. Marti, M. Heinzmann, and T. Wiatowski, "Heart sound classification using deep structured features," in Proc. of Computing in Cardiology (CinC), pp. 565-568, 2016.

[17] M. Tschannen, L. Cavigelli, F. Mentzer, T. Wiatowski, and L. Benini, "Deep structured features for semantic segmentation," in Proc. of European Signal Processing Conference (EUSIPCO), pp. 61-65, 2017.

[18] R. Balan, M. Singh, and D. Zou, "Lipschitz properties for deep convolutional networks," arXiv:1701.05217, 2017.

[19] P. Grohs, T. Wiatowski, and H. Bölcskei, "Deep convolutional neural networks on cartoon functions," in Proc. of IEEE International Symposium on Information Theory (ISIT), pp. 1163-1167, 2016.

[20] C. M. Bishop, Pattern recognition and machine learning. Springer, 2009.

[21] K. Gröchenig, Foundations of time-frequency analysis. Birkhäuser, 2001.

[22] I. Daubechies, Ten lectures on wavelets. Society for Industrial and Applied Mathematics, 1992.

[23] S. Mallat, A wavelet tour of signal processing: The sparse way. Academic Press, 3rd ed., 2009.

[24]E. J. Candès and D. L. Donoho, "New tight frames of curvelets and optimal representations of objects with piecewise $C^{2}$ singularities," Comm. Pure Appl. Math., vol. 57, no. 2, pp. 219-266, 2004.

[25]E. J. Candès and D. L. Donoho, "Continuous curvelet transform: II. Discretization and frames," Appl. Comput. Harmon. Anal., vol. 19, no. 2, pp. 198-222, 2005.

[26] P. Grohs, S. Keiper, G. Kutyniok, and M. Schäfer, "Cartoon approximation with $\alpha$-curvelets," J. Fourier Anal. Appl., pp. 1-59, 2015.

[27] K. Guo, G. Kutyniok, and D. Labate, "Sparse multidimensional representations using anisotropic dilation and shear operators," in Wavelets and Splines (G. Chen and M. J. Lai, eds.), pp. 189-201, Nashboro Press, 2006.

[28] G. Kutyniok and D. Labate, eds., Shearlets: Multiscale analysis for multivariate data. Birkhäuser, 2012.

[29]E. J. Candès, Ridgelets: Theory and applications. PhD thesis, Stanford University, 1998.

[30] E. J. Candès and D. L. Donoho, "Ridgelets: A key to higher-dimensional intermittency?," Philos. Trans. R. Soc. London Ser. A, vol. 357, no. 1760 , pp. 2495-2509, 1999.

[31] P. Grohs, "Ridgelet-type frame decompositions for Sobolev spaces related to linear transport," J. Fourier Anal. Appl., vol. 18, no. 2, pp. 309-325, 2012.

[32] S. T. Ali, J. P. Antoine, and J. P. Gazeau, "Continuous frames in Hilbert spaces," Annals of Physics, vol. 222, no. 1, pp. 1-37, 1993.

[33] O. Christensen, An introduction to frames and Riesz bases. Birkhäuser, 2003.

[34] G. Kaiser, A friendly guide to wavelets. Birkhäuser, 1994

[35] D. L. Donoho, "Sparse components of images and optimal atomic decompositions," Constructive Approximation, vol. 17, no. 3, pp. 353$382,2001$.

[36] P. Grohs and G. Kutyniok, "Parabolic molecules," Foundations of Computational Mathematics, vol. 14, no. 2, pp. 299-337, 2014.

[37] G. Kutyniok and D. Labate, "Introduction to shearlets," in Shearlets: Multiscale analysis for multivariate data [28], pp. 1-38.

[38] Y. LeCun and C. Cortes, "The MNIST database of handwritten digits." http://yann.lecun.com/exdb/mnist//. 1998

[39] B. Dacorogna, Introduction to the calculus of variations. Imperial College Press, 2004.

[40] W. Rudin, Functional analysis. McGraw-Hill, 2nd ed., 1991.

[41] L. Grafakos, Modern Fourier analysis. Springer, 2nd ed., 2009.

[42] A. R. Adams, Sobolev spaces. Academic Press, 1975.

[43] M. Frazier, B. Jawerth, and G. Weiss, Littlewood-Paley theory and the study of function spaces. American Mathematical Society, 1991.

[44] T. Wiatowski, P. Grohs, and H. Bölcskei, "Topology reduction in deep convolutional feature extraction networks," Proc. of SPIE (Wavelets and Sparsity XVII), vol. 10394, pp. 1039418:1-1039418:12, 2017.

[45] K. Gröchening, A. J. E. M. Janssen, N. Kaiblinger, and G. E. Pfander, "Note on B-Splines, wavelet scaling functions, and Gabor frames," IEEE Trans. Inf. Theory, vol. 49, no. 12, pp. 3318-3320, 2003.

[46] P. Vandergheynst, "Directional dyadic wavelet transforms: Design and algorithms," IEEE Trans. Image Process., vol. 11, no. 4, pp. 363-372, 2002.

[47] I. Waldspurger, "Exponential decay of scattering coefficients," Proc. of International Conference on Sampling Theory and Applications (SampTA), pp. 143-146, 2017. 
[48] K. Jarrett, K. Kavukcuoglu, M. Ranzato, and Y. LeCun, "What is the best multi-stage architecture for object recognition?," in Proc. of IEEE International Conference on Computer Vision (ICCV), pp. 2146-2153, 2009.

[49] M. A. Ranzato, F. J. Huang, Y. Boureau, and Y. LeCun, "Unsupervised learning of invariant feature hierarchies with applications to object recognition," in Proc. of IEEE International Conference on Computer Vision and Pattern Recognition (CVPR), pp. 1-8, 2007.

[50] H. Wendland, Scattered data approximation. Cambridge University Press, 2004.

[51] M. P. do Carmo, Riemannian geometry, 3rd ed.. Birkhäuser, 2013.

[52] T. Runst and W. Sickel, Sobolev spaces of fractional order, Nemytskij operators, and nonlinear partial differential equations, vol. 3. Walter de Gruyter, 1996.

[53] A. Gray, Tubes. Springer, 2nd ed., 2004.

[54] H. Weyl, "On the volume of tubes," Amer. J. Math., vol. 61, pp. 461-472, 1939.

[55] E. DiBenedetto, Real analysis. Birkhäuser, 2002.

Thomas Wiatowski was born in Strzelce Opolskie, Poland, on December 20, 1987, and received the BSc in Mathematics and the MSc in Mathematics from Technical University of Munich, Germany, in 2010 and 2012, respectively. In 2012 he was a researcher at the Institute of Computational Biology at the Helmholtz Zentrum in Munich, Germany. He joined ETH Zurich in 2013 where he graduated with the Dr. sc. degree in 2017. His research interests are in deep machine learning, mathematical signal processing, and applied harmonic analysis.

Philipp Grohs received the MSc and PhD degrees in Applied Mathematics from Vienna University of Technology in 2006 and 2007, respectively. He is currently an Associate Professor of Mathematics at the University of Vienna. His research interests are in signal processing, numerical analysis, computational harmonic analysis, machine learning, and computational geometry. He is the recipient of the 2014 ETH Latsis Prize. Since 2017 he has been serving as an associate editor of the IEEE Transactions on Information Theory. He is a member of the board of directors of the Austrian Mathematical Society.

Helmut Bölcskei was born in Mödling, Austria, on May 29, 1970, and received the Dipl.-Ing. and Dr. techn. degrees in electrical engineering from Vienna University of Technology, Vienna, Austria, in 1994 and 1997, respectively. In 1998 he was with Vienna University of Technology. From 1999 to 2001 he was a postdoctoral researcher in the Information Systems Laboratory, Department of Electrical Engineering, and in the Department of Statistics, Stanford University, Stanford, CA. He was in the founding team of Iospan Wireless Inc., a Silicon Valley-based startup company (acquired by Intel Corporation in 2002) specialized in multiple-input multiple-output (MIMO) wireless systems for high-speed Internet access, and was a cofounder of Celestrius AG, Zurich, Switzerland. From 2001 to 2002 he was an Assistant Professor of Electrical Engineering at the University of Illinois at Urbana-Champaign. He has been with ETH Zurich since 2002, where he is a Professor of Electrical Engineering. He was a visiting researcher at Philips Research Laboratories Eindhoven, The Netherlands, ENST Paris, France, and the Heinrich Hertz Institute Berlin, Germany. His research interests are in information theory, mathematical signal processing, machine learning, and statistics.

He received the 2001 IEEE Signal Processing Society Young Author Best Paper Award, the 2006 IEEE Communications Society Leonard G. Abraham Best Paper Award, the 2010 Vodafone Innovations Award, the ETH "Golden Owl" Teaching Award, is a Fellow of the IEEE, a 2011 EURASIP Fellow, was a Distinguished Lecturer (2013-2014) of the IEEE Information Theory Society, an Erwin Schrödinger Fellow (1999-2001) of the Austrian National Science Foundation (FWF), was included in the 2014 Thomson Reuters List of Highly Cited Researchers in Computer Science, and is the 2016 Padovani Lecturer of the IEEE Information Theory Society. He served as an associate editor of the IEEE Transactions on Information Theory, the IEEE Transactions on Signal Processing, the IEEE Transactions on Wireless Communications, and the EURASIP Journal on Applied Signal Processing. He was editor-in-chief of the IEEE Transactions on Information Theory during the period 2010-2013. He served on the editorial board of the IEEE Signal Processing Magazine and is currently on the editorial boards of "Foundations and Trends in Networking" and "Foundations and Trends in Communications and Information Theory". He was TPC co-chair of the 2008 IEEE International Symposium on Information Theory and the 2016 IEEE Information Theory Workshop and currently serves as 2nd vice president of the IEEE Information Theory Society. He has been a delegate of the president of ETH Zurich for faculty appointments since 2008 . 